\title{
Galoisian Approach to integrability of Schrödinger Equation
}

\author{
Primitivo B. Acosta-Humánez, Juan J. Morales-Ruiz, \\ and Jacques-Arthur Weil
}

Dedicated to Jerry Kovacic, in memoriam.

\begin{abstract}
In this paper, we examine the non-relativistic stationary Schrödinger equation from a differential Galois-theoretic perspective. The main algorithmic tools are pullbacks of second order ordinary linear differential operators, so as to achieve rational function coefficients ("algebrization"), and Kovacic's algorithm for solving the resulting equations. In particular, we use this Galoisian approach to analyze Darboux transformations, Crum iterations and supersymmetric quantum mechanics. We obtain the ground states, eigenvalues, eigenfunctions, eigenstates and differential Galois groups of a large class of Schrödinger equations, e.g. those with exactly solvable and shape invariant potentials (the terms are defined within). Finally, we introduce a method for determining when exact solvability is possible.
\end{abstract}

\section{Contents}

1. Introduction

2. Differential Galois Theory

3. Notions of Algebraically Solvable and Quasi-Solvable Potentials 11

4. Galoisian Aspects of Darboux Transformations and Shape Invariance 13

5. Schrödinger Equations with Rational Potentials 21

6. Transcendental Potentials and the Algebrization Method 30

Final Remarks

Acknowledgments $\quad 56$

Appendix A. Kovacic's Algorithm 5

References

1991 Mathematics Subject Classification. Primary 12H05, 81Q60; Secondary 65L80, 81T60.

Key words and phrases. Algebraic Spectrum, Darboux Transformations, Differential Galois Theory, Exactly solvable potentials, Quasi-Exactly solvable potentials, Schrödinger Equation, Shape Invariant Potentials, Supersymmetric Quantum Mechanics. 


\section{Introduction}

In classical mechanics, the Hamiltonian corresponding to the energy (kinetic plus potential) is given by

$$
H=\frac{\|\vec{p}\|^{2}}{2 m}+U(\vec{x}), \quad \vec{p}=\left(p_{1}, \ldots, p_{n}\right), \quad \vec{x}=\left(x_{1}, \ldots, x_{n}\right),
$$

and the corresponding (Hamiltonian) equations are $\dot{\vec{x}}=\frac{\partial H}{\partial \vec{p}}$ and $\dot{\vec{p}}=-\frac{\partial H}{\partial \vec{x}}$. In quantum mechanics, the momentum $\vec{p}$ is replaced by $\vec{p}=-\imath \hbar \nabla$, the Hamiltonian operator by the Schrödinger (non-relativistic, stationary) operator

$$
H=-\frac{\hbar^{2}}{2 m} \nabla^{2}+U(\vec{x})
$$

and Hamilton's equations by the Schrödinger equation $H \Psi=E \Psi$.

The eigenfunction $\Psi$ is known as the wave function, the eigenvalue $E$ is the energy level, $U(\vec{x})$ is the potential or potential energy. Solutions $(\Psi, E)$ of the Schrödinger equation are the states of the particle. In specific applications, the potentials will satisfy some conditions depending of physical restrictions such as barrier, scattering, etc., see [16, 27, 38, 42, 54.

The Schrödinger operator is known to be a self-adjoint operator: $H^{\dagger}=H$ when viewed within the context of a suitable complex and separable Hilbert space. As a consequence, the spectrum $\operatorname{Spec}(H)$ of $H$ must be real, bounded below, and be the disjoint union of the point spectrum $\operatorname{Spec}_{p}(H)$ and the continuous spectrum $\operatorname{Spec}_{c}(H)$ (see for example [5, [50, 61]). Wave functions belonging to the continuous spectrum may arise from barrier potentials and from periodic boundary conditions. The transmission and reflection coefficients are related with the barrier potentials, 27.

In this paper, we consider the one-dimensional Schrödinger equation with the normalization $\hbar=2 m=1$, i.e.

$$
H \Psi=E \Psi, \quad H=-\partial_{z}^{2}+V(z),
$$

where we may have $z=x$ (cartesian coordinate) or $z=r$ (radial coordinate). We let $\Psi_{n}$ denote the wave function corresponding to an energy level $E=E_{n}$.

Our purpose is to investigate solutions and their classification from the viewpoint of differential Galois theory. We should point out that this viewpoint has already been explored in nearby theories, for example by Spiridonov in 60. For sake of completeness, we now recall a few definitions related to quantum mechanics, and several useful results on the one-dimensional Schrödinger equation.

Bound States A solution $\Psi_{n}$ is called a bound state when its norm is finite and the corresponding eigenvalue $E_{n}$ belongs to the point spectrum of $H$, i.e.

$$
E_{n} \in \operatorname{Spec}_{p}(H), \quad \int\left|\Psi_{n}(x)\right|^{2} d x<\infty, \quad n \in \mathbb{Z}_{+} .
$$

Ground State and Excited States. Wave functions $\Psi_{0}, \Psi_{1}, \ldots, \Psi_{n}, \ldots$ of the bound states are numbered so that the corresponding energies are ordered $E_{0}<E_{1}<\cdots<E_{n}<\ldots$. The state $\left(\Psi_{0}, E_{0}\right)$ with minimal energy is called the ground state and the remaining states $\Psi_{1}, \ldots, \Psi_{n}, \ldots$ are called the excited states. 
Scattering States. A solution $\Psi$ corresponding to the level energy $E$ is called a scattering state when $E$ belongs to the continuous spectrum of $H$ and its norm is infinite.

To model a particle moving in one dimension, we use the classical one dimensional Schrödinger equation with Cartesian coordinate $x$. We could also consider motion in three dimension by reducing the associated Schrödinger equation to the one-dimensional radial equation (see [27, 38, [54]).

Darboux Transformation. The Darboux transformation is a process which, starting from a second order linear differential equation such as the Schrödinger equation, produces a family of second order differential equations with a similar shape. This vague formulation is made clear in the following theorem, taken from from 20, which is slightly more general than what is known today as the Darboux transformation.

For any value of a constant $m$, let $y=y_{m}$ denote a solution of the equation

$$
\partial_{x}^{2} y+P \partial_{x} y+(Q-m R) y=0 .
$$

Suppose, in addition, that we are given a solution $\theta$ of the equation

$$
\partial_{x}^{2} \theta+P \partial_{x} \theta+Q \theta=0 .
$$

Then the function

$$
u=\frac{\partial_{x} y-\frac{\partial_{x} \theta}{\theta} y}{\sqrt{R}}
$$

is a solution of the equation

$$
\partial_{x}^{2} u+P \partial_{x} u+\left(\theta \sqrt{R} \partial_{x}\left(\frac{P}{\theta \sqrt{R}}\right)-\theta \sqrt{R} \partial_{x}^{2}\left(\frac{1}{\theta \sqrt{R}}\right)-m R\right) u=0,
$$

when $m \neq 0$.

This general Darboux transformation $(y, \theta) \mapsto u$ preserves the shape of the differential equation and maps $Q$ to $\theta \sqrt{R} \partial_{x}\left(\frac{P}{\theta \sqrt{R}}\right)-\theta \sqrt{R} \partial_{x}^{2}\left(\frac{1}{\theta \sqrt{R}}\right)$. In [20, 21], Darboux considered the particular case $R=1$ and $P=0$, which is known today as the Darboux transformation. It is the following corollary of the above:

For any value of a constant $m$, let $y=y_{m}$ denote a solution of the equation

$$
\partial_{x}^{2} y=(f(x)+m) y .
$$

Suppose, in addition, that we are given a solution $\theta$ of the equation $\partial_{x}^{2} \theta=(f(x)+$ $\left.m_{1}\right) \theta$ for some fixed value $m_{1}\left(e . g m_{1}=0\right)$. Then the function

$$
u=\partial_{x} y-\frac{\partial_{x} \theta}{\theta} y
$$

is a solution of the equation

$$
\partial_{x}^{2} u=\left(\theta \partial_{x}^{2}\left(\frac{1}{\theta}\right)-m_{1}+m\right) u,
$$

for $m \neq m_{1}$. Furthermore,

$$
\theta \partial_{x}^{2}\left(\frac{1}{\theta}\right)-m_{1}=f(x)-2 \partial_{x}\left(\frac{\partial_{x} \theta}{\theta}\right)=2\left(\frac{\partial_{x} \theta}{\theta}\right)^{2}-f(x)-2 m_{1} .
$$


Some examples can be found as exercises in the book of Ince [30 p. 132]. Darboux's transformation is related with with Delsarte's transformation operators (named transmutations in [22]), which today are called intertwiners or intertwining operators. A good short survey about Darboux transformations can be found in [52.

Crum, inspired by the works of Liouville 39, 40 obtained an iterative generalization of Darboux's result appropriate for Sturm-Liouville systems: he proved that the Sturm-Liouville conditions are preserved under Darboux transformations, see 19. Crum's result is presented in the following theorem. Recall that the Wronskian determinant $W r$ of $k$ functions $f_{1}, f_{2}, \ldots, f_{k}$ is

$$
W r\left(f_{1}, \ldots, f_{k}\right)=\operatorname{det} A, \quad A_{i j}=\partial_{x}^{i-1} f_{j}, \quad i, j=1,2, \ldots, k .
$$

Crum's Theorem. Let $\Psi_{1}, \Psi_{2}, \ldots \Psi_{n}$ be solutions of the Schrödinger equation $H \Psi=E \Psi$ for energy levels $E=E_{1}, E_{2}, \ldots, E_{n}$ respectively. Letting

$$
\Psi[n]=\frac{W r\left(\Psi_{1}, \ldots, \Psi_{n}, \Psi\right)}{W r\left(\Psi_{1}, \ldots, \Psi_{n}\right)}, \quad \text { and } V[n]=V-2 \partial_{x}^{2} \ln W r\left(\Psi_{1}, \ldots, \Psi_{n}\right),
$$

we obtain the Schrödinger equation

$$
H^{[n]} \Psi[n]=E \Psi[n], \quad \text { where } H^{[n]}=-\partial_{x}^{2}+V[n] \quad \text { for } E \neq E_{i}, 1 \leq i \leq n
$$

Crum's transformation coincides with Darboux's transformation when $n=1$; as shown in 47, iterations of Darboux transformations coincide with the Crum iteration. Both formalisms allow us to obtain new families of Schrödinger equations while preserving the spectrum and the Sturm-Liouville conditions, see 42, 47. We note that there are extensions of Crum's iteration connecting the Sturm-Liouville theory with orthogonal polynomial theory $\mathbf{3 7}$.

Supersymmetric quantum mechanical system. Witten introduced, in [72, §6], some models for which dynamical breaking of supersymmetry is possible.

A supersymmetric quantum mechanical system is described by a Hamiltonian $\mathcal{H}$ together with several (self-adjoint) operators $Q_{i}$ which commute with the hamiltonian $\left(\left[Q_{i}, \mathcal{H}\right]=0\right)$ and satisfy the anticommutation relations

$$
\left\{Q_{i}, Q_{j}\right\}=\delta_{i j} \mathcal{H}, \quad \text { where }\left\{Q_{i}, Q_{j}\right\}=Q_{i} Q_{j}+Q_{j} Q_{i} .
$$

The simplest example of a supersymmetric quantum mechanical system arises in the case $n=2$, which is considered in this paper. The wave function of $\mathcal{H} \Phi=E \Phi$ is then a two-component Pauli spinor,

$$
\Phi(x)=\left(\begin{array}{l}
\Psi_{+}(x) \\
\Psi_{-}(x)
\end{array}\right) .
$$

The supercharges $Q_{i}$ are defined as

$$
Q_{ \pm}=\frac{\sigma_{1} p \pm \sigma_{2} W(x)}{2}, \quad Q_{+}=Q_{1}, Q_{-}=Q_{2}, \quad p=-i \partial_{x},
$$

where the superpotential $W$ is an arbitrary function of $x$ and $\sigma_{i}$ are the usual Pauli spin matrices. Using the expressions above we obtain $\mathcal{H}$ :

$$
\mathcal{H}=2 Q_{-}^{2}=2 Q_{+}^{2}=\frac{I_{2} p^{2}+I_{2} W^{2}(x)+\sigma_{3} \partial_{x} W(x)}{2}, \quad \text { with } I_{2}=\left(\begin{array}{ll}
1 & 0 \\
0 & 1
\end{array}\right) .
$$


The supersymmetric partner Hamiltonians $H_{ \pm}$are given by

$$
H_{ \pm}=-\frac{1}{2} \partial_{x}^{2}+V_{ \pm}, \quad V_{ \pm}=\left(\frac{W}{\sqrt{2}}\right)^{2} \pm \frac{1}{\sqrt{2}} \partial_{x}\left(\frac{W}{\sqrt{2}}\right) .
$$

The potentials $V_{ \pm}$are called supersymmetric partner potentials and are linked with the superpotential $W$ through a Riccati equation. Then $\mathcal{H}$ can be written as

$$
\mathcal{H}=\left(\begin{array}{cc}
H_{+} & 0 \\
0 & H_{-}
\end{array}\right)
$$

which leads us to the Schrödinger equations $H_{+} \Psi_{+}=E \Psi_{+}$and $H_{-} \Psi_{-}=E \Psi_{-}$. To solve $\mathcal{H} \Phi=E \Phi$ is equivalent to solve simultaneously $H_{+} \Psi_{+}=E \Psi_{+}$and $H_{-} \Psi_{-}=E \Psi_{-}$.

In [72, §6], V.B. Matveev and M. Salle recast Darboux transformations in this context; they interpret the Darboux theorem as a Darboux covariance of a SturmLiouville problem and prove the following result (see also [52, §5-6]).

The case $n=2$ in Supersymetric Quantum Mechanics is equivalent to a single Darboux transformation.

As a generalization of the method to solve the harmonic oscillator [28, 23, the ladder (raising and lowering) operators are defined as

$$
A^{+}=-\partial_{x}-\frac{\partial_{x} \Psi_{0}}{\Psi_{0}}, \quad A=\partial_{x}-\frac{\partial_{x} \Psi_{0}}{\Psi_{0}}
$$

which are strongly related with the supercharges $Q_{ \pm}$in Witten's formalism. We have,

$$
\begin{gathered}
A \Psi_{0}=0, \quad A^{+} A=H_{-}, \quad A A^{+}=H_{+}=-\partial_{x}^{2}+V_{+}(x), \quad \text { where } \\
V_{+}(x)=V_{-}(x)-2 \partial_{x}\left(\frac{\partial_{x} \Psi_{0}}{\Psi_{0}}\right)=-V_{-}(x)+2\left(\frac{\partial_{x} \Psi_{0}}{\Psi_{0}}\right)^{2} .
\end{gathered}
$$

The supersymmetric partner potentials $V_{+}$and $V_{-}$have the same energy levels, except for $E_{0}^{(-)}=0$. In terms of the superpotential $W(x)$, the operators $A$ and $A^{+}$ are given by

$$
A^{+}=-\partial_{x}+W(x), \quad A=\partial_{x}+W(x) .
$$

Similarly, the supersymmetric partner potentials $V_{ \pm}(x)$ and the superpotential $W(x)$ satisfy:

$$
\frac{V_{+}(x)+V_{-}(x)}{2}=W^{2}(x), \quad\left[A, A^{+}\right]=2 \partial_{x} W(x) .
$$

Shape invariance. Gendenshtein, in his remarkable paper 28, introduced the concept of shape invariance, which is a property of families of potentials with respect to their parameters. Given a family $a=\left(a_{n}\right)_{n}$ of parameters, the shape invariance condition can be expressed as :

$$
V_{n+1}\left(x ; a_{n}\right)=V_{n}\left(x ; a_{n+1}\right)+R\left(a_{n}\right), \quad V_{-}=V_{0}, \quad V_{+}=V_{1},
$$

where $R$ is a remainder, which does not depends on $x$. When this relation holds, we say that $V=V_{-}=V_{0}$ is a shape invariant potential (with respect to the given family or transformation), see also [52, 16, 23. 
Gendenshteïn Theorem. Consider the Schrödinger equation $H \Psi=E_{n} \Psi$, where $V=V_{-}=V_{0}$ is a shape invariant potential. If we fix the first level of energy $E_{0}=0$, then the excited spectrum and the wave functions are given respectively by

$$
E_{n}=\sum_{k=2}^{n+1} R\left(a_{k}\right), \quad \Psi_{n}^{(-)}\left(x ; a_{1}\right)=\prod_{k=1}^{n} A^{+}\left(x ; a_{k}\right) \Psi_{0}^{(-)}\left(x ; a_{n+1}\right) .
$$

The structure of the rest of the paper is as follows. In section 2 we introduce the necessary concepts and results of the Differential Galois Theory of linear ordinary differential equations. In section 3 we introduce some terminology about solvability and we obtain some results about the invariance of the Galois group with respect to Darboux transformations. Section 4 is devoted to the study of the rational potentials and section 5 to transcendental potentials by means of the algebrization method.

\section{Differential Galois Theory}

In this section, we recall some differential Galois material to study the onedimensional Schrödinger equation,

$$
\partial_{x}^{2} \Psi=(V-\lambda) \Psi
$$

where $V$ is some function and $\lambda$ is a constant (the spectral parameter). There are many references on differential Galois theory (or "Picard-Vessiot" theory), for example [11, 18, 43, 65.

2.1. Picard-Vessiot Galois theory. We establish an algebraic model for functions and the corresponding Galois theory.

Differential Fields. Let $K$ be a commutative field of characteristic zero. A derivation of $K$ is a map $\partial_{x}: K \rightarrow K$ satisfying $\partial_{x}(a+b)=\partial_{x} a+\partial_{x} b$ and $\partial_{x}(a b)=\partial_{x} a \cdot b+a \cdot \partial_{x} b$ for all $a, b \in K$. We then say that $\left(K, \partial_{x}\right)$ (or just $K$, when there is no ambiguity) is a differential field with the derivation $\partial_{x}$.

We assume that $K$ contains an element $x$ such that $\partial_{x}(x)=1$. Let $\mathcal{C}$ denote the field of constants of $K$ :

$$
\mathcal{C}=\left\{c \in K \mid \partial_{x} c=0\right\} .
$$

It is also of characteristic zero and will be assumed to be algebraically closed.

Throughout this paper, the coefficient field for a differential equation (e.g the Schrödinger equation $\partial_{x}^{2} \Psi=(V-\lambda) \Psi$ ) is defined as the smallest differential field containing all the coefficients of the equation.

We will mostly analyze second order linear homogeneous differential equations, i.e equations of the form

$$
\mathcal{L}:=\partial_{x}^{2} y+a \partial_{x} y+b y=0, \quad a, b \in K .
$$

so the rest of the theory will be explained in this context.

Picard-Vessiot Extension. Let $L$ be a differential field containing $K$ (a differential extension of $K$ ). We say that $L$ is a Picard-Vessiot extension of $K$ for $\mathcal{L}$ if there exist two linearly independent $y_{1}, y_{2} \in L$ solutions of $\mathcal{L}$ such that $L=K\left\langle y_{1}, y_{2}\right\rangle$ (i.e $L=K\left(y_{1}, y_{2}, \partial_{x} y_{1}, \partial_{x} y_{2}\right)$ ) and $L$ and $K$ has the same field of 
constants $\mathcal{C}$.

In what follows, we choose a Picard-Vessiot extension and the term "solution of $\mathcal{L}$ " will mean "solution of $\mathcal{L}$ in $L$ ". So any solution of $\mathcal{L}$ is a linear combination (over $\mathcal{C}$ ) of $y_{1}$ and $y_{2}$.

Differential Galois Groups A $K$-automorphism $\sigma$ of the Picard-Vessiot extension $L$ is called a differential automorphism if it leaves $K$ fixed and commutes with the derivation. This means that $\sigma\left(\partial_{x} a\right)=\partial_{x}(\sigma(a))$ for all $a \in L$ and $\forall a \in K$, $\sigma(a)=a$.

The group of all differential automorphisms of $L$ over $K$ is called the differential Galois group of $L$ over $K$ and is denoted by $\operatorname{DGal}(L / K)$.

Given $\sigma \in \operatorname{DGal}(L / K)$, we see that $\left\{\sigma y_{1}, \sigma y_{2}\right\}$ are also solutions of $\mathcal{L}$. Hence there exists a matrix

$$
A_{\sigma}=\left(\begin{array}{ll}
a & b \\
c & d
\end{array}\right) \in \mathrm{GL}(2, \mathbb{C})
$$

such that

$$
\sigma\left(\left(\begin{array}{ll}
y_{1} & y_{2}
\end{array}\right)\right)=\left(\begin{array}{ll}
\sigma\left(y_{1}\right) & \sigma\left(y_{2}\right)
\end{array}\right)=\left(\begin{array}{ll}
y_{1} & y_{2}
\end{array}\right) A_{\sigma} .
$$

As $\sigma$ commutes with the derivation, this extends naturally to an action on a fundamental solution matrix of the companion first order system associated with $\mathcal{L}$.

$$
\sigma\left(\left(\begin{array}{cc}
y_{1} & y_{2} \\
\partial_{x} y_{1} & \partial_{x} y_{2}
\end{array}\right)\right)=\left(\begin{array}{cc}
\sigma\left(y_{1}\right) & \sigma\left(y_{2}\right) \\
\sigma\left(\partial_{x} y_{1}\right) & \sigma\left(\partial_{x} y_{2}\right)
\end{array}\right)=\left(\begin{array}{cc}
y_{1} & y_{2} \\
\partial_{x} y_{1} & \partial_{x} y_{2}
\end{array}\right) A_{\sigma} .
$$

This defines a faithful representation $\operatorname{DGal}(L / K) \rightarrow \mathrm{GL}(2, \mathbb{C})$ and it is possible to consider $\operatorname{DGal}(L / K)$ as a subgroup of $\mathrm{GL}(2, \mathbb{C})$. It depends on the choice of the fundamental system $\left\{y_{1}, y_{2}\right\}$, but only up to conjugacy.

Recall that an algebraic group $G$ is an algebraic manifold endowed with a group structure. Let $\mathrm{GL}(n, \mathbb{C})$ denote, as usual, the set of invertible $n \times n$ matrices with entries in $\mathbb{C}$ (and $\operatorname{SL}(n, \mathbb{C})$ be the set of matrices with determinant equal to 1 ). A linear algebraic group will be a subgroup of $\mathrm{GL}(n, \mathbb{C})$ equipped with a structure of algebraic group. One of the fundamental results of the Picard-Vessiot theory is the following theorem (see [33, 35]).

The differential Galois group $\mathrm{DGal}(L / K)$ is an algebraic subgroup of $\mathrm{GL}(2, \mathbb{C})$. In fact, the differential Galois group measures the algebraic relations between the solutions (and their derivatives). It is sometimes viewed as the object which should tell "what algebra sees of the dynamics of the solutions".

In an algebraic group $G$, the largest connected algebraic subgroup of $G$ containing the identity, noted $G^{\circ}$, is a normal subgroup of finite index. It is often called the connected component of the identity. If $G=G^{0}$ then $G$ is a connected group.

When $G^{0}$ satisfies some property, we say that $G$ virtually satisfies this property. For example, virtually solvability of $G$ means solvability of $G^{0}$ and virtual abelianity of 
$G$ means abelianity of $G^{0}$ (see [71).

Lie-Kolchin Theorem. Let $G \subseteq \mathrm{GL}(2, \mathbb{C})$ be a virtually solvable group. Then $G^{0}$ is triangularizable, i.e it is conjugate to a subgroup of upper triangular matrices.

2.2. Algebraic Subgroups of $S L(2, \mathbb{C})$. We recall below some examples of subgroups of $\operatorname{SL}(2, \mathbb{C})$ for later use.

Reducible subgroups These are the groups which leave a non-trivial subspace of $V$ invariant. They are classified in two categories.

Diagonal groups: the identity group: $\{e\}=\left\{\left(\begin{array}{ll}1 & 0 \\ 0 & 1\end{array}\right)\right\}$, the $n$-roots: $\mathbb{G}^{[n]}=$ $\left\{\left(\begin{array}{cc}c & 0 \\ 0 & c^{-1}\end{array}\right), \quad c^{n}=1\right\}$, the multiplicative group: $\mathbb{G}_{m}=\left\{\left(\begin{array}{cc}c & 0 \\ 0 & c^{-1}\end{array}\right), \quad c \in \mathbb{C}^{*}\right\}$ Triangular groups: the additive group: $\mathbb{G}_{a}=\left\{\left(\begin{array}{ll}1 & d \\ 0 & 1\end{array}\right), \quad d \in \mathbb{C}\right\}$, the $n$-quasiroots: $\mathbb{G}^{\{n\}}=\left\{\left(\begin{array}{cc}c & d \\ 0 & c^{-1}\end{array}\right), \quad c^{n}=1, \quad d \in \mathbb{C}\right\}$, the Borel group: $\mathbb{B}=\mathbb{C}^{*} \ltimes \mathbb{C}=$ $\left\{\left(\begin{array}{cc}c & d \\ 0 & c^{-1}\end{array}\right), \quad c \in \mathbb{C}^{*}, \quad d \in \mathbb{C}\right\}$

Irreducible subgroups The infinite dihedral group (also called meta-abelian group):

$\mathbb{D}_{\infty}=\left\{\left(\begin{array}{cc}c & 0 \\ 0 & c^{-1}\end{array}\right), \quad c \in \mathbb{C}^{*}\right\} \cup\left\{\left(\begin{array}{cc}0 & d \\ -d^{-1} & 0\end{array}\right), \quad d \in \mathbb{C}^{*}\right\}$ and its finite subgroups $\mathbb{D}_{2 n}$ (where $c$ and $d$ spans the $n$-th roots of unity).

There are also three other finite irreducible (primitive) groups: the tetrahedral group $A_{4}^{\mathrm{SL}_{2}}$ of order 24 , the octahedral group $S_{4}^{\mathrm{SL}_{2}}$ of order 48 , and the icosahedral group $A_{5}^{\mathrm{SL}_{2}}$ of order 120 .

Integrability. We say that the linear differential equation $\mathcal{L}$ is (Liouville) integrable if the Picard-Vessiot extension $L \supset K$ is obtained as a tower of differential fields $K=L_{0} \subset L_{1} \subset \cdots \subset L_{m}=L$ such that $L_{i}=L_{i-1}(\eta)$ for $i=1, \ldots, m$, where either

(1) $\eta$ is algebraic over $L_{i-1}$, that is $\eta$ satisfies a polynomial equation with coefficients in $L_{i-1}$.

(2) $\eta$ is primitive over $L_{i-1}$, that is $\partial_{x} \eta \in L_{i-1}$.

(3) $\eta$ is exponential over $L_{i-1}$, that is $\partial_{x} \eta / \eta \in L_{i-1}$.

We remark that the usual terminology in differential algebra for integrable equations is that the corresponding Picard-Vessiot extensions are called Liouvillian. The following theorem is due to Kolchin.

The equation $\mathcal{L}$ is integrable if and only if $\mathrm{DGal}(L / K)$ is virtually solvable.

2.3. Eigenrings. Eigenrings are a tool, introduced by M.F. Singer, to study the decomposability of systems or operators. A general reference is [6] (and references therein) . 
Eigenrings of Systems. Consider the system $\partial_{x} X=-A X$, denoted by $[A]$, where $A \in \mathrm{GL}(2, K)$. The eigenring of the system $[A]$, denoted by $\mathcal{E}(A)$, is the set of $2 \times 2$ matrices $P$ in $K$ satisfying

$$
\partial_{x} P=P A-A P .
$$

Eigenrings of Operators. Let $\mathfrak{L}$ be a second order differential operator, i.e $\mathcal{L}:=\mathfrak{L}(y)=0$. Denote $V(\mathfrak{L})$ as the solution space of $\mathcal{L}$. The eigenring of $\mathfrak{L}$, denoted by $\mathcal{E}(\mathfrak{L})$, is the set of all operators $\mathfrak{R}$ of order less than that of $\mathfrak{L}$ for which $\mathfrak{R}(V(\mathfrak{L}))$ is a subset of $V(\mathfrak{L})$, that is $\mathfrak{L} \mathfrak{R}=\mathfrak{S} \mathfrak{L}$, where $\mathfrak{S}$ is also an operator. For more details see [59, 6, 66, 67, 68.

We study an equation $\mathfrak{L}(y)=0$ with $\mathfrak{L}=\partial_{x}^{2}+p \partial_{x}+q$; this is equivalent to the system $[A]$, where $A$ is given by

$$
A=\left(\begin{array}{cc}
0 & -1 \\
q & p
\end{array}\right) \quad p, q \in K .
$$

Relations between the system approach and the operator approach are given in the following simple lemmas .

Lemma. Let $\mathfrak{L}=\partial_{x}^{2}+p \partial_{x}+q, A=\left(\begin{array}{cc}0 & -1 \\ q & p\end{array}\right)$ and $P=\left(\begin{array}{ll}a & b \\ c & d\end{array}\right)$. Then:

(1) If $P \in \mathcal{E}(A)$, then $\mathfrak{R}=a+b \partial_{x} \in \mathcal{E}(\mathfrak{L})$.

(2) If $\mathfrak{R}=a+b \partial_{x} \in \mathcal{E}(\mathfrak{L})$, then $P \in \mathcal{E}(A)$, where $P$ is given by

$$
P=\left(\begin{array}{cc}
a & b \\
\partial_{x} a-b q & a+\partial_{x} b-b p
\end{array}\right) .
$$

(3) $1 \leq \operatorname{dim}_{\mathcal{C}} \mathcal{E}(\mathfrak{L}) \leq 4$.

(4) $P \in \mathrm{GL}(2, K) \Leftrightarrow \frac{\partial_{x} a}{a}-\frac{a}{b}+p \neq \frac{\partial_{x} b}{b}-\frac{b}{a} q$.

Lemma. Assume that $\mathfrak{L}=\partial_{x}^{2}+b$, where $b \in K$. The following statements hold:

(1) If $\operatorname{dim}_{\mathcal{C}} \mathcal{E}(\mathfrak{L})=1$, then either the differential Galois group is irreducible $\left(\mathbb{D}_{\infty}\right.$ or primitive), or indecomposable ( $G \subseteq \mathbb{B}, G$ non-diagonal).

(2) If $\operatorname{dim}_{\mathcal{C}} \mathcal{E}(\mathfrak{L})=2$, then either the differential Galois group is the additive group or it is contained in the multiplicative group (but is not the identity group).

(3) If $\operatorname{dim}_{\mathcal{C}} \mathcal{E}(\mathfrak{L})=4$, then the differential Galois group is the identity group. In this case we have 2 independent solutions $\zeta_{1}$ and $\zeta_{2}$ in which $\zeta_{1}^{2}, \zeta_{2}^{2}$ and $\zeta_{1} \zeta_{2}$ are elements of the differential field $K$, i.e. the solutions of $\mathcal{L}^{S}{ }_{2}$ belong to to the differential field $K$.

2.4. Some useful special functions. Some standard special functions will be used below.

The Gauss hypergeometric equation. It is a particular case of Riemann's equation (see [32, 48, 34, 41, 24) and is given by

$$
\partial_{x}^{2} y+\frac{(\gamma-(\kappa+\beta+1) x)}{x(1-x)} \partial_{x} y-\frac{\kappa \beta}{x(1-x)} y=0 .
$$

The cases when it is solvable can be found in the Kimura table [34] (or via Kovacic's algorithm, see appendix). 
The confluent hypergeometric equations (Kummer, Whittaker). It is a degenerate form of the hypergeometric differential equation where two of the three regular singularities merge into an irregular singularity. For example, making "1 tend to $\infty$ " in a suitable way, the hypergeometric equation may transform to two classical forms:

- Kummer's form

$$
\partial_{x}^{2} y+\frac{c-x}{x} \partial_{x} y-\frac{a}{x} y=0
$$

- Whittaker's form

$$
\partial_{x}^{2} y=\left(\frac{1}{4}-\frac{\kappa}{x}+\frac{4 \mu^{2}-1}{4 x^{2}}\right) y
$$

where the parameters of the two equations are linked by $\kappa=\frac{c}{2}-a$ and $\mu=\frac{c}{2}-\frac{1}{2}$. The Galoisian structure of these equations has been deeply studied in [41, 24]. The following theorem is due to Martinet \& Ramis, 41.

The Whittaker's differential equation is integrable if and only if either, $\kappa+\mu \in$ $\frac{1}{2}+\mathbb{N}$, or $\kappa-\mu \in \frac{1}{2}+\mathbb{N}$, or $-\kappa+\mu \in \frac{1}{2}+\mathbb{N}$, or $-\kappa-\mu \in \frac{1}{2}+\mathbb{N}$.

The Bessel equation. It is a particular case of the confluent hypergeometric equation and is given by

$$
\partial_{x}^{2} y+\frac{1}{x} \partial_{x} y+\frac{x^{2}-n^{2}}{x^{2}} y=0 .
$$

Under a suitable transformation, the reduced form of the Bessel's equation is a particular case of the Whittaker's equation. Thus, we can obtain the following well known result, see [35, p. 417] and see also [36, 43.

The Bessel's differential equation is integrable if and only if $n \in \frac{1}{2}+\mathbb{Z}$.

By double confluence of the hypergeometric equation, that is making " 0 and 1 tend to $\infty$ " in a suitable way, one gets the parabolic cylinder equation (also known as Weber's equation):

$$
\partial_{x}^{2} y=\left(\frac{1}{4} x^{2}-\frac{1}{2}-n\right) y,
$$

which is integrable if and only if $n \in \mathbb{Z}$, see $[\mathbf{3 6}, \mathbf{2 4}$. Under suitable transformations one can gets the Rehm's form of the Weber's equation:

$$
\partial_{x}^{2} y=\left(a x^{2}+2 b x+c\right) y, \quad a \neq 0,
$$

so that $\frac{b^{2}-c}{a}$ is an odd integer.

The hypergeometric equation, including confluences, is a particular case of the differential equation

$$
\partial_{x}^{2} y+\frac{L}{Q} \partial_{x} y+\frac{\lambda}{Q} y, \quad \lambda \in \mathbb{C}, \quad L=a_{0}+a_{1} x, \quad Q=b_{0}+b_{1} x+b_{2} x^{2} .
$$

We mention this because the classical orthogonal polynomials and Bessel polynomials are solutions of such an equation (see $1 \mathbf{1 5}, \mathbf{3 1}, 46$ ). We remark that integrability 
conditions and solutions of differential equations with solutions orthogonal polynomials, including Bessel polynomials, can be obtained applying Kovacic's algorithm. Similarly, one can apply Kovacic's algorithm to obtain the same results given by Kimura [34 and Martinet \& Ramis [41. Also we recall that Duval \& LodayRichaud applied Kovacic's algorithm in 24 to some families of special functions, including several of the above.

\section{Notions of Algebraically Solvable and Quasi-Solvable Potentials}

The main object of our Galoisian analysis is the one-dimensional Schrödinger equation, written as

$$
\mathcal{L}_{\lambda}:=H \Psi=\lambda \Psi, \quad H=-\partial_{x}^{2}+V(x), \quad V \in K,
$$

where $K$ is a differential field (with $\mathbb{C}$ as field of constants). We are interested in the integrability (in Galoisian sense) of equation (3.1). Denote by $L_{\lambda}$ the Picard-Vessiot extension of $K$ for $\mathcal{L}_{\lambda}$. The differential Galois group of $\mathcal{L}_{\lambda}$ is $\operatorname{DGal}\left(L_{\lambda} / K\right)$.

3.1. Algebraic Spectrum and Algebraic Solvability. Let $\Lambda \subseteq \mathbb{C}$ denote the set of eigenvalues $\lambda$ such that equation (3.1) is integrable in Galoisian sense. The set $\Lambda$ will be called the algebraic spectrum (or alternatively the Liouvillian spectral set) of $H$. We remark that $\Lambda$ can be $\emptyset$, i.e., $\operatorname{DGal}\left(L_{\lambda} / K\right)=\operatorname{SL}(2, \mathbb{C}) \forall \lambda \in \mathbb{C}$. On the other hand, the classification of subgroups of $S L_{2}(\mathbb{C})$ shows that, if $\lambda_{0} \in \Lambda$, then $\left(\operatorname{DGal}\left(L_{\lambda_{0}} / K\right)\right)^{0} \subseteq \mathbb{B}$.

We let $\Lambda_{+}$be the set $\{\lambda \in \Lambda \cap \mathbb{R}: \lambda \geq 0\}$ and $\Lambda_{-}$be the set $\{\lambda \in \Lambda \cap \mathbb{R}: \lambda \leq 0\}$.

Definition 3.1 (Algebraically Solvable and Quasi-Solvable Potentials). We say that the potential $V(x) \in K$ is:

- an algebraically solvable potential when $\Lambda$ is an infinite set, or

- an algebraically quasi-solvable potential when $\Lambda$ is a non-empty finite set, or

- an algebraically non-solvable potential when $\Lambda=\emptyset$.

When $\operatorname{Card}(\Lambda)=1$, we say that $V(x) \in K$ is a trivial algebraically quasi-solvable potential.

Examples. Let $K=\mathbb{C}(x)$.

(1) If $V(x)=x$ (Airy equation), then $\Lambda=\emptyset, V(x)$ is algebraically nonsolvable, see [33, 36.

(2) If $V(x)=0$, then $\Lambda=\mathbb{C}$, i.e., $V(x)$ is algebraically solvable. Furthermore,

$$
\operatorname{DGal}\left(L_{0} / K\right)=\{e\}, \quad \text { and } \operatorname{DGal}\left(L_{\lambda} / K\right)=\mathbb{G}_{m} \text { for } \lambda \neq 0 \text {. }
$$

(3) If $V(x)=\frac{x^{2}}{4}+\frac{1}{2}$, then $\Lambda=\{n: n \in \mathbb{Z}\}, V(x)$ is algebraically solvable. This example corresponds to the Weber's equation given previously.

(4) If $V(x)=x^{4}-2 x$, then $\Lambda=\{0\}$ and $V(x)$ is a trivial algebraically quasisolvable potential (see next section for tools to prove this).

We are interested in the spectrum (analytic spectrum) of the algebraically solvable and quasi-solvable potentials, that is, $\operatorname{Spec}(H) \cap \Lambda \neq \emptyset$. 
Potentials for which $\operatorname{Spec}(H) \cap \Lambda$ is an infinite set are called solvable (or exactly solvable) potentials (see Natanzon [45]) in the physics litterature. Similarly, potentials for which $\operatorname{Spec}(H) \cap \Lambda$ is a finite set are usually called quasi-exactly solvable (or quasi-solvable) potentials (Turbiner [63, Bender \& Dunne [8, Bender \& Boettcher [7, Saad et al. [53, Gibbons \& Vesselov [29]).

3.2. Changing the Base Field. In what follows, we will make transformations which may change the base field; typically, when $K$ is not $\mathbb{C}(x)$, we will look for transformations to another differential with rational coefficients. We now collect facts on how this affects the differential Galois group and its solvability.

Definition 3.2. Let $\mathcal{L}$ and $\widetilde{\mathcal{L}}$ be a pair of linear differential equations defined over differential fields $K$ and $\widetilde{K}$ respectively, with Picard-Vessiot extensions $L$ and $\widetilde{L}$. Let $\varphi$ be a transformation mapping $K$ to $\widetilde{K}$ and $\mathcal{L}$ to $\widetilde{\mathcal{L}}$ (so we may also choose $\varphi$ to map $L$ to $\widetilde{L}$ ). We say that:

(1) $\varphi$ is an isogaloisian transformation if

$$
\operatorname{DGal}(L / K)=\operatorname{DGal}(\widetilde{L} / \widetilde{K}) .
$$

When $\widetilde{L}=L$ and $\widetilde{K}=K$, we'll say that $\varphi$ is a strong isogaloisian transformation.

(2) $\varphi$ is a virtually isogaloisian transformation if

$$
(\operatorname{DGal}(L / K))^{0}=(\operatorname{DGal}(\widetilde{L} / \widetilde{K}))^{0} .
$$

Remark 3.3. The eigenring s of two operators $\mathfrak{L}$ and $\widetilde{\mathfrak{L}}$ are preserved under isogaloisian transformations.

Proposition 3.4. Consider the differential equations

$$
\mathcal{L}:=\partial_{x}^{2} y+a \partial_{x} y+b y=0, \quad \widetilde{\mathcal{L}}:=\partial_{x}^{2} \zeta=r \zeta, \quad a, b, r \in K .
$$

Let $\kappa \in \mathbb{Q}, f \in K, a=2 \kappa \partial_{x}(\ln f)$ and $\varphi$ be the transformation such that $\mathcal{L} \mapsto \widetilde{\mathcal{L}}$. The following statements holds:

(1) $\varphi$ is a strong isogaloisian transformation for $\kappa \in \mathbb{Z}$.

(2) $\varphi$ is a virtually strong isogaloisian transformation for $\kappa \in \mathbb{Q} \backslash \mathbb{Z}$.

Proof. Let $\mathcal{B}=\left\{y_{1}, y_{2}\right\}$ be a basis of solutions of $\mathcal{L}$ and $L$ be the corresponding Picard-Vessiot extension of $K$; similarly, let $\mathcal{B}^{\prime}=\left\{\zeta_{1}, \zeta_{2}\right\}$ be a basis of solutions of $\widetilde{\mathcal{L}}$ if a Picard-Vessiot extension $\widetilde{L}$. With the change of dependent variable $y=\zeta e^{-\frac{1}{2} \int a}$ we obtain $r=a^{2} / 4+\partial_{x} a / 2-b$ and $\widetilde{K}=K$. Thus, the relationship between $L$ and $\widetilde{L}$ depends on $a$ :

(1) If $\kappa=n \in \mathbb{Z}$, then $\mathcal{B}^{\prime}=\left\{f^{n} y_{1}, f^{n} y_{2}\right\}$ which means that $L=\widetilde{L}$ and $\varphi$ is strong isogaloisian.

(2) If $\kappa=\frac{n}{m}$, with $\operatorname{gcd}(n, m)=1, \frac{n}{m} \notin \mathbb{Z}$, then $\mathcal{B}^{\prime}=\left\{f^{\frac{n}{m}} y_{1}, f^{\frac{n}{m}} y_{2}\right\}$ which means that $\widetilde{L}$ is either an algebraic extension of degree at most $m$ of $L$, and $\varphi$ is virtually strong isogaloisian, or $L=\widetilde{L}$ when $f^{\frac{n}{m}} \in K$ which means that $\varphi$ is strong isogaloisian. 
Remark 3.5. The transformation $\varphi$ in proposition 3.4 is not injective, there are a lot of differential equations $\mathcal{L}$ that are transformed in the same differential equation $\widetilde{\mathcal{L}}$. They are called projectively equivalent.

The following is an easy consequence of the proposition:

Corollary 3.6. Let $\mathcal{L}$ be the differential equation

$$
\partial_{x}\left(a \partial_{x} y\right)=(\lambda b-\mu) y, \quad a, b \in K, \quad \lambda, \mu \in \mathbb{C}
$$

where $L, \widetilde{L}, \widetilde{\mathcal{L}}$ and $\varphi$ are given as in proposition 3.4. Then either $\widetilde{L}$ is a quadratic extension of $L$ (which means that $\varphi$ is virtually strong isogaloisian) or $\widetilde{L}=L$ (this is when $a^{\frac{1}{2}} \in K$ ) which means that $\varphi$ is strong isogaloisian.

\section{Galoisian Aspects of Darboux Transformations and Shape Invariance}

Here we present a Galoisian approach to the Darboux transformation, the Crum iteration and shape invariant potentials.

Recall that $\operatorname{Wr}\left(y_{1}, \ldots, y_{n}\right)$ denotes the Wronskian

$$
W r\left(y_{1}, \ldots, y_{n}\right)=\left|\begin{array}{ccc}
y_{1} & \cdots & y_{n} \\
\vdots & & \vdots \\
\partial_{x}^{n-1} y_{1} & \cdots & \partial_{x}^{n-1} y_{n}
\end{array}\right| .
$$

The Darboux transformation will be abreviated by DT and its $n$-th iteration by $\mathrm{DT}_{n}$; similarly, the abreviation $\mathrm{CI}_{n}$ will refer to the Crum iteration.

As above, $K$ denotes the coefficient field of the studied linear differential equation, i.e. the smallest differential containing the coefficients of the equation. We mostly consider equations over the base field $K=\mathbb{C}(x)$.

4.1. Darboux Transformations. The following result comes from the original Darboux transformation (see [20, 21), but rewritten in a way which is adapted to our Galoisian analysis.

Theorem 4.1 (Darboux Transformation). Consider the Schrödinger operators $H_{ \pm}=\partial_{x}^{2}+V_{ \pm}(x)$, with $V_{ \pm}(x) \in K$, and assume that the spectrum satisfies $\Lambda \neq \emptyset$. Let $\mathcal{L}_{\lambda}$ (resp. $\widetilde{\mathcal{L}}_{\lambda}$ ) denote the Schrödinger equation $H_{-} \Psi^{(-)}=\lambda \Psi^{(-)}$ (resp. $\left.H_{+} \Psi^{(+)}=\lambda \Psi^{(+)}\right)$.

Let DT be the transformation such that $\mathcal{L} \mapsto \widetilde{\mathcal{L}}, V_{-} \mapsto V_{+}, \Psi^{(-)} \mapsto \Psi^{(+)}$. Then the following statements holds:

i): $\operatorname{DT}\left(V_{-}\right)=V_{+}=\Psi_{\lambda_{1}}^{(-)} \partial_{x}^{2}\left(\frac{1}{\Psi_{\lambda_{1}}^{(-)}}\right)+\lambda_{1}=V_{-}-2 \partial_{x}^{2}\left(\ln \Psi_{\lambda_{1}}^{(-)}\right)$, $\operatorname{DT}\left(\Psi_{\lambda_{1}}^{(-)}\right)=\Psi_{\lambda_{1}}^{(+)}=\frac{1}{\Psi_{\lambda_{1}}^{(-)}}$, where $\Psi_{\lambda_{1}}^{(-)}$is a particular solution of $\mathcal{L}_{\lambda_{1}}$, $\lambda_{1} \in \Lambda$.

ii): $\operatorname{DT}\left(\Psi^{(-)}\right)=\Psi^{(+)}=\partial_{x} \Psi^{(-)}-\partial_{x}\left(\ln \Psi_{\lambda_{1}}^{(-)}\right) \Psi^{(-)}=\frac{W\left(\Psi_{\lambda_{1}}^{(-)}, \Psi^{(-)}\right)}{W\left(\Psi_{\lambda_{1}}^{(-)}\right)}, \lambda \neq \lambda_{1}$, where $\Psi^{(-)}=\Psi_{\lambda}^{(-)}$is the general solution of $\mathcal{L}_{\lambda}$ for $\lambda \in \Lambda \backslash\left\{\lambda_{1}\right\}$ and $\Psi^{(+)}=\Psi_{\lambda}^{(+)}$is the general solution of $\widetilde{\mathcal{L}}_{\lambda}$ also for $\lambda \in \Lambda \backslash\left\{\lambda_{1}\right\}$.

Proof. We treat each item separately. 
i): By hypothesis, we have $\operatorname{Card}(\Lambda) \geq 1, \lambda_{1} \in \Lambda$,

$H_{-} \Psi_{\lambda_{1}}^{(-)}=\lambda_{1} \Psi_{\lambda_{1}}^{(-)}$, and setting $\widetilde{H}_{ \pm}=H_{ \pm}-\lambda_{1}, \widetilde{V}_{ \pm}=V_{ \pm}-\lambda_{1}$ we obtain $\widetilde{H}_{-} \Psi_{\lambda_{1}}^{(-)}=0$. Thus, the superpotential $W=-\partial_{x} \ln \Psi_{\lambda_{1}}^{(-)}$, depending of $\lambda_{1}$, exists and satisfies

$$
\left(-\partial_{x}+W\right)\left(\partial_{x}+W\right) \Psi_{\lambda_{1}}^{(-)}=\widetilde{H}_{-} \Psi_{\lambda_{1}}^{(-)}=0 .
$$

Now, developing both sides of the expression, we have $\widetilde{V}_{-}=-\partial_{x} W+W^{2}$. So, interchanging operators we have

$$
\left(\partial_{x}+W\right)\left(-\partial_{x}+W\right) \Psi_{\lambda_{1}}^{(+)}=\widetilde{H}_{+} \Psi_{\lambda_{1}}^{(+)}=0,
$$

so that developing both sides of the expression we have $\widetilde{V}_{+}=\partial_{x} W+W^{2}$ which lead us to $W=\partial_{x} \ln \Psi_{\lambda_{1}}^{(+)}$. Thus, we obtain

$$
\operatorname{DT}\left(\Psi_{\lambda_{1}}^{(-)}\right)=\Psi_{\lambda_{1}}^{(+)}=\frac{1}{\Psi_{\lambda_{1}}^{(-)}}
$$

On another hand, as $\widetilde{V}_{+}-\widetilde{V}_{-}=V_{+}-V_{-}=2 \partial_{x} W, \widetilde{V}_{+}+\widetilde{V}_{-}=V_{+}+V_{-}-$ $2 \lambda_{1}=2 W^{2}$, we obtain $\mathrm{DT}\left(V_{-}\right)=V_{+}$which is given by

$$
V_{-}-2 \partial_{x}\left(\frac{\partial_{x} \Psi_{\lambda_{1}}^{(-)}}{\Psi_{\lambda_{1}}^{(-)}}\right)=2\left(\frac{\partial_{x} \Psi_{\lambda_{1}}^{(-)}}{\Psi_{\lambda_{1}}^{(-)}}\right)^{2}-V_{-}+2 \lambda_{1}=\Psi_{\lambda_{1}}^{(-)} \partial_{x}^{2}\left(\frac{1}{\Psi_{\lambda_{1}}^{(-)}}\right)+\lambda_{1} .
$$

ii): Suppose that $\operatorname{Card}(\Lambda)>1, \lambda \neq \lambda_{1}$ and $\Psi^{( \pm)}=\Psi_{\lambda}^{( \pm)}$satisfying

$$
H_{-} \Psi^{(-)}=\lambda \Psi^{(-)}, \quad \lambda \neq \lambda_{1},
$$

applying again the raising and lowering operators we have

$\left(-\partial_{x}+W\right)\left(\partial_{x}+W\right) \Psi^{(-)} \neq 0, \quad\left(\partial_{x}+W\right)\left(-\partial_{x}+W\right) \Psi^{(+)} \neq 0$,

so that

$$
\left(-\partial_{x}+W\right)\left(\partial_{x}+W\right) \Psi^{(-)}=\left(-\partial_{x}+W\right) \Psi^{(+)},
$$

we obtain

$$
\begin{aligned}
& \Psi^{(+)}=\left(\partial_{x}+W\right) \Psi^{(-)}=\partial_{x} \Psi^{(-)}+W \Psi^{(-)} \\
& =\partial_{x} \Psi^{(-)}-\frac{\partial_{x} \Psi_{\lambda_{1}}^{(-)}}{\Psi_{\lambda_{1}}^{(-)}} \Psi^{(-)}=\frac{W\left(\Psi_{\lambda_{1}}^{(-)}, \Psi^{(-)}\right)}{W\left(\Psi_{\lambda_{1}}^{(-)}\right)} .
\end{aligned}
$$

Now, we will show that effectively $\Psi^{(+)}$satisfy $H_{+} \Psi^{(+)}=\lambda \Psi^{(+)}$for $\lambda \neq \lambda_{1}$. Let suppose that

$$
\Psi^{(+)}=\partial_{x} \Psi^{(-)}-\Psi^{(-)} \frac{\partial_{x} \Psi_{\lambda_{1}}^{(-)}}{\Psi_{\lambda_{1}}^{(-)}}
$$

we can see that

$$
\partial_{x}^{2} \Psi^{(-)}-\Psi^{(-)} \frac{\partial_{x}^{2} \Psi_{\lambda_{1}}^{(-)}}{\Psi_{\lambda_{1}}^{(-)}}=\Psi^{(-)}\left(\lambda_{1}-\lambda\right)
$$


therefore, differentiating $\Psi^{(+)}$, we have

$$
\begin{aligned}
\partial_{x} \Psi^{(+)} & =\partial_{x}^{2} \Psi^{(-)}-\partial_{x} \Psi^{(-)} \frac{\partial_{x} \Psi_{\lambda_{1}}^{(-)}}{\Psi_{\lambda_{1}}^{(-)}}-\Psi^{(-)} \frac{\partial_{x}^{2} \Psi_{\lambda_{1}}^{(-)}}{\Psi_{\lambda_{1}}^{(-)}}+\Psi^{(-)}\left(\frac{\partial_{x} \Psi_{\lambda_{1}}^{(-)}}{\Psi_{\lambda_{1}}^{(-)}}\right)^{2} \\
& =\Psi^{(-)}\left(\lambda_{1}-\lambda\right)+\Psi^{(-)}\left(\frac{\partial_{x} \Psi_{\lambda_{1}}^{(-)}}{\Psi_{\lambda_{1}}^{(-)}}\right)^{2}-\partial_{x} \Psi^{(-)} \frac{\partial_{x} \Psi_{\lambda_{1}}^{(-)}}{\Psi_{\lambda_{1}}^{(-)}}
\end{aligned}
$$

By differentiating $\partial_{x} \Psi^{(+)}$, we have

$$
\begin{aligned}
& \partial_{x}^{2} \Psi^{(+)}=\partial_{x} \Psi^{(-)}\left(\lambda_{1}-\lambda\right)+2 \partial_{x} \Psi^{(-)}\left(\frac{\partial_{x} \Psi_{\lambda_{1}}^{(-)}}{\Psi_{\lambda_{1}}^{(-)}}\right)^{2}+2 \Psi^{(-)} \frac{\partial_{x} \Psi_{\lambda_{1}}^{(-)}}{\Psi_{\lambda_{1}}^{(-)}} \frac{\partial_{x}^{2} \Psi_{\lambda_{1}}^{(-)}}{\Psi_{\lambda_{1}}^{(-)}} \\
& -2 \Psi^{(-)}\left(\frac{\partial_{x} \Psi_{\lambda_{1}}^{(-)}}{\Psi_{\lambda_{1}}^{(-)}}\right)^{3}-\partial_{x}^{2} \Psi^{(-)} \frac{\partial_{x} \Psi_{\lambda_{1}}^{(-)}}{\Psi_{\lambda_{1}}^{(-)}}-\partial_{x} \Psi^{(-)} \frac{\partial_{x}^{2} \Psi_{\lambda_{1}}^{(-)}}{\Psi_{\lambda_{1}}^{(-)}} \\
& =\partial_{x} \Psi^{(-)}\left(\lambda_{1}-\lambda\right)+2 \partial_{x} \Psi^{(-)}\left(\frac{\partial_{x} \Psi_{\lambda_{1}}^{(-)}}{\Psi_{\lambda_{1}}^{(-)}}\right)^{2}-2 \Psi^{(-)}\left(\frac{\partial_{x} \Psi_{\lambda_{1}}^{(-)}}{\Psi_{\lambda_{1}}^{(-)}}\right)^{3}-\partial_{x} \Psi^{(-)} \frac{\partial_{x}^{2} \Psi_{\lambda_{1}}^{(-)}}{\Psi_{\lambda_{1}}^{(-)}} \\
& -\frac{\partial_{x} \Psi_{\lambda_{1}}^{(-)}}{\Psi_{\lambda_{1}}^{(-)}}\left(\partial_{x}^{2} \Psi^{(-)}-\Psi^{(-)} \frac{\partial_{x}^{2} \Psi_{\lambda_{1}}^{(-)}}{\Psi_{\lambda_{1}}^{(-)}}\right)+\Psi^{(-)} \frac{\partial_{x} \Psi_{\lambda_{1}}^{(-)}}{\Psi_{\lambda_{1}}^{(-)}} \frac{\partial_{x}^{2} \Psi_{\lambda_{1}}^{(-)}}{\Psi_{\lambda_{1}}^{(-)}} \\
& =\partial_{x} \Psi^{(-)}\left(\lambda_{1}-\lambda\right)+2 \partial_{x} \Psi^{(-)}\left(\frac{\partial_{x} \Psi_{\lambda_{1}}^{(-)}}{\Psi_{\lambda_{1}}^{(-)}}\right)^{2}-2 \Psi^{(-)}\left(\frac{\partial_{x} \Psi_{\lambda_{1}}^{(-)}}{\Psi_{\lambda_{1}}^{(-)}}\right)^{3}-\partial_{x} \Psi^{(-)} \frac{\partial_{x}^{2} \Psi_{\lambda_{1}}^{(-)}}{\Psi_{\lambda_{1}}^{(-)}} \\
& -\Psi^{(-)} \frac{\partial_{x} \Psi_{\lambda_{1}}^{(-)}}{\Psi_{\lambda_{1}}^{(-)}}\left(\lambda_{1}-\lambda\right)+\Psi^{(-)} \frac{\partial_{x} \Psi_{\lambda_{1}}^{(-)}}{\Psi_{\lambda_{1}}^{(-)}} \frac{\partial_{x}^{2} \Psi_{\lambda_{1}}^{(-)}}{\Psi_{\lambda_{1}}^{(-)}} \\
& =\left(\lambda_{1}-\lambda\right)\left(\partial_{x} \Psi^{(-)}-\Psi^{(-)} \frac{\partial_{x} \Psi_{\lambda_{1}}^{(-)}}{\Psi_{\lambda_{1}}^{(-)}}\right)-\frac{\partial_{x}^{2} \Psi_{\lambda_{1}}^{(-)}}{\Psi_{\lambda_{1}}^{(-)}}\left(\partial_{x} \Psi^{(-)}-\Psi^{(-)} \frac{\partial_{x} \Psi_{\lambda_{1}}^{(-)}}{\Psi_{\lambda_{1}}^{(-)}}\right) \\
& +2\left(\frac{\partial_{x} \Psi_{\lambda_{1}}^{(-)}}{\Psi_{\lambda_{1}}^{(-)}}\right)^{2}\left(\partial_{x} \Psi^{(-)}-\Psi^{(-)} \frac{\partial_{x} \Psi_{\lambda_{1}}^{(-)}}{\Psi_{\lambda_{1}}^{(-)}}\right) \\
& =\left(2\left(\frac{\partial_{x} \Psi_{\lambda_{1}}^{(-)}}{\Psi_{\lambda_{1}}^{(-)}}\right)^{2}-\frac{\partial_{x}^{2} \Psi_{\lambda_{1}}^{(-)}}{\Psi_{\lambda_{1}}^{(-)}}+\lambda_{1}-\lambda\right)\left(\partial_{x} \Psi^{(-)}-\Psi^{(-)} \frac{\partial_{x} \Psi_{\lambda_{1}}^{(-)}}{\Psi_{\lambda_{1}}^{(-)}}\right) \\
& =\left(2\left(\frac{\partial_{x} \Psi_{\lambda_{1}}^{(-)}}{\Psi_{\lambda_{1}}^{(-)}}\right)^{2}-\frac{\partial_{x}^{2} \Psi_{\lambda_{1}}^{(-)}}{\Psi_{\lambda_{1}}^{(-)}}+\lambda_{1}-\lambda\right) \Psi^{(+)} \\
& =\left(2\left(\frac{\partial_{x} \Psi_{\lambda_{1}}^{(-)}}{\Psi_{\lambda_{1}}^{(-)}}\right)^{2}-V_{-}+2 \lambda_{1}-\lambda\right) \Psi^{(+)},
\end{aligned}
$$

which implies that, for $\lambda \neq \lambda_{1}$, the function $\Psi^{(+)}$satisfies the Schrödinger equation $H_{+} \Psi^{(+)}=\lambda \Psi^{(+)}$,

$$
V_{+}=2\left(\frac{\partial_{x} \Psi_{\lambda_{1}}^{(-)}}{\Psi_{\lambda_{1}}^{(-)}}\right)^{2}-V_{-}+2 \lambda_{1}=V_{-}-2 \partial_{x}\left(\frac{\partial_{x} \Psi_{\lambda_{1}}^{(-)}}{\Psi_{\lambda_{1}}^{(-)}}\right)=\Psi_{\lambda_{1}}^{(-)} \partial_{x}^{2}\left(\frac{1}{\Psi_{\lambda_{1}}^{(-)}}\right)+\lambda_{1}
$$


Remark 4.2. According to the corollary of the general Darboux transformation, see section 1, we have $m=-\lambda, m_{1}=-\lambda_{1}, f(x)=V_{-}, y=\Psi^{(-)}$and $u=y=\Psi^{(+)}$. This gives us a faster proof of theorem 4.1

In agreement with the previous theorem, we obtain the following results.

Proposition 4.3. DT is isogaloisian and virtually strong isogaloisian. Furthermore, if $\partial_{x}\left(\ln \Psi_{\lambda_{1}}^{(-)}\right) \in K$, then DT is strong isogaloisian.

Proof. Let $K$ and $L_{\lambda}$ be respectively the coefficient field and the PicardVessiot extension of the equation $\mathcal{L}_{\lambda}$. Similarly, let $\widetilde{K}, \widetilde{L}_{\lambda}$ be the coefficient field and the Picard-Vessiot extension of the equation $\widetilde{\mathcal{L}}_{\lambda}$. As $\mathrm{DT}\left(V_{-}\right)=V_{+}=2 W^{2}-$ $V_{-}-2 \lambda_{1}$, where $W=-\partial_{x}\left(\ln \Psi_{\lambda_{1}}^{(-)}\right)$, we have $\widetilde{K}=K\left\langle\partial_{x}\left(\ln \Psi_{\lambda_{1}}^{(-)}\right)\right\rangle$. The Riccati equation $\partial_{x} W=V_{-}-W^{2}$ has one algebraic solution (Singer 1981, [57]), in this case $W=-\partial_{x}\left(\ln \Psi_{\lambda_{1}}^{(-)}\right)$. Let $\left\langle\Psi_{(1, \lambda)}^{(-)}, \Psi_{(2, \lambda)}^{(-)}\right\rangle$be a basis of solutions for equation $\mathcal{L}_{\lambda}$ and $\left\langle\Psi_{(1, \lambda)}^{(+)}, \Psi_{(2, \lambda)}^{(+)}\right\rangle$a basis of solutions for equation $\widetilde{\mathcal{L}}_{\lambda}$. Since the coefficient field for equation $\widetilde{\mathcal{L}}_{\lambda}$ is $\widetilde{K}=K\left\langle\partial_{x}\left(\ln \Psi_{\lambda_{1}}^{(-)}\right)\right\rangle$, we have $L=K\left\langle\Psi_{(1, \lambda)}^{(-)}, \Psi_{(2, \lambda)}^{(-)}\right\rangle$and

$$
\begin{gathered}
\widetilde{L}=\widetilde{K}\left\langle\Psi_{(1, \lambda)}^{(+)}, \Psi_{(2, \lambda)}^{(+)}\right\rangle=K\left\langle\Psi_{(1, \lambda)}^{(+)}, \Psi_{(2, \lambda)}^{(+)}, \partial_{x}\left(\ln \Psi_{\lambda_{1}}^{(-)}\right)\right\rangle \\
=K\left\langle\Psi_{(1, \lambda)}^{(-)}, \Psi_{(2, \lambda)}^{(-)}, \partial_{x}\left(\ln \Psi_{\lambda_{1}}^{(-)}\right)\right\rangle=\widetilde{K}\left\langle\Psi_{(1, \lambda)}^{(-)}, \Psi_{(2, \lambda)}^{(-)}\right\rangle,
\end{gathered}
$$

for $\lambda=\lambda_{1}$ and for $\lambda \neq \lambda_{1}$. Since $\partial_{x}\left(\ln \Psi_{\lambda_{1}}^{(-)}\right)$is algebraic over $K$, then

$$
\left(\operatorname{DGal}\left(L_{\lambda} / K\right)\right)^{0}=\left(\operatorname{DGal}\left(\widetilde{L}_{\lambda} / K\right)\right)^{0}, \quad \operatorname{DGal}\left(L_{\lambda} / K\right)=\operatorname{DGal}\left(\widetilde{L}_{\lambda} / \widetilde{K}\right),
$$

which means that DT is a virtually strong and isogaloisian transformation. In the case $\partial_{x}\left(\ln \Psi_{\lambda_{1}}^{(-)}\right) \in K$, then $\widetilde{K}=K$ and $\widetilde{L}=L$, which means that DT is a strong isogaloisian transformation.

Proposition 4.4. Consider Schrödinger operators $\mathfrak{L}_{\lambda}:=H_{-}-\lambda$ and $\widetilde{\mathfrak{L}}_{\lambda}:=H_{+}-\lambda$ such that $\mathrm{DT}\left(H_{-}-\lambda\right)=H_{+}-\lambda$. The eigenrings of $\mathfrak{L}_{\lambda}$ and $\widetilde{\mathfrak{L}}_{\lambda}$ are isomorphic.

Proof. Let $\mathcal{E}\left(\mathfrak{L}_{\lambda}\right)$ and $\mathcal{E}\left(\widetilde{\mathfrak{L}}_{\lambda}\right)$ denote the eigenrings of $\mathfrak{L}_{\lambda}$ and $\widetilde{\mathfrak{L}}_{\lambda}$ respectively. By proposition 4.3 the connected identity component of the Galois group is preserved by Darboux transformation. Let $T \in \mathcal{E}\left(\mathfrak{L}_{\lambda}\right)$. Let $\operatorname{Sol}\left(\mathfrak{L}_{\lambda}\right)$ and $\operatorname{Sol}\left(\widetilde{\mathfrak{L}}_{\lambda}\right)$ be the solution spaces for $\mathfrak{L}_{\lambda} \Psi^{(-)}=0$ and $\widetilde{\mathfrak{L}}_{\lambda} \Psi^{(-)}=0$ respectively. To transform $\mathcal{E}\left(\mathfrak{L}_{\lambda}\right)$ into $\mathcal{E}\left(\widetilde{\mathfrak{L}}_{\lambda}\right)$, we follow the commuting diagram:

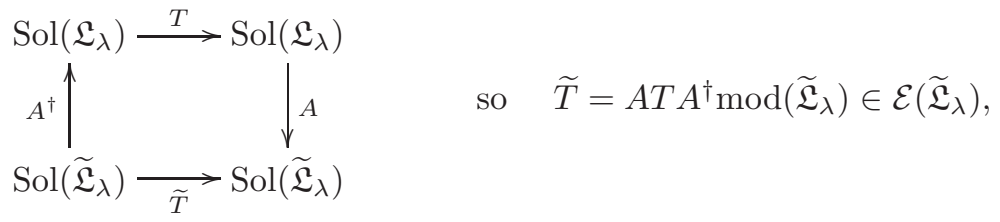

where $A^{\dagger}$ and $A$ are the raising and lowering operators. 
Example. Consider the Schrödinger equation $\mathcal{L}_{\lambda}$ with potential $V=V_{-}=0$, which means that $\Lambda=\mathbb{C}$. If we choose $\lambda_{1}=0$ and as particular solution $\Psi_{0}^{(-)}=x$, then for $\lambda \neq 0$ the general solution is given by

$$
\Psi_{\lambda}^{(-)}=c_{1} e^{\sqrt{-\lambda} x}+c_{2} e^{-\sqrt{-\lambda} x} .
$$

Applying the Darboux transformation DT, we have $\operatorname{DT}\left(\mathcal{L}_{\lambda}\right)=\widetilde{\mathcal{L}}_{\lambda}$, where

$$
\mathrm{DT}\left(V_{-}\right)=V_{+}=\frac{2}{x^{2}}
$$

and for $\lambda \neq 0$

$$
\operatorname{DT}\left(\Psi_{\lambda}^{(-)}\right)=\Psi_{\lambda}^{(+)}=\frac{c_{1}(\sqrt{-\lambda} x-1) e^{\sqrt{-\lambda} x}}{x}-\frac{c_{2}(\sqrt{-\lambda} x+1) e^{-\sqrt{-\lambda} x}}{x} .
$$

We can see that $\widetilde{K}=K=\mathbb{C}(x)$ for all $\lambda \in \Lambda$ and the Picard-Vessiot extensions are given by $L_{0}=\widetilde{L}_{0}=\mathbb{C}(x), L_{\lambda}=\widetilde{L}_{\lambda}=\mathbb{C}\left(x, e^{\sqrt{\lambda} x}\right)$ for $\lambda \in \mathbb{C}^{*}$. It follows that $\operatorname{DGal}\left(L_{0} / K\right)=\operatorname{DGal}\left(\widetilde{L}_{0} / K\right)=e$; for $\lambda \neq 0$, we have $\operatorname{DGal}\left(L_{\lambda} / K\right)=$ $\operatorname{DGal}\left(\widetilde{L}_{\lambda} / K\right)=\mathbb{G}_{m}$. The eigenrings of the operators $\mathfrak{L}_{0}$ and $\mathfrak{L}_{\lambda}$ are given by

$$
\begin{gathered}
\mathcal{E}\left(\mathfrak{L}_{0}\right)=\operatorname{Vect}\left(1, \partial_{x}, x \partial_{x}-1, x^{2} \partial_{x}-x\right), \\
\mathcal{E}\left(\widetilde{\mathfrak{L}}_{0}\right)=\operatorname{Vect}\left(1, x \partial_{x}-1, x^{4} \partial_{x}-2 x^{3}, \frac{\partial_{x}}{x^{2}}+\frac{1}{x^{3}}\right)
\end{gathered}
$$

and for $\lambda \neq 0$

$$
\mathcal{E}\left(\mathfrak{L}_{\lambda}\right)=\operatorname{Vect}\left(1, \partial_{x}\right), \quad \mathcal{E}\left(\widetilde{\mathfrak{L}}_{\lambda}\right)=\operatorname{Vect}\left(1,-\left(\lambda+\frac{1}{x^{2}}\right) \partial_{x}-\frac{1}{x^{3}}\right),
$$

where $\mathcal{L}_{\lambda}:=\mathfrak{L}_{\lambda} \Psi^{(-)}=0$ and $\widetilde{\mathcal{L}}_{\lambda}:=\widetilde{\mathfrak{L}}_{\lambda} \Psi^{(+)}=0$.

Applying iteratively the Darboux transformation, theorem 4.1, and by propositions 4.3, 4.4 we have the following results.

Proposition 4.5 (Galoisian version of $\mathrm{DT}_{n}$ ). We continue with the notations of theorem 4.1. We set $V_{0}=V_{-}, H^{(0)}=H_{-}, \Psi^{(0)}=\Psi^{(-)}$We still assume that the algebraic spectrum $\Lambda$ is non-empty. We consider the operators $\mathcal{L}_{\lambda}^{(n)}$ (given by $H^{(n)} \Psi^{(n)}=\lambda \Psi^{(n)}$ ) obtained by successive Darboux transformations. We let $K_{n}$ be the coefficient field of $\mathcal{L}_{\lambda}^{(n)}$ (i.e $V_{n} \in K_{n}$ ) and $K=K_{0}$. Let $L_{\lambda}^{(n)}$ denote the Picard-Vessiot extension of $\mathcal{L}_{\lambda}^{(n)}$. The Darboux transformation $\mathrm{DT}_{n}$ is such that $\mathcal{L}_{\lambda}^{(n)} \mapsto \mathcal{L}_{\lambda}^{(n+1)}, V_{n} \mapsto V_{n+1}, \Psi_{\lambda}^{(n)} \mapsto \Psi_{\lambda}^{(n+1)}$ and $L_{\lambda}^{(n+1)}$ the Picard-Vessiot extension of $\mathcal{L}_{\lambda}^{(n+1)}$.

Then the following statements hold:

i): $\operatorname{DT}_{n}\left(V_{-}\right)=\mathrm{DT}\left(V_{n}\right)=V_{n+1}=V_{n}-2 \partial_{x}^{2}\left(\ln \Psi_{\lambda}^{(n)}\right)=V_{-}-2 \sum_{k=0}^{n} \partial_{x}^{2}\left(\ln \Psi_{\lambda_{k}}^{(k)}\right)$, where $\Psi_{\lambda_{k}}^{(k)}$ is a particular solution for $\lambda=\lambda_{k}, k=0, \ldots, n$. In particular, if $\lambda_{n}=\lambda_{0}$ and $\Lambda=\mathbb{C}$, then there exists $\Psi_{\lambda_{n}}^{(n)}$ such that $V_{n} \neq V_{n-2}$, with $n \geq 2$.

ii): $\operatorname{DT}\left(\Psi_{\lambda}^{(n)}\right)=\operatorname{DT}_{n}\left(\Psi_{\lambda}^{(-)}\right)=\Psi_{\lambda}^{(n+1)}=\partial_{x} \Psi_{\lambda}^{(n)}-\Psi_{\lambda}^{(n)} \frac{\partial_{x} \Psi_{\lambda n}^{(n)}}{\Psi_{\lambda n}^{(n)}}=\frac{W\left(\Psi_{\lambda n}^{(n)}, \Psi_{\lambda}^{(n)}\right)}{W\left(\Psi_{\lambda n}^{(n)}\right)}$ where $\Psi_{\lambda}^{(n)}$ is a general solution for $\lambda \in \Lambda \backslash\left\{\lambda_{n}\right\}$ of $\mathcal{L}_{\lambda}^{(n)}$. 
iii): $K_{n+1}=K_{n}\left\langle\partial_{x}\left(\ln \Psi_{\lambda_{n}}^{(n)}\right)\right\rangle$.

iv): $\mathrm{DT}_{n}$ is isogaloisian and virtually strongly isogaloisian. Furthermore, if $\partial_{x}\left(\ln \Psi_{\lambda_{n}}^{(n)}\right) \in K_{n}$ then $\mathrm{DT}_{n}$ is strongly isogaloisian.

v): The eigenrings of $H^{(n)}-\lambda$ and $H^{(n+1)}-\lambda$ are isomorphic.

Proof. By induction on theorem 4.1 we obtain i) and ii). By induction on proposition 4.3 we obtain iii) and iv). By induction on proposition 4.4 we obtain v).

Example. Starting with $V=0$, the following potentials can be obtained using Darboux iteration $\mathrm{DT}_{n}$ (see [10, 12]).

$$
\begin{gathered}
\text { I) } V_{n}=\frac{n(n-1) b^{2}}{(b x+c)^{2}}, \quad \text { II) } V_{n}=\frac{m^{2} n(n-1)\left(b^{2}-a^{2}\right)}{(a \cosh (m x)+b \sinh (m x))^{2}}, \\
\text { III) } \left.V_{n}=\frac{-4 a b m^{2} n(n-1)}{\left(a e^{m x}+b e^{-m x}\right)^{2}}, \quad I V\right) V_{n}=\frac{m^{2} n(n-1)\left(b^{2}+a^{2}\right)}{(a \cos (m x)+b \sin (m x))^{2}} .
\end{gathered}
$$

In particular for the rational potential given in $I)$, we have $K=K_{n}=\mathbb{C}(x)$ and for $\lambda_{n}=\lambda=0$, we have

$$
\begin{gathered}
\Psi_{0}^{(n)}=\frac{c_{1}}{(b x+c)^{n}}+c_{2}(b x+c)^{n+1}, \text { so that } \operatorname{DGal}\left(L_{0} / K\right)=\operatorname{DGal}\left(L_{0}^{(n)} / K\right)=e, \\
\mathcal{E}\left(H^{(n)}\right)=\operatorname{Vect}\left(1, x \partial_{x}-1, x^{2 n+2} \partial_{x}-(n+1) x^{2 n+1}, \frac{\partial_{x}}{x^{2 n}}+\frac{n}{x^{2 n+1}}\right),
\end{gathered}
$$

whilst for $\lambda \neq 0$ and $\lambda_{n}=0$, the general solution $\Psi_{\lambda}^{(n)}$ is given by

$$
\Psi_{\lambda}^{(n)}(x)=c_{1} f_{n}(x, \lambda) h_{n}\left(\sin (\sqrt{\lambda} x)+c_{2} g_{n}(x, \lambda) j_{n}(\cos (\sqrt{\lambda} x),\right.
$$

where $f_{n}, g_{n}, h_{n}, j_{n} \in \mathbb{C}(x)$, so that

$$
\operatorname{DGal}\left(L_{\lambda} / K\right)=\operatorname{DGal}\left(L_{\lambda}^{(n)} / K\right)=\mathbb{G}_{m},
$$

and

$$
\operatorname{dim}_{\mathbb{C}} \mathcal{E}(H-\lambda)=\operatorname{dim}_{\mathbb{C}} \mathcal{E}\left(H^{(n)}-\lambda\right)=2 .
$$

\subsection{Crum Iteration.}

Proposition 4.6 (Galoisian version of $\mathrm{CI}_{n}$ ). Consider $\mathcal{L}_{\lambda}$ given by $H \Psi=\lambda \Psi$, $H=-\partial_{x}^{2}+V, V \in K$, such that $\operatorname{Card}(\Lambda)>n$ for a fixed $n \in \mathbb{Z}_{+}$. Let $\mathcal{L}_{\lambda}^{(n)}$ be given by $H^{(n)} \Psi^{(n)}=\lambda \Psi^{(n)}$, where $H^{(n)}=\partial_{x}^{2}+V_{n}, V_{n} \in K_{n}$. Let $\mathrm{CI}_{n}$ be the transformation such that $\mathcal{L}_{\lambda} \mapsto \mathcal{L}_{\lambda}^{(n)}, V \mapsto V_{n},\left(\Psi_{\lambda_{1}}, \ldots, \Psi_{\lambda_{n}}, \Psi_{\lambda}\right) \mapsto \Psi_{\lambda}^{(n)}$, where for $k=1, \ldots, n$ and the equation $\mathcal{L}_{\lambda}$, the function $\Psi_{\lambda}$ is the general solution for $\lambda \neq \lambda_{k}$ and $\Psi_{\lambda_{k}}$ is a particular solution for $\lambda=\lambda_{k}$. Then the following statements holds:

i): $\mathrm{CI}_{n}\left(\mathcal{L}_{\lambda}\right)=\mathcal{L}_{\lambda}^{(n)}$ where $\mathrm{CI}_{n}(V)=V_{n}=V-2 \partial_{x}^{2}\left(\ln W\left(\Psi_{\lambda_{1}}, \ldots, \Psi_{\lambda_{n}}\right)\right)$ and

$$
\mathrm{CI}_{n}\left(\Psi_{\lambda}\right)=\Psi_{\lambda}^{(n)}=\frac{W\left(\Psi_{\lambda_{1}}, \ldots, \Psi_{\lambda_{n}}, \Psi_{\lambda}\right)}{W\left(\Psi_{\lambda_{1}}, \ldots, \Psi_{\lambda_{n}}\right)}
$$

where $\Psi_{\lambda}^{(n)}$ is the general solution of $\mathcal{L}_{\lambda}^{(n)}$.

ii): $K_{n}=K\left\langle\partial_{x}\left(\ln W\left(\Psi_{\lambda_{1}}, \ldots, \Psi_{\lambda_{n}}\right)\right)\right\rangle$. 
iii): $\mathrm{CI}_{n}$ is isogaloisian and virtually strongly isogaloisian. Furthermore, if

$$
\partial_{x}\left(\ln W\left(\Psi_{\lambda_{1}}, \ldots, \Psi_{\lambda_{n}}\right)\right) \in K_{n},
$$

then $\mathrm{CI}_{n}$ is strongly isogaloisian.

iv): The eigenrings of $H-\lambda$ and $H^{(n)}-\lambda$ are isomorphic.

Proof. By induction on theorem 4.1 we obtain i). By induction on proposition 4.3 we obtain ii) and iii). By induction on proposition 4.4 we obtain iv).

Example. To illustrate the Crum iteration with rational potentials, we consider $V=\frac{2}{x^{2}}$. The general solution of $\mathcal{L}_{\lambda}:=H \Psi=\lambda \Psi$ is

$$
\frac{c_{1} e^{k x}(k x-1)}{x}+\frac{c_{2} e^{-k x}(k x+1)}{x}, \quad \lambda=-k^{2},
$$

the eigenfunctions for $\lambda_{1}=-1$, and $\lambda_{2}=-4$, are respectively given by

$$
\Psi_{-1}=\frac{e^{-x}(x+1)}{x}, \quad \Psi_{-4}=\frac{e^{-2 x}(2 x+1)}{2 x} .
$$

Thus, we obtain

$$
\mathrm{CI}_{2}(V)=V_{2}=\frac{8}{(2 x+3)^{2}}
$$

and the general solution of $\mathcal{L}_{\lambda}^{(2)}:=H^{(2)} \Psi^{(2)}=\lambda \Psi^{(2)}$ is

$$
\mathrm{CI}_{2}\left(\Psi_{\lambda}\right)=\Psi_{\lambda}^{(2)}=\frac{c_{1}(k(2 x+3)-2) e^{k x}}{2 x+3}+\frac{c_{2}(2+k(2 x+3)) e^{-k x}}{4 x+6}, \quad \lambda=-k^{2} .
$$

The differential Galois groups and eigenrings are given by:

$$
\operatorname{DGal}\left(L_{0} / K\right)=\operatorname{DGal}\left(L_{0}^{(2)} / K\right)=e, \quad \operatorname{dim}_{\mathbb{C}} \mathcal{E}(H)=\operatorname{dim}_{\mathbb{C}} \mathcal{E}\left(H^{(2)}\right)=4,
$$

and for $\lambda \neq 0$

$$
\operatorname{DGal}\left(L_{\lambda} / K\right)=\operatorname{DGal}\left(L_{\lambda}^{(2)} / K\right)=\mathbb{G}_{m}, \quad \operatorname{dim}_{\mathbb{C}} \mathcal{E}(H-\lambda)=\operatorname{dim}_{\mathbb{C}} \mathcal{E}\left(H^{(2)}-\lambda\right)=2 .
$$

Proposition 4.7. The supersymmetric partner potentials $V_{ \pm}$are rational functions if and only if the superpotential $W$ is a rational function.

Proof. The supersymmetric partner potentials $V_{ \pm}$are written as $V_{ \pm}=W^{2} \pm$ $\partial_{x} W$. We start considering the superpotential $W \in \mathbb{C}(x)$, so trivially we have $V_{ \pm} \in \mathbb{C}(x)$. Now assuming that $V_{ \pm} \in \mathbb{C}(x)$ we have $\partial_{x} W \in \mathbb{C}(x)$ and $W^{2} \in \mathbb{C}(x)$, which implies that $W \partial_{x} W \in \mathbb{C}(x)$ and therefore $W \in \mathbb{C}(x)$.

Corollary 4.8. The superpotential $W \in \mathbb{C}(x)$ if and only if $\mathrm{DT}$ is strong isogaloisian.

Proof. Assume that the superpotential $W \in \mathbb{C}(x)$. Thus, by proposition 4.3 DT is strong isogaloisian. Now, assume that DT is strong isogaloisian. Thus, $V_{ \pm} \in \mathbb{C}(x)$ and by proposition 4.7 we have $W \in \mathbb{C}(x)$. 
4.3. Shape Invariance. The following definition is a partial Galoisian adaptation of the original definition given in $[\mathbf{2 8}](K=\mathbb{C}(x))$. The complete Galoisian adaptation is given when $K$ is any differential field.

Definition 4.9 (Rational Shape Invariant Potentials). Assume $V_{ \pm}(x ; \mu) \in \mathbb{C}(x ; \mu)$, where $\mu$ is a family of parameters. The potential $V=V_{-} \in \mathbb{C}(x)$ is called a rational shape invariant potential with respect to $\mu$ and $E=E_{n}$ if there exists $f$ such that

$$
V_{+}\left(x ; a_{0}\right)=V_{-}\left(x ; a_{1}\right)+R\left(a_{1}\right), \quad a_{1}=f\left(a_{0}\right), \quad E_{n}=\sum_{k=2}^{n+1} R\left(a_{k}\right), \quad E_{0}=0 .
$$

Remark 4.10. We propose the following steps to check whether $V \in \mathbb{C}(x)$ is shape invariant.

Step 1.: Introduce parameters in $W(x)$ to obtain $W(x ; \mu)$, write $V_{ \pm}(x ; \mu)=$ $W^{2}(x ; \mu) \pm \partial_{x} W(x ; \mu)$, and replace $\mu$ by $a_{0}$ and $a_{1}$.

Step 2.: Obtain polynomials $\mathcal{P} \in \mathbb{C}\left[x ; a_{0}, a_{1}\right]$ and $\mathcal{Q} \in \mathbb{C}\left[x ; a_{0}, a_{1}\right]$ such that

$$
\partial_{x}\left(V_{+}\left(x ; a_{0}\right)-V_{-}\left(x ; a_{1}\right)\right)=\frac{\mathcal{P}\left(x ; a_{0}, a_{1}\right)}{\mathcal{Q}\left(x ; a_{0}, a_{1}\right)} .
$$

Step 3.: Set $\mathcal{P}\left(x ; a_{0}, a_{1}\right) \equiv 0$, as polynomial in $x$, to obtain $a_{1}$ in function of $a_{0}$, i.e., $a_{1}=f\left(a_{0}\right)$. Also obtain $R\left(a_{1}\right)=V_{+}\left(x ; a_{0}\right)-V_{-}\left(x ; a_{1}\right)$ and verify that exists $k \in \mathbb{Z}^{+}$such that $R\left(a_{1}\right)+\cdots+R\left(a_{k}\right) \neq 0$.

Example. Consider the superpotential of the three dimensional harmonic oscillator $W(r ; \ell)=r-\frac{\ell+1}{r}$. By step 1, the supersymmetric partner potentials are

$$
V_{-}(r ; \ell)=r^{2}+\frac{\ell(\ell+1)}{r^{2}}-2 \ell-3, \quad V_{+}(r ; \ell)=r^{2}+\frac{(\ell+1)(\ell+2)}{r^{2}}-2 \ell-1 .
$$

By step 2, we have $\partial_{r}\left(V_{+}\left(r ; a_{0}\right)-V_{-}\left(r ; a_{1}\right)\right)=-2 \frac{a_{0}^{2}+3 a_{0}-a_{1}^{2}-a_{1}+2}{r^{3}}$. By step 3, $\left(a_{0}+1\right)\left(a_{0}+2\right)=a_{1}\left(a_{1}+1\right)$, so that $a_{1}=f\left(a_{0}\right)=a_{0}+1, a_{n}=f\left(a_{n-1}\right)=a_{0}+n$, $R\left(a_{1}\right)=2$. Thus, we obtain the energy levels $E_{n}=2 n$ and the wave functions $\Psi_{n}^{(-)}(r ; \ell)=A^{\dagger}(r ; \ell) \cdots A^{\dagger}(r ; \ell+n-1) \Psi_{0}^{(-)}(r ; \ell+n)$, compare with [23.

By theorem 4.1 and propositions 4.3 .4 .4 and 4.7 we have the following result.

Theorem 4.11. Consider $\mathcal{L}_{n}:=H \Psi^{(-)}=E_{n} \Psi^{(-)}$with Picard-Vessiot extension $L_{n}$, where $n \in \mathbb{Z}_{+}$. If $V=V_{-} \in \mathbb{C}(x)$ is a shape invariant potential with respect to $E=E_{n}$, then

$$
\operatorname{DGal}\left(L_{n+1} / K\right)=\operatorname{DGal}\left(L_{n} / K\right), \quad \mathcal{E}\left(H-E_{n+1}\right) \simeq \mathcal{E}\left(H-E_{n}\right), \quad n>0 .
$$

Remark 4.12. As $W \in \mathbb{C}(x)$, a differential automorphism $\sigma$ commutes with the raising and lowering operators $A$ and $A^{\dagger}$. Furthermore the wave functions $\Psi_{n}^{(-)}$ can be written as $\Psi_{n}^{(-)}=P_{n} f_{n} \Psi_{0}^{(-)}$, where $P_{n}$ is a polynomial of degree $n$ in $x$ and $f_{n}$ is a sequence of functions with $f_{0}(x)=1$ such as was shown in the case of Harmonic oscillators and Coulomb potentials. 


\section{Schrödinger Equations with Rational Potentials}

Along this section we take $K=\mathbb{C}(x)$ to be our coefficient field. So all our differential operators will have coefficients in $K=\mathbb{C}(x)$.

5.1. Polynomial Potentials. We start by considering the Schrödinger equation (3.1) with polynomial potentials, i.e. $V \in \mathbb{C}[x]$, see [13, 64. For simplicity and without loss of generality, we consider monic polynomials (if the polynomial is $c_{n} x^{n}+\ldots+c_{1} x+c_{0}$, apply the transformation $\left.x \mapsto \sqrt[n+2]{c_{n}} x\right)$.

When $V$ is a polynomial of odd degree, is well known that the differential Galois group of such a Schrödinger equation (3.1) is $\mathrm{SL}(2, \mathbb{C})$, see [36].

We present here the complete result for the Schrödinger equation 3.1 with non-constant polynomial potential (Theorem [5.2), see also [4, §2]. The following lemma is useful for our purposes.

Lemma 5.1 (Completing Squares, 4). Every even degree monic polynomial of can be written in one only way completing squares, that is,

$$
Q_{2 n}(x)=x^{2 n}+\sum_{k=0}^{2 n-1} q_{k} x^{k}=\left(x^{n}+\sum_{k=0}^{n-1} a_{k} x^{k}\right)^{2}+\sum_{k=0}^{n-1} b_{k} x^{k},
$$

where

$$
\begin{gathered}
a_{n-1}=\frac{q_{2 n-1}}{2}, \quad a_{n-2}=\frac{q_{2 n-2}-a_{n-1}^{2}}{2}, \quad a_{n-3}=\frac{q_{2 n-3}-2 a_{n-1} a_{n-2}}{2}, \cdots, \\
a_{0}=\frac{q_{n}-2 a_{1} a_{n-1}-2 a_{2} a_{n-2}-\cdots}{2}, \quad b_{0}=q_{0}-a_{0}^{2}, \quad b_{1}=q_{1}-2 a_{0} a_{1}, \quad \cdots, \\
b_{n-1}=q_{n-1}-2 a_{0} a_{n-1}-2 a_{1} a_{n-2}-\cdots
\end{gathered}
$$

Proof. See lemma 2.4 in [4, p. 275].

We remark that $V(x)$ as in equation (5.1) can be written in terms of the superpotential $W(x)$, i.e., $V(x)=W^{2}(x)-\partial_{x} W(x)$, when

$$
n x^{n-1}+\sum_{k=1}^{n-1} k a_{k} x^{k-1}=-\sum_{k=0}^{n-1} b_{k} x^{k}
$$

and $W(x)$ is given by

$$
x^{n}+\sum_{k=0}^{n-1} a_{k} x^{k} .
$$

The following theorem also can be found in [4, §2], see also [3. Here we present a quantum mechanics adapted version.

Theorem 5.2 (Polynomial potentials and Galois groups, [4]). Let us consider the Schrödinger equation (3.1), with $V(x) \in \mathbb{C}[x]$ a polynomial of degree $k>0$. Then, its differential Galois group $\mathrm{DGal}\left(L_{\lambda} / K\right)$ falls in one of the following cases: 
(1) $\operatorname{DGal}\left(L_{\lambda} / K\right)=\mathrm{SL}(2, \mathbb{C})$,

(2) $\operatorname{DGal}\left(L_{\lambda} / K\right)=\mathbb{B}$,

and the eigenring of $H-\lambda$ is trivial, i.e., $\mathcal{E}(H-\lambda)=\operatorname{Vect}(1)$. Furthermore, $\operatorname{DGal}\left(L_{\lambda} / K\right)=\mathbb{B}$ if and only if the following conditions hold:

(1) $V(x)-\lambda$ is a polynomial of degree $k=2 n$ writing in the form of equation (5.1).

(2) $b_{n-1}-n$ or $-b_{n-1}-n$ is a positive even number $2 m, m \in \mathbb{Z}_{+}$.

(3) There exist a monic polynomial $P_{m}$ of degree $m$, satisfying

$$
\begin{aligned}
\partial_{x}^{2} P_{m}+2\left(x^{n}+\sum_{k=0}^{n-1} a_{k} x^{k}\right) \partial_{x} P_{m}+\left(n x^{n-1}+\sum_{k=0}^{n-2}(k+1) a_{k+1} x^{k}-\sum_{k=0}^{n-1} b_{k} x^{k}\right) P_{m}=0, \\
\text { or } \\
\partial_{x}^{2} P_{m}-2\left(x^{n}+\sum_{k=0}^{n-1} a_{k} x^{k}\right) \partial_{x} P_{m}-\left(n x^{n-1}+\sum_{k=0}^{n-2}(k+1) a_{k+1} x^{k}+\sum_{k=0}^{n-1} b_{k} x^{k}\right) P_{m}=0 .
\end{aligned}
$$

In such cases, the only possibilities for eigenfunctions with rational superpotentials are given by

$$
\Psi_{\lambda}=P_{m} e^{f(x)}, \quad \text { or } \quad \Psi_{\lambda}=P_{m} e^{-f(x)}, \quad \text { where } f(x)=\frac{x^{n+1}}{n+1}+\sum_{k=0}^{n-1} \frac{a_{k} x^{k+1}}{k+1} .
$$

Proof. See theorem 2.5 in [4 p. 276].

An easy consequence of the above theorem is the following.

Corollary 5.3. Assume that $V(x)$ is an algebraically solvable polynomial potential. Then $V(x)$ is of degree 2.

Proof. Writing $V(x)-\lambda$ in the form of equation (5.1) we see that $b_{n-1}-n=$ $2 m$ or $-b_{n-1}-n=2 m$, where $m \in \mathbb{Z}_{+}$. Thus, the integrability of the Schrödinger equation with $\operatorname{Card}(\Lambda)>1$ is obtained when $b_{n-1}$ is constant, so $n=1$.

Remark 5.4. Given a polynomial potential $V(x)$ such that $\operatorname{Spec}_{p}(H) \cap \Lambda \neq \emptyset$, we can obtain bound states and normalized wave functions if and only if the potential $V(x)$ is a polynomial of degree $4 n+2$. Furthermore, one integrability condition of $H \Psi=\lambda \Psi$ for $\lambda \in \Lambda$ is that $b_{2 n}$ must be an odd integer. In particular, if the potential

$$
V(x)=x^{4 n+2 n}+\mu x^{2 n}, \quad n>0
$$

is a quasi-exactly solvable, then $\mu$ is an odd integer. For this kind of potentials, we obtain bound states only when $\mu$ is a negative odd integer.

On another hand, the non-constant polynomial potentials $V(x)$ of degree $4 n$ are associated to non-hermitian Hamiltonians and $\mathcal{P} \mathcal{T}$ invariance which are not considered here, see [7. Furthermore, one integrability condition of $H \Psi=\lambda \Psi$ for 
$\lambda \in \Lambda$ is that $b_{2 n-1}$ must be an even integer. In particular, if the Schrödinger equation

$$
H \Psi=\lambda \Psi, \quad V(x)=x^{4 n}+\mu x^{2 n-1}, \quad \lambda \in \Lambda
$$

is integrable, then $\mu$ is an even integer.

We present the following examples to illustrate the previous theorem and remark.

Weber's Equation and Harmonic Oscillator. The Schrödinger equation with potential $V(x)=x^{2}+q_{1} x+q_{0}$ corresponds to the Rehm form of the Weber's equation. By lemma 5.1 we have

$$
V(x)-\lambda=\left(x+a_{0}\right)^{2}+b_{0}, \quad a_{0}=q_{1} / 2, \quad b_{0}=q_{0}-q_{1}^{2} / 4-\lambda .
$$

So that we obtain $\pm b_{0}-1=2 m$, where $m \in \mathbb{Z}_{+}$. If $b_{0}$ is an odd integer, then

$\operatorname{DGal}\left(L_{\lambda} / K\right)=\mathbb{B}, \mathcal{E}(H-\lambda)=\operatorname{Vect}(1), \lambda \in \Lambda=\left\{ \pm(2 m+1)+q_{0}-q_{1}^{2} / 4: m \in \mathbb{Z}_{+}\right\}$

and the set of eigenfunctions is either

$$
\Psi_{\lambda}=P_{m} e^{\frac{1}{2}\left(x^{2}+q_{1} x\right)}, \text { or, } \Psi_{\lambda}=P_{m} e^{-\frac{1}{2}\left(x^{2}+q_{1} x\right)} .
$$

In the second case we have bound state wave function and $\operatorname{Spec}_{p}(H) \cap \Lambda=\operatorname{Spec}_{p}(H)=$ $\left\{E_{m}=2 m+1+q_{0}-q_{1}^{2} / 4: m \in \mathbb{Z}_{+}\right\}$, which is infinite. The polynomials $P_{m}$ are related with the Hermite polynomials $H_{m}$, 15, 31, 46.

In particular we have the harmonic oscillator potential

$$
V(x)=\frac{1}{4} \omega^{2} x^{2}-\frac{\omega}{2},
$$

and the Schrödinger equation $H \Psi=E \Psi$. Through the change of independent variable $x \mapsto \sqrt{\frac{2}{\omega}} x$ we obtain $V(x)=x^{2}-1$ and $\lambda=\frac{2}{\omega} E$, that is, $q_{1}=0$ and $q_{0}=-1$. It follows that $\Lambda=\left\{ \pm(2 m+1)-1: m \in \mathbb{Z}_{+}\right\}$and the set of eigenfunctions is either

$$
\Psi_{\lambda}=P_{m} e^{\frac{1}{2} x^{2}}, \text { or }, \Psi_{\lambda}=P_{m} e^{-\frac{1}{2} x^{2}},
$$

where as below, $\operatorname{DGal}\left(L_{\lambda} / K\right)=\mathbb{B}$ and $\mathcal{E}(H-\lambda)=\{1\}$ for all $\lambda \in \Lambda$. In the second case we have bound state wave function, $\operatorname{Spec}_{p}(H) \cap \Lambda=\operatorname{Spec}_{p}(H)=\Lambda_{+}=\{2 m$ : $\left.m \in \mathbb{Z}_{+}\right\}$and $P_{m}=H_{m}$. The wave functions of $H \Psi=E \Psi$ for the harmonic oscillator potential are given by

$$
\Psi_{m}=H_{m}\left(\sqrt{\frac{2}{\omega}} x\right) \Psi_{0}, \quad \Psi_{0}=e^{-\frac{\omega}{4} x^{2}}, \quad E=E_{m}=m \omega .
$$

Quartic and Sextic Anharmonic Oscillator. The Schrödinger equation with potential $V(x)=x^{4}+q_{3} x^{3}+q_{2} x^{2}+q_{1} x+q_{0}$ can be obtained through transformations of confluent Heun's equation, though we will not adress this here. By lemma 5.1 we have

$$
V(x)-\lambda=\left(x^{2}+a_{1} x+a_{0}\right)^{2}+b_{1} x+b_{0},
$$

where $a_{1}=q_{3} / 2, a_{0}=q_{2} / 2-a_{1}^{2} / 2, b_{1}=q_{1}-2 a_{0} a_{1}$ and $b_{0}=q_{0}-a_{0}^{2}-\lambda$. So we obtain $\pm b_{1}-2=2 m$, where $m \in \mathbb{Z}_{+}$. If $\Lambda \neq \emptyset$, then $b_{1}$ is an even integer, $P_{m}$ satisfy 
the relation A.1. and $\operatorname{DGal}\left(L_{\lambda} / K\right)=\mathbb{B}$ for all $\lambda \in \Lambda$. The set of eigenfunctions is either

$$
\Psi_{\lambda}=P_{m} e^{\frac{x^{3}}{3}+\frac{a_{1} x^{2}}{2}+a_{0} x}, \text { or, } \Psi_{\lambda}=P_{m} e^{-\left(\frac{x^{3}}{3}+\frac{a_{1} x^{2}}{2}+a_{0} x\right)},
$$

where $\lambda$ and $m$ are related, which means that $\Lambda$ is finite, i.e., the potential is algebraically quasi-solvable. In particular for $q_{3}=2 \imath l, q_{2}=l^{2}-2 k, q_{1}=2 \imath(l k-J)$ and $q_{0}=0$, we have the quartic anharmonic oscillator potential, which can be found in 7.

Now, considering the potentials $V(x, \mu)=x^{4}+4 x^{3}+2 x^{2}-\mu x$, again by lemma 5.1 we have

$$
V(x, \mu)-\lambda=\left(x^{2}+2 x-1\right)^{2}+(4-\mu) x-1-\lambda,
$$

so that $\pm(4-\mu)-2=2 n$, where $n \in \mathbb{Z}_{+}$and in consequence $\mu \in 2 \mathbb{Z}$. Such $\mu$ can be either $\mu=2-2 n$ or $\mu=2 n+6$, where $n \in \mathbb{Z}_{+}$. By theorem [5.2, there exists a monic polynomial $P_{n}$ satisfying respectively

$$
\begin{gathered}
\partial_{x}^{2} P_{n}+\left(2 x^{2}+4 x-2\right) \partial_{x} P_{n}+((\mu-2) x+3+\lambda) P_{n}=0, \quad \mu=2-2 n, \quad \text { or } \\
\partial_{x}^{2} P_{n}-\left(2 x^{2}+4 x-2\right) \partial_{x} P_{n}+((\mu-6) x-1+\lambda) P_{n}=0, \quad \mu=2 n+6
\end{gathered}
$$

for $\Lambda \neq \emptyset$. This algebraic relation between the coefficients of $P_{n}, \mu$ and $\lambda$ give us the set $\Lambda$ in the following way:

(1) Write $P_{n}=x^{n}+c_{n-1} x^{n-1}+\ldots+c_{0}$, where $c_{i}$ are unknown.

(2) Pick $\mu$ and replace $P_{n}$ in the algebraic relation (A.1) to obtain a polynomial of degree $n$ with $n+1$ undetermined coefficients involving $c_{0}, \ldots c_{n-1}$ and $\lambda$. Each of such coefficients must be zero.

(3) The term $n+1$ is linear in $\lambda$ and $c_{n-1}$, thus we write $c_{n-1}$ in terms of $\lambda$. After of the elimination of the term $n+1$, we replace $c_{n-1}$ in the term $n$ to obtain a quadratic polynomial in $\lambda$ and so on until we reach the constant term which is a polynomial $Q_{n+1}(\lambda)$ of degree $n+1$ in $\lambda$. It follows that $\Lambda=\left\{\lambda: Q_{n+1}(\lambda)=0\right\}$ and $c_{0}, \ldots, c_{n-1}$ are determined for each value of $\lambda$.

For $\mu=2 n+6$, we have:

$$
\begin{aligned}
& n=0, \quad V(x, 6), \quad P_{0}=1, \quad \Lambda=\{1\} \\
& n=1, \quad V(x, 8), \quad P_{1}=x+1 \mp \sqrt{2}, \quad \Lambda=\{3 \pm 2 \sqrt{2}\}
\end{aligned}
$$

and the set of eigenfunctions is

$$
\Psi_{\lambda, \mu}=P_{n} e^{-\frac{1}{3} x^{3}-x^{2}+x} .
$$

In the same way, we can obtain $\Lambda, P_{n}$ and $\Psi_{\lambda, \mu}$ for $\mu=2-2 n$. However, we do not have bound states, $\operatorname{Spec}_{p}(H) \cap \Lambda=\emptyset, \operatorname{DGal}\left(L_{\lambda} / K\right)=\mathbb{B}$ and $\mathcal{E}(H-\lambda)=\operatorname{Vect}(1)$ for all $\lambda \in \Lambda$.

The well known sextic anharmonic oscillator $x^{6}+q_{5} x^{5}+\cdots+q_{1} x+q_{0}$ can be treated in a similar way, obtaining bound states wave functions and the BenderDunne orthogonal polynomials, which corresponds to $Q_{n+1}(\lambda)$, i.e. we also recover the results of $[\mathbf{8}, \mathbf{2 9}, \mathbf{5 3}$. The Schrödinger equation with this potential, under suitable transformations, also falls in the class of confluent Heun equations. 
5.2. Rational Potentials and Kovacic's Algorithm. Here we apply Kovacic's algorithm (see appendix A) to solve the Schrödinger equation with rational (non-polynomial) potentials.

Three dimensional harmonic oscillator potential:

$$
V(r)=\frac{1}{4} \omega^{2} r^{2}+\frac{\ell(\ell+1)}{r^{2}}-\left(\ell+\frac{3}{2}\right) \omega, \quad \ell \in \mathbb{Z} .
$$

In this case, the Schrödinger equation is written as

$$
\partial_{r}^{2} \Psi=\left(\left(\frac{1}{2} \omega r\right)^{2}+\frac{\ell(\ell+1)}{r^{2}}-\left(\ell+\frac{3}{2}\right) \omega-E\right) \Psi .
$$

By the change $r \mapsto\left(\sqrt{\frac{2}{\omega}}\right) r$ we obtain the Schrödinger equation

$$
\partial_{r}^{2} \Psi=\left(r^{2}+\frac{\ell(\ell+1)}{r^{2}}-(2 \ell+3)-\lambda\right) \Psi, \quad \lambda=\frac{2}{\omega} E
$$

and, in order to apply Kovacic's algorithm, we set

$$
R=r^{2}+\frac{\ell(\ell+1)}{r^{2}}-(2 \ell+3)-\lambda .
$$

We can see that this equation could fall in case 1 , in case 2 or in case 4 of Kovacic's algorithm. We start discarding the case 2 because by step 1 (of Kovacic's algorithm) we should have conditions $c_{2}$ and $\infty_{3}$; so we should have $E_{c}=\{2,4+$ $4 \ell,-4 \ell\}$ and $E_{\infty}=\{-2\}$, and by step 2 , we would have $n=-4 \notin \mathbb{Z}_{+}$, so that $D=\emptyset$. So this Schrödinger equation never falls in case 2 . Now, we only work with case 1 ; by step 1 , conditions $c_{2}$ and $\infty_{3}$ are satisfied, so that

$$
[\sqrt{R}]_{c}=0, \quad \alpha_{c}^{ \pm}=\frac{1 \pm(2 \ell+1)}{2}, \quad[\sqrt{R}]_{\infty}=r, \quad \alpha_{\infty}^{ \pm}=\frac{\mp(\lambda+2 \ell+3)-1}{2} .
$$

By step 2 we have the following possibilities for $n \in \mathbb{Z}_{+}$and for $\lambda \in \Lambda$ :

$$
\begin{array}{lll}
\left.\Lambda_{++}\right) & n=\alpha_{\infty}^{+}-\alpha_{0}^{+}=-\frac{1}{2}(4 \ell+6+\lambda), & \lambda=-2 n-4 \ell-6, \\
\left.\Lambda_{+-}\right) & n=\alpha_{\infty}^{+}-\alpha_{0}^{-}=-\frac{1}{2}(4+\lambda), & \lambda=-2 n-4, \\
\left.\Lambda_{-+}\right) & n=\alpha_{\infty}^{-}-\alpha_{0}^{+}=\frac{\lambda}{2}, & \lambda=2 n, \\
\left.\Lambda_{--}\right) & n=\alpha_{\infty}^{-}-\alpha_{0}^{-}=\frac{1}{2}(4 \ell+2+\lambda), & \lambda=2 n-4 \ell-2,
\end{array}
$$

where $\Lambda_{++} \cup \Lambda_{+-} \cup \Lambda_{-+} \cup \Lambda_{--}=\Lambda$, which means that $\lambda=2 m, m \in \mathbb{Z}$. Now, for $\lambda \in \Lambda$, the rational function $\omega$ in Kovacic's algorithm is given by:

$$
\begin{array}{lll}
\left.\Lambda_{++}\right) & \omega=r+\frac{\ell+1}{r}, & R_{n}=r^{2}+\frac{\ell(\ell+1)}{r^{2}}+(2 \ell+3)+2 n, \\
\left.\Lambda_{+-}\right) & \omega=r-\frac{\ell}{r}, & R_{n}=r^{2}+\frac{\ell(\ell+1)}{r^{2}}-(2 \ell-1)+2 n, \\
\left.\Lambda_{-+}\right) & \omega=-r+\frac{\ell+1}{r}, & R_{n}=r^{2}+\frac{\ell(\ell+1)}{r^{2}}-(2 \ell+3)-2 n, \\
\left.\Lambda_{--}\right) & \omega=-r-\frac{\ell}{r}, & R_{n}=r^{2}+\frac{\ell(\ell+1)}{r^{2}}+(2 \ell-1)-2 n,
\end{array}
$$


where $R_{n}$ is the coefficient of the differential equation $\widetilde{\mathcal{L}}_{n}:=\partial_{r}^{2} \Psi=R_{n} \Psi$, which is integrable for every $n$. For every $\lambda \in \Lambda$, we can see that $\operatorname{DGal}\left(\widetilde{L}_{n} / K\right)=$ $\operatorname{DGal}\left(L_{\lambda} / K\right)$, where $\mathcal{L}_{\lambda}:=H \Psi=\lambda \Psi$.

By step 3, there exists a polynomial of degree $n$ satisfying the relation (A.1):

$$
\begin{array}{lll}
\left.\Lambda_{++}\right) & \partial_{r}^{2} P_{n}+2\left(r+\frac{\ell+1}{r}\right) \partial_{r} P_{n}-2 n P_{n}=0, \quad \lambda \in \Lambda_{-}, \\
\left.\Lambda_{+-}\right) & \partial_{r}^{2} P_{n}+2\left(r-\frac{\ell}{r}\right) \partial_{r} P_{n}-2 n P_{n}=0, \quad \lambda \in \Lambda_{-}, \\
\left.\Lambda_{-+}\right) & \partial_{r}^{2} P_{n}+2\left(-r+\frac{\ell+1}{r}\right) \partial_{r} P_{n}+2 n P_{n}=0, \quad \lambda \in \Lambda_{+}, \\
\left.\Lambda_{--}\right) & \partial_{r}^{2} P_{n}+2\left(-r-\frac{\ell}{r}\right) \partial_{r} P_{n}+2 n P_{n}=0, \quad \lambda \in \Lambda .
\end{array}
$$

These polynomials exist for all $\lambda \in \Lambda$ when their degrees are $n \in 2 \mathbb{Z}$, while for $n \in 2 \mathbb{Z}+1$, they exist only for the cases $\left.\Lambda_{-+}\right)$and $\Lambda_{--}$) with special conditions. Hence we have obtained the algebraic spectrum $\Lambda=2 \mathbb{Z}$, where $\Lambda_{++}=4 \mathbb{Z}_{-}, \Lambda_{+-}=$ $2 \mathbb{Z}_{-}, \Lambda_{-+}=4 \mathbb{Z}_{+}, \Lambda_{--}=2 \mathbb{Z}$.

The possibilities for eigenfunctions, considering only $\lambda \in 4 \mathbb{Z}$, are given by

$$
\begin{array}{lll}
\left.\Lambda_{++}\right) & \Psi_{n}(r)=r^{\ell+1} P_{2 n}(r) e^{\frac{r^{2}}{2}}, & \lambda \in \Lambda_{-}, \\
\left.\Lambda_{+-}\right) & \Psi_{n}(r)=r^{-\ell} P_{2 n}(r) e^{\frac{r^{2}}{2}}, & \lambda \in \Lambda_{-}, \\
\left.\Lambda_{-+}\right) & \Psi_{n}(r)=r^{\ell+1} P_{2 n}(r) e^{\frac{-r^{2}}{2}}, & \lambda \in \Lambda_{+}, \\
\left.\Lambda_{--}\right) & \Psi_{n}(r)=r^{-\ell} P_{2 n}(r) e^{\frac{-r^{2}}{2}}, & \lambda \in \Lambda .
\end{array}
$$

To obtain the point spectrum, we look for $\Psi_{n}$ satisfying the bound state conditions which is in only true for $\lambda \in \Lambda_{-+}$. With the change $r \mapsto \sqrt{\frac{\omega}{2}} r$, the point spectrum and ground state of the Schrödinger equation with the 3D-harmonic oscillator potential are respectively $\operatorname{Spec}_{p}(H)=\left\{E_{n}: n \in \mathbb{Z}_{+}\right\}$, for $E_{n}=2 n \omega$, where $\omega$ is the angular velocity, and

$$
\Psi_{0}=\left(\sqrt{\frac{\omega}{2}} r\right)^{\ell+1} e^{-\frac{\omega}{4} r^{2}} .
$$

The bound state wave functions are obtained as $\Psi_{n}=P_{2 n} \Psi_{0}$. Now, we can see that $\operatorname{DGal}\left(L_{0} / K\right)=\mathbb{B}$ and $\mathcal{E}(H)=\operatorname{Vect}(1)$. Since $\Psi_{n}=P_{2 n} \Psi_{0}$, for all $\lambda \in \Lambda$ we have $\operatorname{DGal}\left(L_{\lambda} / K\right)=\mathbb{B}$ and $\mathcal{E}(H-\lambda)=\operatorname{Vect}(1)$. In particular, $\operatorname{DGal}\left(L_{\lambda} / K\right)=\mathbb{B}$ and $\mathcal{E}(H-\lambda)=\operatorname{Vect}(1)$ for all $\lambda \in \operatorname{Spec}_{p}(H)$, where $\lambda=\frac{2}{\omega} E$.

We remark that the Schrödinger equation with the 3D-harmonic oscillator potential, through the changes $r \mapsto \frac{1}{2} \omega r^{2}$ and $\Psi \mapsto \sqrt{r} \Psi$, fall in the class of Whittaker differential equations in which the parameters are given by

$$
\kappa=\frac{(2 \ell+3) \omega+2 E}{4 \omega}, \quad \mu=\frac{1}{2} \ell+\frac{1}{4}
$$

By Martinet-Ramis theorem, we have integrability when $\pm \kappa \pm \mu$ is a half integer. These conditions coincides with our four sets $\Lambda_{ \pm \pm}$. 


\section{Coulomb potential:}

$$
V(r)=-\frac{\mathrm{e}^{2}}{r}+\frac{\ell(\ell+1)}{r^{2}}+\frac{\mathrm{e}^{4}}{4(\ell+1)^{2}}, \quad \ell \in \mathbb{Z}
$$

The Schrödinger equation in this case can be written as

$$
\partial_{r}^{2} \Psi=\left(\frac{\ell(\ell+1)}{r^{2}}-\frac{\mathrm{e}^{2}}{r}+\frac{\mathrm{e}^{4}}{4(\ell+1)^{2}}-E\right) \Psi .
$$

By the change $r \mapsto \frac{2(\ell+1)}{\mathrm{e}^{2}} r$ we obtain the Schrödinger equation

$$
\partial_{r}^{2} \Psi=\left(\frac{\ell(\ell+1)}{r^{2}}-\frac{2(\ell+1)}{r}+1-\lambda\right) \Psi, \quad \lambda=\frac{4(\ell+1)^{2}}{\mathrm{e}^{4}} E
$$

and in order to apply Kovacic algorithm, we set

$$
R=\frac{\ell(\ell+1)}{r^{2}}-\frac{2(\ell+1)}{r}+1-\lambda .
$$

First we analyze the case for $\lambda=1$ : we can see that this equation only could fall in case 2 or in case 4 of Kovacic's algorithm. We start discarding the case 2 because by step 1 we should have conditions $c_{2}$ and $\infty_{3}$. So we should have $E_{c}=\{2,4+4 \ell,-4 \ell\}$ and $E_{\infty}=\{1\}$ and by step 2 , we would have $n \notin \mathbb{Z}$. Thus, $n \notin \mathbb{Z}_{+}$and $D=\emptyset$, so the differential Galois group of this Schrödinger equation for $\lambda=1$ is $\mathrm{SL}(2, \mathbb{C})$.

Now, we analyze the case for $\lambda \neq 1$ : we can see that this equation could fall in case 1 , in case 2 or in case 4 . We start discarding the case 2 because by step 1 we should have conditions $c_{2}$ and $\infty_{3}$, so that we should have $E_{c}=\{2,4+4 \ell,-4 \ell\}$ and $E_{\infty}=\{0\}$. By step 2, we should have $n=2 \ell \in \mathbb{Z}_{+}$, so that $D=\{2 \ell\}$ and the rational function $\theta$ is $\theta=\frac{-2 \ell}{x}$, but we discard this case because there could only exist one polynomial of degree $2 \ell$ for a fixed $\ell$, and for instance, there could only exist one eigenstate and one eigenfunction for the Schrödinger equation. Thus,

Now, we only work with case 1 , by step 1 , conditions $c_{2}$ and $\infty_{3}$ are satisfied.

$$
[\sqrt{R}]_{c}=0, \quad \alpha_{c}^{ \pm}=\frac{1 \pm(2 \ell+1)}{2}, \quad[\sqrt{R}]_{\infty}=\sqrt{1-\lambda}, \quad \alpha_{\infty}^{ \pm}=\mp \frac{\ell+1}{\sqrt{1-\lambda}} .
$$

By step 2 we have the following possibilities for $n \in \mathbb{Z}_{+}$and for $\lambda \in \Lambda$ :

$$
\begin{array}{lll}
\left.\Lambda_{++}\right) & n=\alpha_{\infty}^{+}-\alpha_{0}^{+}=-(\ell+1)\left(1+\frac{1}{\sqrt{1-\lambda}}\right), & \lambda=1-\left(\frac{\ell+1}{\ell+1+n}\right)^{2}, \\
\left.\Lambda_{+-}\right) & n=\alpha_{\infty}^{+}-\alpha_{0}^{-}=-\frac{\ell+1}{\sqrt{1-\lambda}}+\ell, & \lambda=1-\left(\frac{\ell+1}{\ell-n}\right)^{2}, \\
\left.\Lambda_{-+}\right) \quad n=\alpha_{\infty}^{-}-\alpha_{0}^{+}=(\ell+1)\left(\frac{1}{\sqrt{1-\lambda}}-1\right), & \lambda=1-\left(\frac{\ell+1}{\ell+1+n}\right)^{2}, \\
\left.\Lambda_{--}\right) \quad n=\alpha_{\infty}^{-}-\alpha_{0}^{-}=\frac{\ell+1}{\sqrt{1-\lambda}}+\ell, & \lambda=1-\left(\frac{\ell+1}{\ell-n}\right)^{2} .
\end{array}
$$


We can see that $\lambda \in \Lambda_{-}$when $\lambda \leq 0$, while $\lambda \in \Lambda_{+}$when $0 \leq \lambda<1$. Furthermore:

$$
\begin{aligned}
& \left.\Lambda_{++}\right) \quad \ell \leq-1, \quad \lambda \in \begin{cases}\Lambda_{-}, & \ell \leq \frac{-n-2}{2} \\
\Lambda_{+}, & \frac{-n-2}{2} \leq \ell \leq-1\end{cases} \\
& \left.\Lambda_{+-}\right) \quad \ell>0, \quad \lambda \in \begin{cases}\Lambda_{-}, & \ell \geq \frac{n-1}{2} \\
\Lambda_{+}, & 0 \leq \ell \leq \frac{n-1}{2}\end{cases} \\
& \left.\Lambda_{-+}\right) \quad \ell \in \mathbb{Z}, \quad \lambda \in \begin{cases}\Lambda_{-}, & \ell \leq \frac{-n-2}{2} \\
\Lambda_{+}, & \ell \geq-1\end{cases} \\
& \left.\Lambda_{--}\right) \quad \ell>0, \quad \lambda \in \begin{cases}\Lambda_{-}, & \ell \geq \frac{n-1}{2} \\
\Lambda_{+}, & 0 \leq \ell \leq \frac{n-1}{2}\end{cases}
\end{aligned}
$$

So the possible algebraic spectrum can be $\Lambda=\Lambda_{++} \cup \Lambda_{+-} \cup \Lambda_{-+} \cup \Lambda_{--}$, that is

$$
\Lambda=\left\{1-\left(\frac{\ell+1}{\ell+1+n}\right)^{2}: n \in \mathbb{Z}_{+}\right\} \cup\left\{1-\left(\frac{\ell+1}{\ell-n}\right)^{2}: n \in \mathbb{Z}_{+}\right\},
$$

Now, for $\lambda \in \Lambda$, the rational function $\omega$ is given by:

$$
\begin{array}{llll}
\left.\Lambda_{++}\right) & \omega=\frac{\ell+1}{\ell+1+n}+\frac{\ell+1}{r}, & \lambda \in \Lambda_{++}, & R_{n}=\frac{\ell(\ell+1)}{r^{2}}-\frac{2(\ell+1)}{r}+\left(\frac{\ell+1}{\ell+1+n}\right)^{2}, \\
\left.\Lambda_{+-}\right) & \omega=\frac{\ell+1}{\ell-n}-\frac{\ell}{r}, & \lambda \in \Lambda_{+-}, & R_{n}=\frac{\ell(\ell+1)}{r^{2}}-\frac{2(\ell+1)}{r}+\left(\frac{\ell+1}{\ell-n}\right)^{2}, \\
\left.\Lambda_{-+}\right) & \omega=-\frac{\ell+1}{\ell+1+n}+\frac{\ell+1}{r}, & \lambda \in \Lambda_{-+}, & R_{n}=\frac{\ell(\ell+1)}{r^{2}}-\frac{2(\ell+1)}{r}+\left(\frac{\ell+1}{\ell+1+n}\right)^{2}, \\
\left.\Lambda_{--}\right) & \omega=-\frac{\ell+1}{\ell-n}-\frac{\ell}{r}, & \lambda \in \Lambda_{--}, & R_{n}=\frac{\ell(\ell+1)}{r^{2}}-\frac{2(\ell+1)}{r}+\left(\frac{\ell+1}{\ell-n}\right)^{2},
\end{array}
$$

where $R_{n}$ is the coefficient of the differential equation $\partial_{r}^{2} \Psi=R_{n} \Psi$, which is integrable for every $n$.

By step 3, there exists a polynomial of degree $n$ satisfying the relation (A.1),

$$
\begin{array}{lll}
\left.\Lambda_{++}\right) & \partial_{r}^{2} P_{n}+2\left(\frac{\ell+1}{\ell+1+n}+\frac{\ell+1}{r}\right) \partial_{r} P_{n}+\frac{2(\ell+1)}{r}\left(1+\frac{\ell+1}{\ell+1+n}\right) P_{n} & =0, \\
\left.\Lambda_{+-}\right) & \partial_{r}^{2} P_{n}+2\left(\frac{\ell+1}{\ell-n}-\frac{\ell}{r}\right) \partial_{r} P_{n}+\frac{2(\ell+1)}{r}\left(1-\frac{\ell+1}{\ell-n}\right) P_{n} & =0, \\
\left.\Lambda_{-+}\right) & \partial_{r}^{2} P_{n}+2\left(-\frac{\ell+1}{\ell+1+n}+\frac{\ell+1}{r}\right) \partial_{r} P_{n}+\frac{2(\ell+1)}{r}\left(1-\frac{\ell+1}{\ell+1+n}\right) P_{n} & =0, \\
\left.\Lambda_{--}\right) & \partial_{r}^{2} P_{n}+2\left(-\frac{\ell+1}{\ell-n}-\frac{\ell}{r}\right) \partial_{r} P_{n}+\frac{2(\ell+1)}{r}\left(1+\frac{\ell+1}{\ell-n}\right) P_{n} & =0 .
\end{array}
$$

These polynomials exist for every $\lambda \in \Lambda$ when $n \in \mathbb{Z}$, but $P_{0}=1$ is satisfied only for $\lambda \in \Lambda_{-+}$. Hence we have confirmed that the algebraic spectrum $\Lambda$ is given by equation (5.2). 
The possibilities for the eigenfunctions are given by

$$
\begin{aligned}
& \left.\Lambda_{++}\right) \quad \Psi_{n}(r)=r^{\ell+1} P_{n}(r) f_{n}(r) e^{r}, \quad f_{n}(r)=e^{\frac{-n r}{\ell+1+n}}, \quad \lambda \in \begin{cases}\Lambda_{-}, & \ell \leq \frac{-n-2}{2} \\
\Lambda_{+}, & \frac{-n-2}{2} \leq \ell \leq-1\end{cases} \\
& \left.\Lambda_{+-}\right) \quad \Psi_{n}(r)=r^{-\ell} P_{n}(r) f_{n}(r) e^{r}, \quad f_{n}(r)=e^{\frac{n+1}{\ell-n} r}, \quad \lambda \in \begin{cases}\Lambda_{-}, & \ell \geq \frac{n-1}{2} \\
\Lambda_{+}, & 0 \leq \ell \leq \frac{n-1}{2}\end{cases} \\
& \left.\Lambda_{-+}\right) \quad \Psi_{n}(r)=r^{\ell+1} P_{n}(r) f_{n}(r) e^{-r}, \quad f_{n}(r)=e^{\frac{n r}{\ell+1+n}}, \quad \lambda \in \begin{cases}\Lambda_{-}, & \ell \leq \frac{-n-2}{2} \\
\Lambda_{+}, & \ell \geq-1\end{cases} \\
& \left.\Lambda_{--}\right) \quad \Psi_{n}(r)=r^{-\ell} P_{n}(r) f_{n}(r) e^{-r}, \quad f_{n}(r)=e^{\frac{n+1}{n-\ell} r}, \quad \lambda \in \begin{cases}\Lambda_{-}, & \ell \geq \frac{n-1}{2} \\
\Lambda_{+}, & 0 \leq \ell \leq \frac{n-1}{2}\end{cases}
\end{aligned}
$$

but $\Psi_{n}$ should satisfy the bound state conditions which is only true for $\lambda \in \Lambda_{-+} \cap$ $\Lambda_{+}$, so that we choose $\Lambda_{-+} \cap \Lambda_{+}=\operatorname{spec}_{p}(H)$, that is

$$
\operatorname{spec}_{p}(H)=\left\{1-\left(\frac{\ell+1}{\ell+n+1}\right)^{2}: \quad n \in \mathbb{Z}_{+}, \quad \ell \geq-1\right\} .
$$

By the change $r \mapsto \frac{\mathrm{e}^{2}}{2(\ell+1)} r$, the point spectrum and ground state of the Schrödinger equation with Coulomb potential are respectively

$$
\operatorname{Spec}_{p}(H)=\left\{E_{n}: n \in \mathbb{Z}_{+}\right\}, \quad E_{n}=\frac{\mathrm{e}^{4}}{4}\left(\frac{1}{(\ell+1)^{2}}-\frac{1}{(\ell+1+n)^{2}}\right)
$$

and

$$
\Psi_{0}=\left(\frac{\mathrm{e}^{2}}{2(\ell+1)} r\right)^{\ell+1} e^{-\frac{\mathrm{e}^{2}}{2(\ell+1)} r} .
$$

The eigenstates are given by $\Psi_{n}=P_{n} f_{n} \Psi_{0}$, where

$$
f_{n}(r)=e^{\frac{n \mathrm{e}^{2} r}{2(\ell+1+n)(\ell+1)}} .
$$

Now, we can see that $\operatorname{DGal}\left(L_{0} / K\right)=\mathbb{B}$ and $\mathcal{E}(H)=\operatorname{Vect}(1)$. Since $\Psi_{n}=P_{2 n} f_{n} \Psi_{0}$, for all $\lambda \in \Lambda$ we have $\operatorname{DGal}\left(L_{\lambda} / K\right)=\mathbb{B}$ and $\mathcal{E}(H-\lambda)=\operatorname{Vect}(1)$. In particular, $\operatorname{DGal}\left(L_{\lambda} / K\right)=\mathbb{B}$ and $\mathcal{E}(H-\lambda)=\operatorname{Vect}(1)$ for all $E \in \operatorname{Spec}_{p}(H)$, where $E=\frac{\mathrm{e}^{4}}{4(\ell+1)^{2}} \lambda$.

We remark that, as in the three dimensional harmonic oscillator, the Schrödinger equation with the Coulomb potential, through the change

$$
r \mapsto \frac{\sqrt{-4(\ell+1)^{2} E+\mathrm{e}^{4}}}{\ell+1} r,
$$

falls in the class of Whittaker differential equation with the parameters given by

$$
\kappa=\frac{\mathrm{e}^{2}(\ell+1)}{\sqrt{-4(\ell+1)^{2} E+\mathrm{e}^{4}}}, \quad \mu=\ell+\frac{1}{2} .
$$


Applying Martinet-Ramis theorem, we can impose $\pm \kappa \pm \mu$ half integer, to coincides with our four sets $\Lambda_{ \pm \pm}$.

Remark 5.5. By direct application of Kovacic's algorithm we have:

- The Schrödinger equation (3.1) with potential

$$
V(x)=a x^{2}+\frac{b}{x^{2}}
$$

is integrable for $\lambda \in \Lambda$ when

$-a=0, \quad b=\mu(\mu+1), \quad \mu \in \mathbb{C}, \quad \Lambda=\mathbb{C}$,

$-a=1, \quad b=0, \quad \lambda \in \Lambda=2 \mathbb{Z}+1$,

$-a=1, \quad b=\ell(\ell+1), \quad \ell \in \mathbb{Z}^{*}, \Lambda=2 \mathbb{Z}+1$.

- The only rational potentials (up to transformations) in which the elements of the algebraic spectrum are placed at the same distance, belongs to the family of potentials given by

$$
V(x)=\sum_{k=-\infty}^{2} a_{k} x^{k}, \quad a_{2} \neq 0 .
$$

In particular, the set $\Lambda$ for the harmonic oscillator $(a=1, b=0)$ and $3 D$ harmonic oscillator $(a=1, b=\ell(\ell+1))$ satisfies this.

Proposition 5.6. Let $\mathcal{L}_{\lambda}$ be the Schrödinger equation (3.1) with $K=\mathbb{C}(x)$ and Picard-Vessiot extension $L_{\lambda}$. If $\operatorname{DGal}\left(L_{0} / K\right)$ is finite primitive, then $\operatorname{DGal}\left(L_{\lambda} / K\right)$ is not finite primitive for all $\lambda \in \Lambda \backslash\{0\}$.

Proof. Pick $V \in \mathbb{C}(x)$ such that $\mathcal{L}_{0}$ falls in case 3 of Kovacic algorithm, then $\circ u_{\infty} \geq 2$. Assume $t, s \in \mathbb{C}[x]$ such that $V=\frac{s}{t}$, then $\operatorname{deg}(t) \geq \operatorname{deg}(s)+2$ and $V-\lambda=\frac{s-\lambda t}{t}$. Now, for $\lambda \neq 0$ we have $\operatorname{deg}(s-\lambda t)=\operatorname{deg}(t)$ and therefore $\circ(V-\lambda)_{\infty}=0$. So, for $\lambda \neq 0$, the equation $\mathcal{L}_{\lambda}$ does not falls in case 3 of Kovacic algorithm and therefore $\operatorname{DGal}\left(L_{\lambda} / K\right)$ is not finite primitive.

Corollary 5.7. Let $\mathcal{L}_{\lambda}$ be the Schrödinger equation (3.1) with $K=\mathbb{C}(x)$ and Picard-Vessiot extension $L_{\lambda}$. If $\operatorname{Card}(\Lambda)>1$, then there is either zero or one value of $\lambda$ such that $\operatorname{DGal}\left(L_{\lambda} / K\right)$ is a finite primitive group.

Proof. Assume that $\operatorname{Card}(\Lambda)>1$. Thus, by proposition 5.6 the Schrödinger equation does not falls in case 3 of Kovacic's algorithm.

We note that the study of the differential Galois groups of the Schrödinger equation with the Coulomb potential had also been analyzed by Jean-Pierre Ramis ([51]) using his summability theory in the eighties of the past century.

\section{Transcendental Potentials and the Algebrization Method}

In supersymmetric quantum mechanics, there exists potentials which are not rational functions and, for this reason, it is difficult to apply our Galoisian approach from section 5. In this section, we address this problem and present some results concerning differential equations with non-rational coefficients. For these differential equations it is useful, whenever possible, to replace it by a new differential equation over the Riemann sphere $\mathbb{P}^{1}$ (that is, with rational coefficients). We will propose techniques to find such a change of variable and demonstrate, through 
numerous examples, how our approach allows us to handle many examples from quantum mechanics. The equation over $\mathbb{P}^{1}$ is called an algebraic form or algebrization of the original equation.

This algebraic form is already studied in the 19th century litterature (Liouville, Darboux), but the problem of obtaining an algebraic form (if it exists) of a given differential equation is in general not an easy task. Here we develop a new methodology using the concept of Hamiltonian change of variables. Such a change of variables allows us to compute the algebraic form of a large number of differential equations of different types. In particular, for second order linear differential equations, we can apply Kovacic's algorithm over the algebraic form to solve the original equation.

The following definition can be found in $9,69, \mathbf{7 0}$.

Definition 6.1 (Pullbacks of differential equations). Let $\mathfrak{L}_{1} \in K_{1}\left[\partial_{z}\right]$ and $\mathfrak{L}_{2} \in$ $K_{2}\left[\partial_{x}\right]$ be differential operators. The expression $\mathfrak{L}_{2} \otimes\left(\partial_{x}+v\right)$ refers to the operator whose solutions are the solutions of $\mathfrak{L}_{2}$ multiplied by the solution $e^{-\int v d x}$ of $\partial_{x}+v$.

- $\mathfrak{L}_{2}$ is a proper pullback of $\mathfrak{L}_{1}$ by means of $f \in K_{2}$ if the change of variable $z=f(x)$ changes $\mathfrak{L}_{1}$ into $\mathfrak{L}_{2}$.

- $\mathfrak{L}_{2}$ is a pullback (also known as weak pullback) of $\mathfrak{L}_{1}$ by means of $f \in K_{2}$ if there exists $v \in K_{2}$ such that $\mathfrak{L}_{2} \otimes\left(\partial_{x}+v\right)$ is a proper pullback of $\mathfrak{L}_{1}$ by means of $f$.

In case of compact Riemann surfaces, the geometric mechanism behind the algebrization is a ramified covering of compact Riemann surfaces, see [44, 43.

6.1. Second Order Linear Differential Equations. Some results presented in this subsection also can be found in [4, $\S 2$ ].

Proposition 6.2 (Change of the independent variable, 4). Let us consider the following equation, with coefficients in $\mathbb{C}(z)$ :

$$
\mathcal{L}_{z}:=\partial_{z}^{2} y+a(z) \partial_{z} y+b(z) y=0,
$$

and $\mathbb{C}(z) \hookrightarrow L$ the corresponding Picard-Vessiot extension. Let $(K, \delta)$ be a differential field with $\mathbb{C}$ as field of constants. Let $\theta \in K$ be a non-constant element. Then, by the change of variable $z=\theta(x)$, equation (6.1) is transformed in

$$
\mathcal{L}_{x}:=\partial_{x}^{2} r+\left(a(\theta) \partial_{x} \theta-\frac{\partial_{x}^{2} \theta}{\partial_{x} \theta}\right) \partial_{x} r+b(\theta)\left(\partial_{x} \theta\right)^{2} r=0, \quad \partial_{x}=\delta, \quad r=y \circ \theta .
$$

Let $K_{0} \subset K$ be the smallest differential field containing $\theta$ and $\mathbb{C}$. Then equation (6.2) is a differential equation with coefficients in $K_{0}$. Let $K_{0} \hookrightarrow L_{0}$ be the corresponding Picard-Vessiot extension. Assume that

$$
\mathbb{C}(z) \rightarrow K_{0}, \quad z \mapsto \theta
$$

is an algebraic extension, then

$$
\left(\operatorname{DGal}\left(L_{0} / K_{0}\right)\right)^{0}=(\operatorname{DGal}(L / \mathbb{C}(z)))^{0} .
$$

Proposition 6.3. Assume that $\mathcal{L}_{x}$ and $\mathcal{L}_{z}$ are as in proposition 6.2. Let $\varphi$ be the transformation given by

$$
\varphi:\left\{\begin{array}{l}
z \mapsto \theta(x) \\
\partial_{z} \mapsto \frac{1}{\partial_{x} \theta} \delta .
\end{array}\right.
$$


Then $\operatorname{DGal}\left(L_{0} / K_{0}\right) \simeq \operatorname{DGal}\left(L / K_{0} \cap L\right) \subset \operatorname{DGal}(L / \mathbb{C}(z))$. Furthermore, if $K_{0} \cap L$ is algebraic over $\mathbb{C}(z)$, then $\left(\operatorname{DGal}\left(L_{0} / K_{0}\right)\right)^{0} \simeq(\operatorname{DGal}(L / \mathbb{C}(x)))^{0}$.

Proof. By Proposition 6.2 the transformation $\varphi$ leads us to

$$
\mathbb{C}(z) \simeq \varphi(\mathbb{C}(z)) \hookrightarrow K_{0} .
$$

We identify $\mathbb{C}(z)$ with $\varphi(\mathbb{C}(z))$, so that we can view $\mathbb{C}(z)$ as a subfield of $K_{0}$ and then by the standard Galois theory diagram (see [33, 71]),

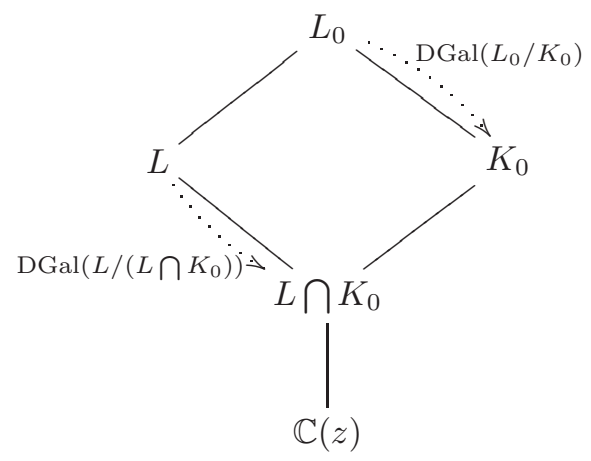

we have

$$
\operatorname{DGal}\left(L_{0} / K_{0}\right) \simeq \operatorname{DGal}\left(L / K_{0} \cap L\right) \subset \operatorname{DGal}(L / \mathbb{C}(z)) .
$$

If $K_{0} \cap L$ is algebraic over $\mathbb{C}(z)$, then

$$
\left(\operatorname{DGal}\left(L_{0} / K_{0}\right)\right)^{0} \simeq(\operatorname{DGal}(L / \mathbb{C}(z)))^{0} .
$$

For the remainder of this section we write $z=z(x)$ instead of $\theta$.

Remark 6.4 (Hard Algebrization). The proper pullback from equation (6.2) to equation (6.1) is an algebrization process. Therefore, we can try to algebrize any second order linear differential equations with non-rational coefficients (proper pullback) if we can put it in the form of equation (6.2). To do this, which will be called hard algebrization, we use the following steps.

Step 1: Find a term of the form $\left(\partial_{x} z\right)^{2}$ in the coefficient of $y$ to obtain a candidate for $\partial_{x} z$ and then $z$.

Step 2: Divide the coefficient of $y$ by $\left(\partial_{x} z\right)^{2}$ to obtain a candidate $b(z)$ and check whether $b \in \mathbb{C}(z)$.

Step 3: Add $\left(\partial_{x}^{2} z\right) / \partial_{x} z$ to the coefficient of $\partial_{x} y$ and divide the result by $\partial_{x} z$ to obtain a candidate $a(z)$ and check whether $a \in \mathbb{C}(z)$.

Let us illustrate this method of hard algebrization on the following example.

Example. In [58, p. 256], Singer presents the second order linear differential equation

$$
\partial_{x}^{2} r-\frac{1}{x(\ln x+1)} \partial_{x} r-(\ln x+1)^{2} r=0 .
$$


To algebrize this differential equation we choose $\left(\partial_{x} z\right)^{2}=(\ln x+1)^{2}$, so that $\partial_{x} z=$ $\ln x+1$ and for instance

$$
z=\int(\ln x+1) d x=x \ln x, \quad b(z)=-1 .
$$

Now we find $a(z)$ in the expression

$$
a(z)(\ln x+1)-\frac{1}{x(\ln x+1)}=-\frac{1}{x(\ln x+1)},
$$

obtaining $a(z)=0$. So that the new differential equation is given by $\partial_{z}^{2} y-y=0$, in which $y(z(x))=r(x)$ and one basis of solutions of this differential equation is given by $\left\langle e^{z}, e^{-z}\right\rangle$. Thus, the respective basis of solutions of the first differential equation is given by $\left\langle e^{x \ln x}, e^{-x \ln x}\right\rangle$.

In general, this method is strongly heuristic because the quest of $z=z(x)$ in $b(z)\left(\partial_{x} z\right)^{2}$ can be purely a lottery, or simply there may not exist $z$ such that $a(z), b(z) \in \mathbb{C}(z)$. For example, the equations presented by Singer in [58, p. 257, 261,270] and given by

$$
\begin{gathered}
\partial_{x}^{2} r+\frac{\mp 2 x \ln ^{2} x \mp 2 x \ln x-1}{x \ln x+x} \partial_{x} r+\frac{-2 x \ln ^{2} x-3 x \ln x-x \mp 1}{x \ln x+x} r=0, \\
\partial_{x}^{2} r+\frac{4 x \ln x+2 x}{4 x^{2} \ln x} \partial_{x} r-\frac{1}{4 x^{2} \ln x} r=0, \quad\left(x^{2} \ln ^{2} x\right) \partial_{x}^{2} r+\left(x \ln ^{2} x-3 x \ln x\right) \partial_{x} r+3 r=0,
\end{gathered}
$$
cannot be algebrized systematically with this method, although they correspond to pullbacks (not proper pullbacks) of differential equations with constant coefficients.

In [14, Bronstein and Fredet developed and implemented an algorithm to solve differential equations over $\mathbb{C}\left(x, e^{\int f}\right)$ without algebrizing them, see also [26. As an application of proposition 6.2 we have the following result 1 .

Proposition 6.5 (Linear differential equation over $\mathbb{C}\left(x, e^{\int f}\right)$, [4]). Let $f \in \mathbb{C}(x)$ be a rational function. Then, the differential equation

$$
\partial_{x}^{2} r-\left(f+\frac{\partial_{x} f}{f}-f e^{\int f} a\left(e^{\int f}\right)\right) \partial_{x} r+\left(f\left(e^{\int f}\right)\right)^{2} b\left(e^{\int f}\right) r=0,
$$

is algebrizable by the change $z=e^{\int f}$ and its algebraic form is given by

$$
\partial_{z}^{2} y+a(z) \partial_{z} y+b(z) y=0, \quad r(x)=y(z(x)) .
$$

Proof. Assume that $r(x)=y(z(x))$, and $z=z(x)=e^{\int f d x}$. We can see that $\partial_{x} z=f z, \quad \partial_{z} y=\frac{\partial_{x} r}{f z}, \quad \partial_{z}^{2} y=\frac{1}{f z} \partial_{x}\left(\frac{\partial_{x} r}{f z}\right)=\frac{1}{\left(f e^{f f}\right)^{2}}\left(\partial_{x}^{2} r-f+\left(\frac{\partial_{x} f}{f}\right)\right) \partial_{x} r$. Replacing in $\partial_{z}^{2} y+a(z) \partial_{z} y+b(z) y=0$ we obtain equation (6.3).

\footnotetext{
${ }^{1}$ This result is given in [4 $\left.\S 2\right]$, but we include here the proof for completeness.
} 
Example. The differential equation

$$
\partial_{x}^{2} r-\left(x+\frac{1}{x}-2 x e^{x^{2}}\right) \partial_{x} r+\lambda x^{2} e^{x^{2}} r=0,
$$

is algebrizable by the change $z=e^{\frac{x^{2}}{2}}$ and its algebraic form is given by

$$
\partial_{z}^{2} y+2 z \partial_{z} y+\lambda y=0 .
$$

Remark 6.6. According to proposition 6.5, we have the following cases.

(1) $f=n \frac{\partial_{x} h}{h}$, for a rational function $h, n \in \mathbb{Z}_{+}$, we have the trivial case, both equations are over the Riemann sphere and they have the same differential field, so that does not need to be algebrized.

(2) $f=\frac{1}{n} \frac{\partial_{x} h}{h}$, for a rational function $h, n \in \mathbb{Z}^{+},(6.3)$ is defined over an algebraic extension of $\mathbb{C}(x)$ and so that this equation is not necessarily over the Riemann sphere.

(3) $f \neq q \frac{\partial_{x} h}{h}$, for any rational function $h, q \in \mathbb{Q}$, (6.3) is defined over a transcendental extension of $\mathbb{C}(x)$ and so that this equation is not over the Riemann sphere.

To algebrize second order linear differential equations may be easier when the term in $\partial_{x} r$ is absent and the change of variable is Hamiltonian.

Definition 6.7 (Hamiltonian change of variable, 44). A change of variable $z=z(x)$ is called Hamiltonian if $\left(z(x), \partial_{x} z(x)\right)$ is a solution curve of the autonomous classical one degree of freedom Hamiltonian system

$$
\left\{\begin{array}{l}
\partial_{x} z=\partial_{w} H \\
\partial_{x} w=-\partial_{z} H
\end{array} \quad \text { with } \quad H=H(z, w)=\frac{w^{2}}{2}+V(z),\right.
$$

for some $V \in K$.

Remark 6.8. Assume that we algebrize equation (6.2) through a Hamiltonian change of variables $z=z(x)$, i.e. $V \in \mathbb{C}(z)$. Then $K_{0}=\mathbb{C}\left(z, \partial_{x} z, \ldots\right)$, but we have the algebraic relation

$$
\left(\partial_{x} z\right)^{2}=2 h-2 V(z), \quad h=H\left(z, \partial_{x} z\right) \in \mathbb{C},
$$

so that $K_{0}=\mathbb{C}\left(z, \partial_{x} z\right)$ is an algebraic extension of $\mathbb{C}(z)$. By proposition 6.2 the identity connected component of the differential Galois group is preserved. On the other hand, we can identify a Hamiltonian change of variable $z=z(x)$ when there exists $\alpha \in K$ such that $\left(\partial_{x} z\right)^{2}=\alpha(z)$. Thus, we introduce the Hamiltonian algebrization, which corresponds to the algebrization process done through a Hamiltonian change of variable.

The following result, which also can be found in [4, §2], is an example of Hamiltonian algebrization and correspond to the case of reduced second order linear differential equations.

Proposition 6.9 (Hamiltonian Algebrization, 4). The differential equation

$$
\partial_{x}^{2} r=q(x) r
$$

is algebrizable through a Hamiltonian change of variable $z=z(x)$ if and only if there exist $f, \alpha$ such that

$$
\frac{\partial_{z} \alpha}{\alpha}, \quad \frac{f}{\alpha} \in \mathbb{C}(z), \text { where } f(z(x))=q(x), \quad \alpha(z)=2(H-V(z))=\left(\partial_{x} z\right)^{2} .
$$


Furthermore, the algebraic form of the equation $\partial_{x}^{2} r=q(x) r$ is

$$
\partial_{z}^{2} y+\frac{1}{2} \frac{\partial_{z} \alpha}{\alpha} \partial_{z} y-\frac{f}{\alpha} y=0, \quad r(x)=y(z(x)) .
$$

Remark 6.10 (Using the Algebrization Method). The goal is to algebrize the differential equation $\partial_{x}^{2} r=q(x) r$. We propose the following steps.

Step 1: Look for a Hamiltonian change of variable $z=z(x)$ and two functions $f$ and $\alpha$ such that $q(x)=f(z(x))$ and $\left(\partial_{x} z(x)\right)^{2}=\alpha(z(x))$.

Step 2: Verify whether or not $f(z) / \alpha(z) \in \mathbb{C}(z)$ and $\partial_{z} \alpha(z) / \alpha(z) \in \mathbb{C}(z)$ to see if the equation $\partial_{x}^{2} r=q(x) r$ is algebrizable.

Step 3: If the equation $\partial_{x}^{2} r=q(x) r$ is algebrizable, its algebrization is

$$
\partial_{z}^{2} y+\frac{1}{2} \frac{\partial_{z} \alpha}{\alpha} \partial_{z} y-\frac{f}{\alpha} y=0, \quad y(z(x))=r(x) .
$$

When we have algebrized the differential equation $\partial_{x}^{2} r=q(x) r$, we study its integrability, its eigenring and its differential Galois group.

Examples. Consider the following examples.

- Given the differential equation $\partial_{x}^{2} r=f(\tan x) r$ with $f \in \mathbb{C}(\tan x)$, we can choose $z=z(x)=\tan x$ to obtain $\alpha(z)=\left(1+z^{2}\right)^{2}$, so that $z=z(x)$ is a Hamiltonian change of variable. We can see that $\frac{\partial_{z} \alpha}{\alpha}, \frac{f}{\alpha} \in \mathbb{C}(z)$ and the algebraic form of the differential equation $\partial_{x}^{2} r=f(\tan x) r$ with this Hamiltonian change of variable is

$$
\partial_{z}^{2} y+\frac{2 z}{1+z^{2}} \partial_{z} y-\frac{f(z)}{\left(1+z^{2}\right)^{2}} y=0, \quad y(\tan x)=r(x) .
$$

- Given the differential equation

$$
\partial_{x}^{2} r=\frac{\sqrt{1+x^{2}}+x^{2}}{1+x^{2}} r
$$

we can choose $z=z(x)=\sqrt{1+x^{2}}$ to obtain

$$
f(z)=\frac{z^{2}+z-1}{z^{2}}, \quad \alpha(z)=\frac{z^{2}-1}{z^{2}}
$$

so that $z=z(x)$ is a Hamiltonian change of variable. We can see that $\frac{\partial_{z} \alpha}{\alpha}, \frac{f}{\alpha} \in \mathbb{C}(z)$ and the algebraic form for this case is

$$
\partial_{z}^{2} y+\frac{1}{z\left(z^{2}-1\right)} \partial_{z} y-\frac{z^{2}+z-1}{z^{2}-1} y=0, \quad y\left(\sqrt{1+x^{2}}\right)=r(x) .
$$

We remark that in general the method of Hamiltonian algebrization is not an algorithm, because it may not be obvious to obtain a suitable Hamiltonian $H$ satisfying definition 6.7. We present now a particular case of Hamiltonian algebrization considered as an algorithm 2 .

Proposition 6.11 (Hamiltonian Algebrization Algorithm, 4). Consider $q(x)=$ $g\left(z_{1}, \cdots, z_{n}\right)$, where $z_{i}=e^{\lambda_{i} x}, \lambda_{i} \in \mathbb{C}^{*}$. The equation $\partial_{x}^{2} r=q(x) r$ is algebrizable if and only if.

$$
\frac{\lambda_{i}}{\lambda_{j}} \in \mathbb{Q}^{*}, \quad 1 \leq i \leq n, 1 \leq j \leq n, \quad g \in \mathbb{C}(z) .
$$

\footnotetext{
${ }^{2}$ Proposition 6.11 is a slight improvement of a similar result given in [4 $\left.\S 2\right]$. Furthermore, we include the proof here for completeness.
} 
Furthermore, $\lambda_{i}=c_{i} \lambda$, where $\lambda \in \mathbb{C}^{*}$ and $c_{i} \in \mathbb{Q}^{*}$ and for the Hamiltonian change of variable

$$
z=e^{\frac{\lambda x}{q}}, \text { where } c_{i}=\frac{p_{i}}{q_{i}}, p_{i}, q_{i} \in \mathbb{Z}^{*}, \operatorname{gcd}\left(p_{i}, q_{i}\right)=1 \text { and } q=\operatorname{lcm}\left(q_{1}, \cdots, q_{n}\right),
$$

the algebrization of the differential equation $\partial_{x}^{2} r=q(x) r$ is

$$
\partial_{z}^{2} y+\frac{1}{z} \partial_{z} y-q^{2} \frac{g\left(z^{m_{1}}, \ldots, z^{m_{n}}\right)}{\lambda^{2} z^{2}} y=0, \quad m_{i}=\frac{q p_{i}}{q_{i}}, \quad y(z(x))=r(x) .
$$

Proof. Assuming $\lambda_{i} / \lambda_{j}=c_{i j} \in \mathbb{Q}^{*}$ we can see that there exists $\lambda \in \mathbb{C}^{*}$ and $c_{i} \in \mathbb{Q}^{*}$ such that $\lambda_{i}=\lambda c_{i}$, so that

$$
e^{\lambda_{i} x}=e^{c_{i} \lambda x}=e^{\frac{p_{i}}{q_{i}} \lambda x}=\left(e^{\frac{\lambda}{q} x}\right)^{\frac{q p_{i}}{q_{i}}}, p_{i}, q_{i} \in \mathbb{Z}^{*}, \operatorname{gcd}\left(p_{i}, q_{i}\right)=1, \operatorname{lcm}\left(q_{1}, \ldots, q_{n}\right)=q .
$$

Now, setting $z=z(x)=e^{\frac{\lambda}{q} x}$ we can see that

$$
f(z)=g\left(z^{m_{1}}, \ldots, z^{m_{n}}\right), \quad m_{i}=\frac{q p_{i}}{q_{i}}, \quad \alpha=\frac{\lambda^{2} z^{2}}{q^{2}} .
$$

As $q \mid q_{i}$, we have $m_{i} \in \mathbb{Z}$, so that

$$
\frac{\partial_{z} \alpha}{\alpha}, \quad \frac{f}{\alpha} \in \mathbb{C}(z)
$$

and the algebraic form is given by

$$
\partial_{z}^{2} y+\frac{1}{z} \partial_{z} y-q^{2} \frac{g\left(z^{m_{1}}, \ldots, z^{m_{n}}\right)}{\lambda^{2} z^{2}} y=0, \quad y(z(x))=r(x) .
$$

Remark 6.12. Propositions 6.9 and 6.11 allow the algebrization of a large number of second order differential equations, see for example [49. In particular, under the assumptions of proposition 6.11, we can algebrize automatically differential equations with trigonometrical or hyperbolic coefficients.

Examples. Consider the following examples.

- Given the differential equation

$$
\partial_{x}^{2} r=\frac{e^{\frac{1}{2} x}+3 e^{-\frac{2}{3} x}-2 e^{\frac{5}{4} x}}{e^{x}+e^{-\frac{3}{2} x}} r, \lambda_{1}=\frac{1}{2}, \lambda_{2}=-\frac{2}{3}, \lambda_{3}=\frac{5}{4}, \lambda_{4}=1, \lambda_{5}=-\frac{3}{2},
$$

we see that $\lambda_{i} / \lambda_{j} \in \mathbb{Q}, \lambda=1, q=\operatorname{lcm}(1,2,3,4)=12$ and the Hamiltonian change of variable for this case is $z=z(x)=e^{\frac{1}{12} x}$. We can see that

$$
\alpha(z)=\frac{1}{144} z^{2}, \quad f(z)=\frac{z^{6}+3 z^{-8}-2 z^{15}}{z^{12}+z^{-18}}, \quad \frac{\partial_{z} \alpha}{\alpha}, \frac{f}{\alpha} \in \mathbb{C}(z)
$$

and the algebraic form is given by

$$
\partial_{z}^{2} y+\frac{1}{z} \partial_{z} y-144 \frac{z^{6}+3 z^{-8}-2 z^{15}}{z^{14}+z^{-16}} y=0, \quad y\left(e^{\frac{1}{12} x}\right)=r(x) .
$$

- Given the differential equation

$$
\partial_{x}^{2} r=\left(e^{2 \sqrt{2} x}+e^{-\sqrt{2} x}-e^{3 x}\right) r, \lambda_{1}=2 \sqrt{2}, \lambda_{2}=-\sqrt{2}, \lambda_{3}=3,
$$

we see that $\lambda_{1} / \lambda_{2} \in \mathbb{Q}$, but $\lambda_{1} / \lambda_{3} \notin \mathbb{Q}$, so that this differential equation cannot be algebrized. 
We remark that it is also possible to use the algebrization method to transform differential equations when either the starting equation has rational coefficients or the transformed equation does not have rational coefficients.

Examples. As an illustration, we treat the following examples.

- Consider the following differential equation:

$$
\partial_{x}^{2} r=\frac{x^{4}+3 x^{2}-5}{x^{2}+1} r=0 .
$$

We can choose $z=z(x)=x^{2}$ so that $\alpha=4 z$ and the new differential equation is

$$
\partial_{z}^{2} y+\frac{1}{2 z} \partial_{z} y-\frac{z^{2}+2 z-5}{4 z(z+1)} y=0, \quad y\left(x^{2}\right)=r(x) .
$$

- Consider the Mathieu differential equation $\partial_{x}^{2} r=(a+b \cos (x)) r$. We can choose $z(x)=\ln (\cos (x))$ so that $\alpha=e^{-2 z}-1$ and the new differential equation is

$$
\partial_{z}^{2} y-\frac{1}{1-e^{2 z}} \partial_{z} y-\frac{a e^{2 z}+b e^{3 z}}{1-e^{2 z}} y=0, \quad y(\ln (\cos (x)))=r(x) .
$$

Recently, the Hamiltonian algebrization (propositions 6.9 and 6.11) has been applied in [1, 2, 4, to obtain non-integrability results.

6.2. The Operator $\widehat{\partial}_{z}$ and the Hamiltonian Algebrization. The generalization of proposition 6.2 to higher order linear differential equations is difficult. But, it is possible to obtain generalizations of proposition 6.9 by means of Hamiltonian change of variable. We recall that $z=z(x)$ is a Hamiltonian change of variable if there exists $\alpha$ such that $\left(\partial_{x} z\right)^{2}=\alpha(z)$. More specifically, if $z=z(x)$ is a Hamiltonian change of variable, we can write $\partial_{x} z=\sqrt{\alpha}$, which leads us to the following notation: $\widehat{\partial}_{z}=\sqrt{\alpha} \partial_{z}$.

We can see that $\widehat{\partial}_{z}$ is a derivation because satisfy $\widehat{\partial}_{z}(f+g)=\widehat{\partial}_{z} f+\widehat{\partial}_{z} g$ and the Leibnitz rules

$$
\widehat{\partial}_{z}(f \cdot g)=\widehat{\partial}_{z} f \cdot g+f \cdot \widehat{\partial}_{z} g, \quad \widehat{\partial}_{z}\left(\frac{f}{g}\right)=\frac{\widehat{\partial}_{z} f \cdot g-f \cdot \widehat{\partial}_{z} g}{g^{2}} .
$$

We can notice that the chain rule is given by $\widehat{\partial}_{z}(f \circ g)=\partial_{g} f \circ g \widehat{\partial}_{z}(g) \neq \widehat{\partial}_{g} f \circ g \widehat{\partial}_{z}(g)$. The iteration of $\widehat{\partial}_{z}$ is given by

$$
\widehat{\partial}_{z}^{0}=1, \quad \widehat{\partial}_{z}=\sqrt{\alpha} \partial_{z}, \quad \widehat{\partial}_{z}^{n}=\sqrt{\alpha} \partial_{z} \widehat{\partial}_{z}^{n-1}=\underbrace{\sqrt{\alpha} \partial_{z}\left(\ldots\left(\sqrt{\alpha} \partial_{z}\right)\right)}_{n \text { times } \sqrt{\alpha} \partial_{z}} .
$$

We say that a Hamiltonian change of variable is rational when the potential $V \in$ $\mathbb{C}(x)$ and for instance $\alpha \in \mathbb{C}(x)$. For the remainder of this paper, we let $\widehat{\partial}_{z}=\sqrt{\alpha} \partial_{z}$ where $z=z(x)$ is a Hamiltonian change of variable and $\partial_{x} z=\sqrt{\alpha}$. In particular, $\widehat{\partial}_{z}=\partial_{z}=\partial_{x}$ if and only if $\sqrt{\alpha}=1$, i.e., $z=x$.

Theorem 6.13. Consider the systems of linear differential equations $[A]$ and $[\widehat{A}]$ given respectively by

$$
\partial_{x} \mathbf{Y}=-A \mathbf{Y}, \quad \widehat{\partial}_{z} \widehat{\mathbf{Y}}=-\widehat{A} \widehat{\mathbf{Y}}, \quad A=\left[a_{i j}\right], \quad \widehat{A}=\left[\widehat{a}_{i j}\right], \quad \mathbf{Y}=\left[y_{i 1}\right], \quad \widehat{\mathbf{Y}}=\left[\widehat{y}_{i 1}\right],
$$


where $a_{i j} \in K=\mathbb{C}\left(z(x), \partial_{x}(z(x))\right), \widehat{a}_{i j} \in \mathbb{C}(z) \subseteq \widehat{K}=\mathbb{C}(z, \sqrt{\alpha}), 1 \leq i \leq n$, $1 \leq j \leq n, a_{i j}(x)=\widehat{a}_{i j}(z(x))$ and $y_{i 1}(x)=y_{i 1}(z(x))$. Suppose that $L$ and $\widehat{L}$ are the Picard-Vessiot extensions of $[A]$ and $[\widehat{A}]$ respectively. If the transformation $\varphi$ is given by

$$
\varphi:\left\{\begin{array}{l}
x \mapsto z \\
a_{i j} \mapsto \widehat{a}_{i j} \\
y_{i 1}(x) \mapsto \widehat{y}_{i 1}(z(x)) \\
\partial_{x} \mapsto \widehat{\partial}_{z}
\end{array}\right.
$$

then the following statements hold.

- $K \simeq \widehat{K}, \quad\left(K, \partial_{x}\right) \simeq\left(\widehat{K}, \widehat{\partial}_{z}\right)$.

- $\operatorname{DGal}(L / K) \simeq \operatorname{DGal}(\widehat{L} / \widehat{K}) \subset \operatorname{DGal}(\widehat{L} / \mathbb{C}(z))$.

- $(\operatorname{DGal}(L / K))^{0} \simeq\left(\operatorname{DGal}(\widehat{L} / \mathbb{C}(z))^{0}\right.$.

- $\mathcal{E}(A) \simeq \mathcal{E}(\widehat{A})$.

Proof. We proceed as in the proof of proposition 6.3. As $z=z(x)$ is a rational Hamiltonian change of variable, the transformation $\varphi$ leads us to

$$
\mathbb{C}(z) \simeq \varphi(\mathbb{C}(z)) \hookrightarrow K, \quad K \simeq \widehat{K}, \quad \mathbb{C}(z) \hookrightarrow \widehat{K}, \quad\left(K, \partial_{x}\right) \simeq\left(\widehat{K}, \widehat{\partial}_{z}\right)
$$

that is, we identify $\mathbb{C}(z)$ with $\varphi(\mathbb{C}(z))$, and so that we can view $\mathbb{C}(z)$ as a subfield of $K$ and then, by the standard Galois theory diagram (see [33, 71]),

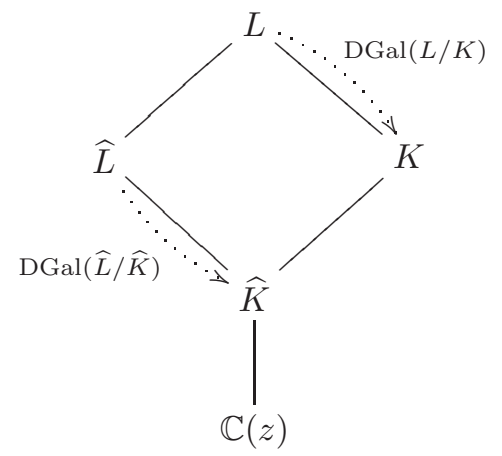

so that we have $\operatorname{DGal}(L / K) \simeq \operatorname{DGal}(\widehat{L} / \widehat{K}) \subset \operatorname{DGal}(\widehat{L} / \mathbb{C}(z)),(\operatorname{DGal}(L / K))^{0} \simeq$ $(\operatorname{DGal}(\widehat{L} / \mathbb{C}(z)))^{0}$, and $\mathcal{E}(A) \simeq \mathcal{E}(\widehat{A})$.

We remark that the transformation $\varphi$, given in theorem 6.13, is virtually strong isogaloisian when $\sqrt{\alpha} \notin \mathbb{C}(z)$ and for $\sqrt{\alpha} \in \mathbb{C}(z), \varphi$ is strong isogaloisian. Furthermore, by cyclic vector method (see $[\mathbf{6 5}]$ ), we can write the systems $[A]$ and $[\widehat{A}]$ in terms of the differential equations $\mathcal{L}$ and $\widehat{\mathcal{L}}$. Thus, $\widehat{\mathcal{L}}$ is the proper pullback of $\mathcal{L}$ and $\mathcal{E}(\mathfrak{L}) \simeq \mathcal{E}(\widehat{\mathfrak{L}})$.

Example. Consider the system

$$
[A]:=\left\{\begin{array}{l}
\partial_{x} \gamma_{1}=-\frac{2 \sqrt{2}}{e^{x}+e^{-x}} \gamma_{3}, \\
\partial_{x} \gamma_{2}=\frac{e^{x}-e^{-x}}{e^{x}+e^{-x}} \gamma_{3}, \\
\partial_{x} \gamma_{3}=\frac{2 \sqrt{2}}{e^{x}+e^{-x}} \gamma_{1}-\frac{e^{x}-e^{-x}}{e^{x}+e^{-x}} \gamma_{2}
\end{array}\right.
$$


Through the Hamiltonian change of variable $z=e^{x}$, and with $\sqrt{\alpha}=z$, it is transformed into the system

$$
\widehat{[A]}:=\left\{\begin{array}{l}
\partial_{z} \widehat{\gamma}_{1}=-\frac{2 \sqrt{2}}{z^{2}+1} \widehat{\gamma}_{3} \\
\partial_{z} \widehat{\gamma}_{2}=\frac{z^{2}-1}{z\left(z^{2}+1\right)} \widehat{\gamma}_{3} \\
\partial_{z} \widehat{\gamma}_{3}=\frac{2 \sqrt{2}}{z^{2}+1} \widehat{\gamma}_{1}-\frac{z^{2}-1}{x\left(x^{2}+1\right)} \widehat{\gamma}_{2}
\end{array} .\right.
$$

One solution of the system $\widehat{[A]}$ is given by

$$
\frac{1}{z^{2}+1}\left(\begin{array}{c}
\frac{\sqrt{2}}{2}\left(1-z^{2}\right) \\
z \\
-z
\end{array}\right),
$$

and the corresponding solution for the system $[A]$ is

$$
\frac{1}{e^{2 x}+1}\left(\begin{array}{c}
\frac{\sqrt{2}}{2}\left(1-e^{2 x}\right) \\
e^{x} \\
-e^{x}
\end{array}\right) .
$$

Remark 6.14. The algebrization given in proposition 6.9 is an example of how the introduction of the new derivative $\widehat{\partial}_{z}$ simplifies the proofs and computations. Such proposition is naturally extended to $\partial_{x}^{2} y+a \partial_{x} y+b y=0$, using $\varphi$ to obtain $\widehat{\partial}_{z}^{2} \widehat{y}+\widehat{a} \widehat{\partial}_{z} \widehat{y}+\widehat{b} \widehat{y}=0$, which is equivalent to

$$
\alpha \partial_{z}^{2} \widehat{y}+\left(\frac{\partial_{x} \alpha}{2}+\sqrt{\alpha} \widehat{a}\right) \partial_{z} \widehat{y}+\widehat{b} \widehat{y}=0,
$$

where $y(x)=\widehat{y}(z(x)), \widehat{a}(z(x))=a(x)$ and $\widehat{b}(z(x))=b(x)$.

In general, for $y(x)=\widehat{y}(z(x))$, the equation $F\left(\partial_{x}^{n} y, \ldots, y, x\right)=0$ with coefficients given by $a_{i_{k}}(x)$ is transformed into the equation $\widehat{F}\left(\widehat{\partial}_{z}^{n} \widehat{y}, \ldots, \widehat{y}, z\right)=0$ with coefficients given by $\widehat{a}_{i_{k}}(z)$, where $a_{i_{k}}(x)=\widehat{a}_{i_{k}}(z(x))$. In particular, for $\sqrt{\alpha}, \widehat{a}_{i_{k}} \in \mathbb{C}(z)$, the equation $\widehat{F}\left(\widehat{\partial}_{z}^{n} \widehat{y}, \ldots, \widehat{y}, z\right)=0$ is the Hamiltonian algebrization of $F\left(\partial_{x}^{n} y, \ldots, y, x\right)=0$. Now, if each derivation $\partial_{x}$ has even order, then $\alpha$ and $\widehat{a}_{i_{k}}$ can be rational functions when we algebrize the equation $F\left(\partial_{x}^{n} y, \ldots, y, x\right)=0$, where $a_{i_{k}} \in \mathbb{C}\left(z(x), \partial_{x} z(x)\right)$. For example, that happens for linear differential equations given by

$$
\partial_{x}^{2 n} y+a_{n-1}(x) \partial_{x}^{2 n-2} y+\ldots+a_{2}(x) \partial_{x}^{4} y+a_{1}(x) \partial_{x}^{2} y+a_{0}(x) y=0 .
$$

Finally, the algebrization algorithm given in proposition 6.11 can be naturally extended to a differential equation of the form

$$
F\left(\partial_{x}^{n} y, \partial_{x}^{n-1} y, \ldots, \partial_{x} y, y, e^{\mu t}\right)=0,
$$

which, through the change of variable $z=e^{\mu x}$, is transformed into

$$
\widehat{F}\left(\widehat{\partial}_{z}^{n} \widehat{y}, \widehat{\partial}_{z}^{n-1} \widehat{y}, \ldots, \widehat{\partial}_{z} \widehat{y}, y, z\right)=0 .
$$

In particular, we may consider the algebrization of Riccati equations, higher order linear differential equations and systems. 
Examples. The following corresponds to some examples of algebrizations for differential equations given in [58, p. 258, 266].

(1) The equation $\mathcal{L}:=\partial_{x}^{2} y+\left(-2 e^{x}-1\right) \partial_{x} y+e^{2 x} y=0$ with the Hamiltonian change of variable $z=e^{x}, \sqrt{\alpha}=z, \widehat{a}=-2 z-1$ and $\widehat{b}=z^{2}$ is transformed into the equation $\widehat{\mathcal{L}}:=\partial_{z}^{2} \widehat{y}-2 \partial_{z} \widehat{y}+\widehat{y}=0$ which can be easily solved. Bases of solutions for $\mathcal{L}$ and $\widehat{\mathcal{L}}$ are respectively given by $\left\langle e^{z}, z e^{z}\right\rangle$ and $\left\langle e^{e^{x}}, e^{x} e^{e^{x}}\right\rangle$. Furthermore $K=\mathbb{C}\left(e^{x}\right), \widehat{K}=\mathbb{C}(z), L$ and $\widehat{L}$ are the Picard-Vessiot extensions of $\mathcal{L}$ and $\widehat{\mathcal{L}}$ respectively. Thus, $\operatorname{DGal}(L / K)=\operatorname{DGal}(\widehat{L} / \widehat{K})$.

(2) The differential equation

$$
\mathcal{L}:=\partial_{x}^{2} y+\frac{-24 e^{x}-25}{4 e^{x}+5} \partial_{x} y+\frac{20 e^{x}}{4 e^{x}+5} y=0
$$

with the Hamiltonian change of variable $z=e^{x}, \sqrt{\alpha}=z$,

$$
\widehat{a}=\frac{-24 z-25}{4 z+5} \text { and } \widehat{b}=\frac{20 z}{4 z+5}
$$

is transformed into the equation

$$
\widehat{\mathcal{L}}:=\partial_{z}^{2} \widehat{y}+\frac{-20(z+1)}{x(4 z+5)} \partial_{z} \widehat{y}+\frac{20}{z(4 z+5)} \widehat{y}=0,
$$

which can be solved with Kovacic algorithm. A basis of solutions for $\widehat{\mathcal{L}}$ is $\left\langle z+1, z^{5}\right\rangle$, so that a basis for $\mathcal{L}$ is $\left\langle e^{x}+1, e^{5 x}\right\rangle$. Furthermore $K=$ $\mathbb{C}\left(e^{x}\right), \widehat{K}=\mathbb{C}(z), L$ and $\widehat{L}$ are the Picard-Vessiot extensions of $\mathcal{L}$ and $\widehat{\mathcal{L}}$ respectively. Thus, $\operatorname{DGal}(L / K)=\operatorname{DGal}(\widehat{L} / \widehat{K})=e$.

Remark 6.15 (Algebrization of the Riccati equation). The Riccati equation

$$
\partial_{x} v=a(x)+b(x) v+c(x) v^{2} .
$$

Through the Hamiltonian change of variable $z=z(x)$, it is transformed into the Riccati equation

$$
\partial_{z} \widehat{v}=\frac{1}{\sqrt{\alpha}}\left(\widehat{a}(z)+\widehat{b}(z) \widehat{v}+\widehat{c}(z) \widehat{v}^{2}\right),
$$

where $v(x)=\widehat{v}(z(x)), \widehat{a}(z(x))=a(x), \widehat{b}(z(x))=b(x), \widehat{c}(z(x))=c(x)$ and $\sqrt{\alpha(z(x))}=$ $\partial_{x} z(x)$. Furthermore, if $\sqrt{\alpha}, \widehat{a}, \widehat{b}, \widehat{c} \in \mathbb{C}(x)$, equation (6.7) is an algebrization of equation (6.6).

Example. Consider the Riccati differential equation

$$
\mathcal{L}:=\partial_{x} v=\left(\tanh x-\frac{1}{\tanh x}\right) v+\left(3 \tanh x-3 \tanh ^{3} x\right) v^{2},
$$

which through the Hamiltonian change of variable $z=\tanh x$, for instance $\sqrt{\alpha}=$ $1-z^{2}$, is transformed into the Riccati differential equation

$$
\widehat{\mathcal{L}}:=\partial_{z} v=-\frac{1}{z} v+3 z v^{2} .
$$

One solution for the equation $\widehat{\mathcal{L}}$ is

$$
-\frac{1}{z(3 z-c)}, \text { where } c \text { is a constant, }
$$


so that the corresponding solution for equation $\mathcal{L}$ is

$$
-\frac{1}{\tanh x(3 \tanh x-c)} \text {. }
$$

The following result is the algebrized version of the relationship between the eigenring s of systems and operators.

Proposition 6.16. Consider the differential fields $K, \widehat{K}$ and consider the systems $[A]$ and $[\widehat{A}]$ given by

$$
\partial_{x} \mathbf{X}=-A \mathbf{X}, \widehat{\partial}_{z} \widehat{\mathbf{X}}=-\widehat{A} \widehat{\mathbf{X}}, \widehat{\partial}_{z}=\sqrt{\alpha} \partial_{z}, A=\left[a_{i j}\right], \widehat{A}=\left[\widehat{a}_{i j}\right], a_{i j} \in K, \widehat{a}_{i j} \in \widehat{K},
$$

where $z=z(x), \mathbf{X}(x)=\widehat{\mathbf{X}}(z(x)), \widehat{a}_{i j}(z(x))=a_{i j}(x)$, then their eigenrings satisfy $\mathcal{E}(A) \simeq \mathcal{E}(\widehat{A})$. In particular, if we consider the linear differential equations

$$
\mathcal{L}:=\partial_{x}^{n} y+\sum_{k=0}^{n-1} a_{k} \partial_{x}^{k} y=0 \quad \text { and } \quad \widehat{\mathcal{L}}:=\widehat{\partial}_{z}^{n} \widehat{y}+\sum_{k=0}^{n-1} \widehat{a}_{k} \widehat{\partial}_{z}^{k} \widehat{y}=0
$$

where $z=z(x), y(x)=\widehat{z}((x)), \widehat{a}_{k}(z(x))=a_{k}(x), a_{k} \in K, \widehat{a}_{k} \in \widehat{K}$, then $\mathcal{E}(\mathfrak{L}) \simeq$ $\mathcal{E}(\widehat{\mathfrak{L}})$, where $\mathcal{L}:=\mathfrak{L} y=0$ and $\widehat{\mathcal{L}}:=\widehat{\mathfrak{L}} \widehat{y}=0$. Furthermore, assuming

$$
P=\left(\begin{array}{ccc}
p_{11} & \ldots & p_{1 n} \\
\vdots & & \\
p_{n 1} & \ldots & p_{n n}
\end{array}\right), \quad A=\left(\begin{array}{cccc}
0 & 1 & \ldots & 0 \\
\vdots & & & \\
-a_{0} & -a_{1} & \ldots & -a_{n-1}
\end{array}\right)
$$

then

if and only if

$$
\mathcal{E}(\mathfrak{L})=\left\{\sum_{k=1}^{n} p_{1 k} \partial_{x}^{k-1}: \partial_{x} P=P A-A P, p_{i k} \in K\right\},
$$

$$
\mathcal{E}(\widehat{\mathfrak{L}})=\left\{\sum_{k=1}^{n} \widehat{p}_{1 k} \widehat{\partial}_{z}^{k-1}: \widehat{\partial}_{z} \widehat{P}=\widehat{P} \widehat{A}-\widehat{A} \widehat{P}, \widehat{p}_{i k} \in \widehat{K}\right\} .
$$

Proof. By theorem 6.13 we have $K \simeq \widehat{K}, \mathcal{E}(A) \simeq \mathcal{E}(\widehat{A})$ and $\mathcal{E}(\mathfrak{L}) \simeq \mathcal{E}(\widehat{\mathfrak{L}})$. Using the derivation $\widehat{\partial}_{z}$ and by induction on lemma 6.3 we complete the proof.

Examples. We consider two different examples to illustrate the previous proposition.

- Consider the differential equation $\mathcal{L}_{1}:=\partial_{x}^{2} y-\left(1+\cos x-\cos ^{2} x\right) y=0$. By means of the Hamiltonian change of variable $z=z(x)=\cos x$, with $\sqrt{\alpha}=-\sqrt{1-z^{2}}, \mathcal{L}_{1}$ is transformed into the differential equation

$$
\widehat{\mathcal{L}}_{1}:=\partial_{z}^{2} \widehat{y}-\frac{z}{1-z^{2}} \partial_{z} \widehat{y}-\frac{1+z-z^{2}}{1-z^{2}} \widehat{y}=0 .
$$

Now, computing the eigenring of $\widehat{\mathfrak{L}}_{1}$ we have $\mathcal{E}\left(\widehat{\mathfrak{L}}_{1}\right)=\operatorname{Vect}(1)$, therefore the eigenring of $\mathfrak{L}_{1}$ is given $\mathcal{E}\left(\mathfrak{L}_{1}\right)=\operatorname{Vect}(1)$.

- Now we consider the differential equation $\mathcal{L}_{2}:=\partial_{x}^{2} y=\left(e^{2 x}+\frac{9}{4}\right) y$. By means of the Hamiltonian change of variable $z=e^{x}$, with $\sqrt{\alpha}=x, \mathcal{L}_{2}$ is transformed into the differential equation

$$
\widehat{\mathcal{L}}_{2}:=\partial_{z}^{2} \widehat{y}+\frac{1}{z} \partial_{z} \widehat{y}-\left(1+\frac{9}{4 x^{2}}\right) \widehat{y}=0 \text {. }
$$


Now, computing the eigenring of $\widehat{\mathfrak{L}}_{2}$ we have

$$
\mathcal{E}\left(\widehat{\mathfrak{L}}_{2}\right)=\operatorname{Vect}\left(1,-2\left(\frac{z^{2}-1}{z^{2}}\right) \partial_{z}-\frac{z^{2}-3}{z^{3}}\right)=\operatorname{Vect}\left(1,-2\left(\frac{z^{2}-1}{z^{3}}\right) \widehat{\partial}_{z}-\frac{z^{2}-3}{z^{3}}\right),
$$

therefore the eigenring of $\mathfrak{L}_{2}$ is given by

$$
\mathcal{E}\left(\mathfrak{L}_{2}\right)=\operatorname{Vect}\left(1,-2\left(\frac{e^{2 x}-1}{e^{3 x}}\right) \partial_{x}-\frac{e^{2 x}-3}{e^{3 x}}\right) .
$$

The same result is obtained via matrix formalism, where

$$
A=\left(\begin{array}{cc}
0 & 1 \\
e^{2 x}+\frac{9}{4} & 0
\end{array}\right), \widehat{A}=\left(\begin{array}{cc}
0 & 1 \\
z^{2}+\frac{9}{4} & 0
\end{array}\right), \partial_{x} P=P A-A P, \widehat{\partial}_{z} \widehat{P}=\widehat{P} \widehat{A}-\widehat{A} \widehat{P}
$$

with $P \in \operatorname{Mat}\left(2, \mathbb{C}\left(e^{x}\right)\right)$ and $\widehat{P} \in \operatorname{Mat}(2, \mathbb{C}(z))$.

6.3. Applications in Supersymmetric Quantum Mechanics. In this subsection we apply the derivation $\widehat{\partial}_{z}$ to the Schrödinger equation $H \Psi=\lambda \Psi$, where $H=-\partial_{x}^{2}+V(x), V \in K$. Assume that $z=z(x)$ is a rational Hamiltonian change of variable for $H \Psi=\lambda \Psi$, then $K=\mathbb{C}\left(z(x), \partial_{x} z(x)\right)$. Thus, the algebrized Schrödinger equation is written as

$$
\widehat{H} \widehat{\Psi}=\lambda \widehat{\Psi}, \quad \widehat{H}=-\widehat{\partial}_{z}^{2}+\widehat{V}(z), \quad \widehat{\partial}_{z}^{2}=\alpha \partial_{z}^{2}+\frac{1}{2} \partial_{z} \alpha \partial_{z}, \quad \widehat{K}=\mathbb{C}(z, \sqrt{\alpha}) .
$$

The reduced algebrized Schrödinger equation is given by

$$
\begin{array}{r}
\widehat{\mathbf{V}}(z)=\mathcal{V}+\frac{\widehat{V}(z)}{\alpha} \\
\mathcal{H}=\partial_{z} \mathcal{W}+\mathcal{W}^{2} \\
\mathcal{W}=\frac{1}{4} \frac{\partial_{z} \alpha(z)}{\alpha(z)}
\end{array}
$$

The eigenfunctions $\Psi, \widehat{\Psi}$ and $\Phi$ corresponding to the operators $H, \widehat{H}$ and $\widehat{\mathbf{H}}$ are related respectively by

$$
\Phi(z(x))=\sqrt[4]{\alpha} \widehat{\Psi}(z(x))=\sqrt[4]{\alpha} \Psi(x) .
$$

In order to apply the Kovacic's algorithm, we only consider the algebrized operator $\widehat{\mathbf{H}}$, whilst the eigenrings will be computed on $\widehat{H}$. Also it is possible to apply the version of Kovacic's algorithm given in reference $\mathbf{6 2}$ to the algebraized operator $\widehat{H}$. The following results are obtained by applying Kovacic's algorithm to the reduced algebrized Schrödinger equation (equation (6.9) $) \hat{\mathbf{H}} \Phi=\lambda \Phi$.

Proposition 6.17. Let $\widehat{\mathbf{L}}_{\lambda}$ be the Picard-Vessiot extension of the reduced algebrized Schrödinger equation $\widehat{\mathbf{H}} \Phi=\lambda \Phi$ with $\alpha, \widehat{V} \in \mathbb{C}[z]$. If $\operatorname{deg} \alpha<2+\operatorname{deg} \widehat{V}$ then, for every $\lambda \in \Lambda, \operatorname{DGal}(\widehat{\mathbf{L}} / \widehat{K})$ is not finite primitive.

Proof. Suppose that $\operatorname{deg} \alpha=n$ and $\operatorname{deg} \widehat{V}=m$. The reduced algebrized Schrödinger equation $\widehat{\mathbf{H}} \Phi=\lambda \Phi$ can be written in the form

$$
\partial_{z}^{2} \Phi=r \Phi, \quad r=\frac{4 \alpha \partial_{z}^{2} \alpha-3\left(\partial_{z} \alpha\right)^{2}+16 \alpha(\widehat{V}-\lambda)}{16 \alpha^{2}} .
$$

As $m>n-2$ we have $\circ\left(r_{\infty}\right)=n-m<2$, which does not satisfy the condition $(\infty)$ of case 3 of Kovacic's algorithm, therefore $\operatorname{DGal}(\widehat{\mathbf{L}} / \widehat{K})$ is not finite primitive for any $\lambda \in \Lambda$. 
Proposition 6.18. Let $\widehat{\mathbf{L}}_{\lambda}$ be the Picard-Vessiot extension of the reduced algebrized Schrödinger equation $\widehat{\mathbf{H}} \Phi=\lambda \Phi$ with $\alpha \in \mathbb{C}[z], \widehat{V} \in \mathbb{C}(z)$. If $\circ(\widehat{V})_{\infty}<2-\operatorname{deg} \alpha$ then, for every $\lambda \in \Lambda, \operatorname{DGal}\left(\widehat{\mathbf{L}}_{\lambda} / \widehat{K}\right)$ is not finite primitive.

Proof. Suppose that $\widehat{V}=s / t$, where $s$ and $t$ are coprime polynomials in $\mathbb{C}(z)$. Assume that $\operatorname{deg} \alpha=n, \operatorname{deg} s=m$ and $\operatorname{deg} t=p$. The reduced algebrized Schrödinger equation $\widehat{\mathbf{H}} \Phi=\lambda \Phi$ can be written in the form

$$
\partial_{z}^{2} \Phi=r \Phi, \quad r=\frac{4 t \alpha \partial_{z}^{2} \alpha-3 t\left(\partial_{z} \alpha\right)^{2}+16 \alpha(s-\lambda t)}{16 t \alpha^{2}} .
$$

As $m>n+p-2$, we have $\circ\left(r_{\infty}\right)=p+n-m<2$, which does not satisfy condition $(\infty)$ of case 3 of Kovacic's algorithm. Therefore $\operatorname{DGal}(\widehat{\mathbf{L}} / \widehat{K})$ is not finite primitive for any $\lambda \in \Lambda$.

Remark 6.19. In a natural way, we obtain the algebrized versions of Darboux transformation, i.e., the algebrized Darboux transformation, denoted by $\widehat{\mathrm{DT}}$. The notation $\widehat{\mathrm{DT}}_{n}$ stands for the $n$-th iteration of $\widehat{\mathrm{DT}}$; the notation $\widehat{\mathrm{CI}}_{n}$ denotes the $n$-th iteration of the algebrized Crum iteration, where the algebrized wronskian is given by

$$
\widehat{W}\left(\widehat{y}_{1}, \ldots, \widehat{y}_{n}\right)=\left|\begin{array}{ccc}
\widehat{y}_{1} & \ldots & \widehat{y}_{n} \\
\vdots & & \vdots \\
\widehat{\partial}_{z}^{n-1} \widehat{y}_{1} & \cdots & \widehat{\partial}_{z}^{n-1} \widehat{y}_{n}
\end{array}\right| .
$$

In the same way, we define algebrized shape invariant potentials, algebrized superpotential $\widehat{W}$, algebrized supersymmetric Hamiltonians $\widehat{H}_{ \pm}$, algebrized supersymmetric partner potentials $\widehat{V}_{ \pm}$, algebrized ground state $\widehat{\Psi}_{0}^{(-)}=e^{-\int \frac{\widehat{W}}{\sqrt{\alpha}} d z}$, algebrized wave functions $\widehat{\Psi}_{\lambda}^{(-)}$, algebrized raising and lowering operators $\widehat{A}$ and $\widehat{A}^{\dagger}$. Thus, we can rewrite entirely section 5 using the derivation $\widehat{\partial}_{z}$.

The following theorem shows us the relationship between the algebrization and the Darboux transformation.

Theorem 6.20. Given the Schrödinger equation $\mathcal{L}_{\lambda}:=H_{-} \Psi^{(-)}=\lambda \Psi^{(-)}$, the relationship between the algebrization $\varphi$ and Darboux transformations $\mathrm{DT}, \widehat{\mathrm{DT}}$ with respect to $\mathcal{L}_{\lambda}$ is given by $\widehat{\mathrm{DT}} \varphi=\varphi \mathrm{DT}$, that is $\widehat{\mathrm{DT}} \varphi(\mathcal{L})=\varphi \mathrm{DT}(\mathcal{L})$. In other words, the Darboux transformations DT and $\widehat{\mathrm{DT}}$ are intertwined by the algebrization $\varphi$.

Proof. Consider the equations $\mathcal{L}_{\lambda}:=H_{-} \Psi^{(-)}=\lambda \Psi^{(-)}, \widehat{\mathcal{L}}_{\lambda}:=\widehat{H}_{-} \widehat{\Psi}^{(-)}=$ $\lambda \widehat{\Psi}^{(-)}, \widetilde{\mathcal{L}}_{\lambda}:=H_{+} \Psi^{(+)}=\lambda \Psi^{(+)}$and $\widetilde{\widehat{\mathcal{L}}}:=\widehat{H}_{+} \widehat{\Psi}^{(+)}=\lambda \widehat{\Psi}^{(+)}$, where the Darboux transformations DT and $\widehat{\mathrm{DT}}$ are given by $\mathrm{DT}(\mathcal{L})=\widetilde{\mathcal{L}}, \widehat{\mathrm{DT}}(\widehat{\mathcal{L}})=\widetilde{\widehat{\mathcal{L}}}$,

$$
\begin{aligned}
& V_{-} \mapsto V_{+} \quad \widehat{V}_{-} \mapsto \widehat{V}_{+} \\
& \text {DT : } \widehat{\mathrm{DT}} \text { : } \\
& \Psi_{\lambda}^{(-)} \mapsto \Psi_{\lambda}^{(+)} \quad \widehat{\Psi}_{\lambda}^{(-)} \mapsto \widehat{\Psi}_{\lambda}^{(+)},
\end{aligned}
$$


and $\varphi\left(\mathcal{L}_{\lambda}\right)=\widehat{\mathcal{L}_{\lambda}}$, where the algebrization $\varphi$ is given as in theorem 6.13. Then the following diagram commutes

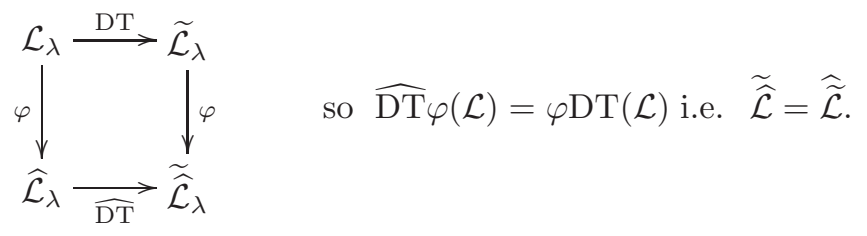

To illustrate $\widehat{\mathrm{DT}}$ we present the following examples.

Examples. Consider the algebrized Schrödinger equation $\widehat{H} \widehat{\Psi}^{(-)}=\lambda \widehat{\Psi}^{(-)}$with:

- $\sqrt{\alpha(z)}=\sqrt{z^{2}-1}$ and $\widehat{V}_{-}(z)=\frac{z}{z-1}$. Taking $\lambda_{1}=1$ and $\widehat{\Psi}_{1}^{(-)}=\sqrt{\frac{z+1}{z-1}}$, we have $\widehat{\mathrm{DT}}\left(\widehat{V}_{-}\right)=\widehat{V}_{+}(z)=\frac{z}{z+1}$ and

$$
\widehat{\mathrm{DT}}\left(\widehat{\Psi}_{\lambda}^{(-)}\right)=\widehat{\Psi}_{\lambda}^{(+)}=\sqrt{z^{2}-1} \partial_{z} \widehat{\Psi}_{\lambda}^{(-)}+\frac{1}{\sqrt{z^{2}-1}} \widehat{\Psi}_{\lambda}^{(-)},
$$

where $\widehat{\Psi}_{\lambda}^{(-)}$is the general solution of $\widehat{H}_{-} \widehat{\Psi}^{(-)}=\lambda \widehat{\Psi}^{(-)}$for $\lambda \neq 1$.

The original potential corresponding to this example is given by $V_{-}(x)=$ $\frac{\cosh x}{\cosh x-1}$ and for $\lambda_{1}=1$ the particular solution $\Psi_{1}^{(-)}$is given by $\frac{\sinh x}{\cosh x-1}$. Applying DT we have $\mathrm{DT}\left(V_{-}\right)=V_{+}(x)=\frac{\cosh x}{\cosh x+1}$ and $\operatorname{DT}\left(\Psi_{\lambda}^{(-)}\right)=$ $\Psi_{\lambda}^{(+)}=\partial_{x} \Psi_{\lambda}^{(-)}+\frac{1}{\sinh x} \Psi_{\lambda}^{(-)}$.

- $\sqrt{\alpha}=-z, \widehat{V}_{-}(z)=z^{2}-z$. Taking $\lambda_{1}=0$ and $\widehat{\Psi}_{0}^{(-)}=e^{-z}$ we have $\widehat{\mathrm{DT}}\left(\widehat{V}_{-}\right)=V_{+}=z^{2}+z$ and $\widehat{\mathrm{DT}}\left(\widehat{\Psi}_{\lambda}^{(-)}\right)=\widehat{\Psi}_{\lambda}^{(+)}=-z \partial_{z} \widehat{\Psi}_{\lambda}^{(-)}-z \widehat{\Psi}_{\lambda}^{(-)}$, where $\Psi_{\lambda}^{(-)}$is the general solution of $\widehat{H}_{-} \widehat{\Psi}^{(-)}=\lambda \widehat{\Psi}^{(-)}$for $\lambda \neq 0$. This example corresponds to the Morse potential $V_{-}(x)=e^{-2 x}-e^{-x}$.

To illustrate the algebrized Crum iteration $\widehat{\mathrm{CI}}_{n}$, we present the following example, which is related with the Chebyshev polynomials.

Example. Consider $\sqrt{\alpha}=-\sqrt{1-z^{2}}, V=0$ with eigenvalues and eigenfunctions $\lambda_{1}=1, \lambda_{2}=4, \widehat{\Psi}_{1}=z, \widehat{\Psi}_{4}=2 z^{2}-1, \widehat{\Psi}_{n^{2}}=T_{n}(z)$, where $T_{n}(z)$ is the Chebyshev polynomial of first kind of degree $n$. The algebrized Wronskian for $n=2$ is

$$
\widehat{W}\left(z, 2 z^{2}-1\right)=-\sqrt{1-z^{2}}\left(2 z^{2}+1\right),
$$

and by algebrized Crum iteration we obtain the potential

$$
\widehat{\mathrm{CI}}_{2}(\widehat{V})=\widehat{V}_{2}=\left(\left(2 z^{2}-1\right) \partial_{z}^{2}+z \partial_{z}\right) \ln \widehat{W}\left(z, 2 z^{2}-1\right)
$$

and the algebrized wave functions

$$
\widehat{\mathrm{CI}}_{2}\left(\widehat{\Psi}_{\lambda}\right)=\widehat{\Psi}_{\lambda}^{(2)}=\frac{\widehat{W}\left(z, 2 z^{2}-1, T_{n}\right)}{\widehat{W}\left(z, 2 z^{2}-1\right)} .
$$


In a natural way, we introduce the notion of algebrized shape invariant potentials $\widehat{V}_{n+1}\left(z, a_{n}\right)=\widehat{V}_{n}\left(z, a_{n+1}\right)+R\left(a_{n}\right)$, where the energy levels for $n>0$ are given by $E_{n}=R\left(a_{1}\right)+\cdots R\left(a_{n}\right)$ and the algebrized eigenfunctions are given by $\widehat{\Psi}_{n}\left(a_{1}\right)=\widehat{A}^{\dagger}\left(z, a_{1}\right) \cdots \widehat{A}^{\dagger}\left(z, a_{n}\right) \Psi_{0}\left(z, a_{n}\right)$. To illustrate the algebrized shape invariant potentials and the operators $\widehat{A}$ and $\widehat{A}^{\dagger}$, we present the following example.

Example. Let $\sqrt{\alpha}=1-z^{2}$ and consider the algebrized super potential $\widehat{W}(z)=z$. Following the method proposed in remark 4.10, step 1, we introduce $\mu \in \mathbb{C}$ to obtain $\widehat{W}(z ; \mu)=\mu z$, and

$$
\widehat{V}_{ \pm}(z ; \mu)=\widehat{W}^{2}(z ; \mu) \pm \widehat{\partial}_{z} \widehat{W}(z ; \mu)=\mu(\mu \mp 1) z^{2} \pm \mu .
$$

Thus $\widehat{V}_{+}\left(z ; a_{0}\right)=a_{0}\left(a_{0}-1\right) z^{2}+a_{0}$ and $\widehat{V}_{-}\left(z ; a_{1}\right)=a_{1}\left(a_{1}+1\right) z^{2}-a_{1}$. By step 2,

$$
\widehat{\partial}_{z}\left(V_{+}\left(z ; a_{0}\right)-V_{-}\left(z ; a_{1}\right)\right)=2 z\left(1-z^{2}\right)\left(a_{0}\left(a_{0}-1\right)-a_{1}\left(a_{1}+1\right)\right) .
$$

By step 3, we obtain

$$
a_{1}\left(a_{1}+1\right)=a_{0}\left(a_{0}-1\right), \quad a_{1}^{2}-a_{0}^{2}=-\left(a_{1}+a_{0}\right)
$$

and, assuming $a_{1} \neq \pm a_{0}$, we have $a_{1}=f\left(a_{0}\right)=a_{0}-1$ and $R\left(a_{1}\right)=2 a_{0}+1=$ $\left(a_{0}+1\right)^{2}-a_{0}^{2}=a_{1}^{2}-a_{0}^{2}$. This means that the potentials $\widehat{V}_{ \pm}$are algebrized shape invariant potentials where the $n$-th energy level $E=E_{n}$ is easily obtained:

$$
E_{n}=\sum_{k=1}^{n} R\left(a_{k}\right)=\sum_{k=1}^{n}\left(a_{k}^{2}-a_{k-1}^{2}\right)=a_{n}^{2}-a_{0}^{2}, \quad a_{n}=f^{n}\left(a_{0}\right)=a_{0}+n .
$$

Now, the algebrized ground state wave function of $\widehat{V}_{-}\left(z, a_{0}\right)$ is

$$
\widehat{\Psi}_{0}=e^{\int \frac{a_{0} z}{1-z^{2}} d z}=\frac{1}{\left(\sqrt{1-z^{2}}\right)^{a_{0}}} .
$$

Finally, we can obtain the rest of eigenfunctions using the algebrized raising operator:

$$
\widehat{\Psi}_{n}\left(z, a_{0}\right)=\widehat{A}^{\dagger}\left(z, a_{0}\right) \widehat{A}^{\dagger}\left(z, a_{1}\right) \cdots \widehat{A}^{\dagger}\left(z, a_{n-1}\right) \widehat{\Psi}_{0}\left(z, a_{n}\right) .
$$

This example corresponds to the Pöschl-Teller potential.

Now to illustrate the power of Kovacic's algorithm with the derivation $\widehat{\partial}_{z}$, we study some Schrödinger equations for non-rational shape invariant potentials.

Morse potential: $V(x)=e^{-2 x}-e^{-x}$.

The Schrödinger equation $H \Psi=\lambda \Psi$ is

$$
\partial_{x}^{2} \Psi=\left(e^{-2 x}-e^{-x}-\lambda\right) \Psi
$$

By the Hamiltonian change of variable $z=z(x)=e^{-x}$, we obtain

$$
\alpha(z)=z^{2}, \quad \widehat{V}(z)=z^{2}-z, \quad \widehat{\mathbf{V}}(z)=\frac{z^{2}-z-\frac{1}{4}}{z^{2}} .
$$

Thus, $\widehat{K}=\mathbb{C}(z)$ and $K=\mathbb{C}\left(e^{x}\right)$. The algebrized Schrödinger equation $\widehat{H} \widehat{\Psi}=\lambda \widehat{\Psi}$ is

$$
z^{2} \partial_{z}^{2} \widehat{\Psi}+z \partial_{z} \widehat{\Psi}-\left(z^{2}-z-\lambda\right) \widehat{\Psi}=0
$$


and the reduced algebrized Schrödinger equation $\widehat{\mathbf{H}} \widehat{\Phi}=\lambda \widehat{\Phi}$ is

$$
\partial_{z}^{2} \Phi=r \Phi, \quad r=\frac{z^{2}-z-\frac{1}{4}-\lambda}{z^{2}} .
$$

This equation could only fall under case 1, in case 2 or in case 4 (of Kovacic's algorithm). We start by analyzing the case 1: by conditions $c_{2}$ and $\infty_{3}$ we have that

$$
[\sqrt{r}]_{0}=0, \quad \alpha_{0}^{ \pm}=\frac{1 \pm 2 \sqrt{-\lambda}}{2}, \quad[\sqrt{r}]_{\infty}=1, \quad \text { and } \quad \alpha_{\infty}^{ \pm}=\mp \frac{1}{2} .
$$

By step 2 we have the following possibilities for $n \in \mathbb{Z}_{+}$and for $\lambda \in \Lambda$ :

$$
\begin{array}{lll}
\left.\Lambda_{++}\right) & n=\alpha_{\infty}^{+}-\alpha_{0}^{+}=-1-\sqrt{-\lambda}, & \lambda=-(n+1)^{2}, \\
\left.\Lambda_{+-}\right) & n=\alpha_{\infty}^{+}-\alpha_{0}^{-}=-1+\sqrt{-\lambda}, & \lambda=-(n+1)^{2}, \\
\left.\Lambda_{-+}\right) & n=\alpha_{\infty}^{-}-\alpha_{0}^{+}=-\sqrt{-\lambda}, & \lambda=-n^{2}, \\
\left.\Lambda_{--}\right) & n=\alpha_{\infty}^{-}-\alpha_{0}^{-}=\sqrt{-\lambda}, & \lambda=-n^{2} .
\end{array}
$$

We can see that $\lambda \in \Lambda_{-}=\left\{-n^{2}: n \in \mathbb{Z}_{+}\right\}$. Now, for $\lambda \in \Lambda$, the rational function $\omega$ is given by:

$$
\begin{array}{llll}
\left.\Lambda_{++}\right) & \omega=1+\frac{3+2 n}{2 z}, & \lambda \in \Lambda_{++}, & r_{n}=\frac{4 n^{2}+8 n+3}{4 z^{2}}+\frac{2 n+3}{z}+1, \\
\left.\Lambda_{+-}\right) & \omega=1-\frac{1+2 n}{2 z}, & \lambda \in \Lambda_{+-}, & r_{n}=\frac{4 n^{2}+8 n+3}{4 z^{2}}-\frac{2 n+1}{z}+1, \\
\left.\Lambda_{-+}\right) & \omega=-1+\frac{1+2 n}{2 z}, & \lambda \in \Lambda_{-+}, & r_{n}=\frac{4 n^{2}-1}{4 z^{2}}-\frac{2 n+1}{z}+1, \\
\left.\Lambda_{--}\right) & \omega=-1+\frac{1-2 n}{2 z}, & \lambda \in \Lambda_{--}, & r_{n}=\frac{4 n^{2}-1}{4 z^{2}}+\frac{2 n-1}{z}+1,
\end{array}
$$

where $r_{n}$ is the coefficient of the differential equation $\partial_{z}^{2} \Phi=r_{n} \Phi$.

By step 3, there exists a polynomial of degree $n$ satisfying the relation (A.1),

$$
\begin{array}{ll}
\left.\Lambda_{++}\right) & \partial_{z}^{2} \widehat{P}_{n}+2\left(1+\frac{3+2 n}{2 z}\right) \partial_{z} \widehat{P}_{n}+\frac{2(n+2)}{z} \widehat{P}_{n}=0 \\
\left.\Lambda_{+-}\right) & \partial_{z}^{2} \widehat{P}_{n}+2\left(1-\frac{1+2 n}{2 z}\right) \partial_{z} \widehat{P}_{n}+\frac{2(-n)}{z} \widehat{P}_{n}=0 \\
\left.\Lambda_{-+}\right) & \partial_{z}^{2} \widehat{P}_{n}+2\left(-1+\frac{1+2 n}{2 z}\right) \partial_{z} \widehat{P}_{n}+\frac{2(-n)}{z} \widehat{P}_{n}=0 \\
\left.\Lambda_{--}\right) & \partial_{z}^{2} \widehat{P}_{n}+2\left(-1+\frac{1-2 n}{2 z}\right) \partial_{z} \widehat{P}_{n}+\frac{2 n}{z} \widehat{P}_{n}=0
\end{array}
$$

These polynomials only exist for $n=\lambda=0$, with $\lambda \in \Lambda_{-+} \cup \Lambda_{--}$. So the solutions of $H \Psi=0, \widehat{H} \widehat{\Psi}=0$ and $\widehat{\mathbf{H}} \Phi=0$ are given by

$$
\Phi_{0}=\sqrt{z} e^{-z}, \quad \widehat{\Psi}_{0}=e^{-z}, \quad \Psi=e^{-e^{-x}} .
$$

The wave function $\Psi_{0}$ satisfy the bound state conditions, which means that is ground state (see [23]) and $0 \in \operatorname{Spec}_{p}(H)$. Furthermore, we have

$$
\operatorname{DGal}\left(L_{0} / K\right)=\operatorname{DGal}\left(\widehat{L}_{0} / \widehat{K}\right)=\operatorname{DGal}\left(\widehat{\mathbf{L}}_{0} / \mathbb{C}(z)\right)=\mathbb{B},
$$




$$
\mathcal{E}(H)=\mathcal{E}(\widehat{H})=\mathcal{E}(\widehat{\mathbf{H}})=\operatorname{Vect}(1)
$$

We proceed with case 2 . The conditions $c_{2}$ and $\infty_{3}$ are satisfied. So we have

$$
E_{c}=\{2,2+4 \sqrt{-\lambda}, 2-4 \sqrt{-\lambda}\} \quad \text { and } \quad E_{\infty}=\{0\},
$$

and by step two, we have that $2 \pm \sqrt{-\lambda}=m \in \mathbb{Z}_{+}$, so that $\lambda=-\left(\frac{m+1}{2}\right)^{2}$ and the rational function $\theta$ has the following possibilities

$$
\theta_{+}=\frac{2+m}{z}, \quad \theta_{-}=-\frac{m}{z} .
$$

By step three, there exist a monic polynomial of degree $m$ satisfying the recurrence relation A.2):

$$
\begin{array}{ll}
\left.\theta_{+}\right) \quad \partial_{z}^{3} \widehat{P}_{m}+\frac{3 m+6}{z} \partial_{z}^{2} \widehat{P}_{m}-\frac{4 z^{2}-4 z-2 m^{2}-7 m-6}{z^{2}} \partial_{z} \widehat{P}_{m}-\frac{4 m z+8 z-4 m-6}{z^{2}} \widehat{P}_{m} & =0 \\
\left.\theta_{-}\right) \quad \partial_{z}^{3} \widehat{P}_{m}-\frac{3 m}{z} \partial_{z}^{2} \widehat{P}_{m}-\frac{4 z^{2}-4 z-2 m^{2}-m}{z^{2}} \partial_{z} \widehat{P}_{m}+\frac{4 m z-4 m-2}{z^{2}} \widehat{P}_{m} & =0
\end{array}
$$

We can see that, for $m=1$, the polynomial exists only in the case $\theta_{-}$, with $\widehat{P}_{1}=z-1 / 2$. In general, these polynomials could exist only in the case $\theta_{-}$with $m=2 n-1, n \geq 1$, that is $\lambda \in\left\{-n^{2}: n \geq 1\right\}$.

By case one and case two, we obtain $\Lambda=\left\{-n^{2}: n \geq 0\right\}=\operatorname{Spec}_{p}(H)$. Now, the rational function $\phi$ and the quadratic expression for $\omega$ are

$$
\phi=-\frac{2 n-1}{z}+\frac{\partial_{z} \widehat{P}_{2 n-1}}{\widehat{P}_{2 n-1}}, \quad \omega^{2}+M \omega+N=0, \quad \omega=\frac{-M \pm \sqrt{M^{2}-4 N}}{2},
$$

where the coefficients $M$ and $N$ are given by

$M=\frac{2 n-1}{z}-\frac{\partial_{z} \widehat{P}_{2 n-1}}{\widehat{P}_{2 n-1}}, \quad N=\frac{n^{2}-n+\frac{1}{4}}{z^{2}}-\frac{(2 n-1) \frac{\partial_{z} \widehat{P}_{2 n-1}}{\widehat{P}_{2 n-1}}-2}{z}+\frac{\partial_{z}^{2} \widehat{P}_{2 n-1}}{\widehat{P}_{2 n-1}}-2$.

Now, $\triangle=M^{2}-4 N \neq 0$, which means that $\widehat{\mathbf{H}} \Phi=-n^{2} \Phi$ with $n \in \mathbb{Z}^{+}$has two solutions given by Kovacic's algorithm:

$$
\Phi_{1, n}=\frac{\sqrt{z} \widehat{P}_{n} e^{-z}}{z^{n}}, \quad \Phi_{2, n}=\frac{\sqrt{z} \widehat{P}_{n-1} e^{z}}{z^{n}} .
$$

The solutions of $\widehat{H} \widehat{\Psi}=-n^{2} \widehat{\Psi}$ are given by

$$
\widehat{\Psi}_{1, n}=\frac{\widehat{P}_{n} e^{-z}}{z^{n}}, \quad \widehat{\Psi}_{2, n}=\frac{\widehat{P}_{n-1} e^{z}}{z^{n}},
$$

and therefore, the solutions of the Schrödinger equation $H \Psi=-n^{2} \Psi$ are

$$
\Psi_{1, n}=P_{n} e^{-e^{-x}} e^{n x}, \quad \Psi_{2, n}=P_{n-1} e^{e^{-x}} e^{n x}, \quad P_{n}=\widehat{P}_{n} \circ z .
$$

The wave functions $\Psi_{1, n}=\Psi_{n}$ satisfy the conditions of bound state and, for $n=0$, this solution coincides with the ground state presented above. Therefore we have

$$
\Phi_{n}=\Phi_{0} \widehat{f}_{n} \widehat{P}_{n}, \quad \widehat{\Psi}_{n}=\widehat{\Psi}_{0} \widehat{f}_{n} \widehat{P}_{n}, \quad \widehat{f}_{n}(z)=\frac{1}{z^{n}} .
$$

Thus, the bound states wave functions are obtained as

$$
\Psi_{n}=\Psi_{0} f_{n} P_{n}, \quad f_{n}(x)=\widehat{f}_{n}\left(e^{-x}\right)=e^{n x} .
$$


The eigenring s and differential Galois groups for $n>0$ satisfies

$$
\begin{gathered}
\operatorname{DGal}\left(L_{n} / K\right)=\operatorname{DGal}\left(\widehat{L}_{n} / \widehat{K}\right)=\operatorname{DGal}\left(\widehat{\mathbf{L}}_{n} / \mathbb{C}(z)\right)=\mathbb{G}_{m}, \\
\operatorname{dim}_{\mathbb{C}} \mathcal{E}\left(\widehat{\mathbf{H}}+n^{2}\right)=\operatorname{dim}_{\mathbb{C}} \mathcal{E}\left(\widehat{H}+n^{2}\right)=\operatorname{dim}_{\mathbb{C}} \mathcal{E}\left(H+n^{2}\right)=2 .
\end{gathered}
$$

We remark that the Schrödinger equation with Morse potential, under suitable changes of variables [38, may be transformed to a Bessel differential equation.

It is known that equations deriving from Eckart, Rosen-Morse, Scarf and PöschlTeller potentials may be mapped, under suitable transformations, to Hypergeometric equations. These potentials are inter-related by point canonical coordinate transformations (see [16 p. 314]), so that $\Lambda=\mathbb{C}$ because Pöschl-Teller potential is obtained by means of Darboux transformations of $V=0([\mathbf{4 2}, \mathbf{5 2}])$. We consider some particular cases of Eckart, Scarf and Poschl-Teller potentials for which we apply only case 1 of Kovacic's algorithm. Case 1 allows us to obtain the enumerable set $\Lambda_{n} \subset \Lambda$, which includes the classical results obtained by means of supersymmetric quantum mechanics. Cases 2 and 3 of Kovacic algorithm also can be applied, but are not considered here.

Eckart potential: $V(x)=4 \operatorname{coth}(x)+5, x>0$.

The Schrödinger equation $H \Psi=\lambda \Psi$ is

$$
\partial_{x}^{2} \Psi=(4 \operatorname{coth}(x)+5-\lambda) \Psi .
$$

By the Hamiltonian change of variable $z=z(x)=\operatorname{coth}(x)$, we obtain

$$
\alpha(z)=\left(1-z^{2}\right)^{2}, \quad \widehat{V}(z)=4 z+5, \quad \widehat{\mathbf{V}}(z)=\frac{4}{(z+1)(z-1)^{2}} .
$$

Thus, $\widehat{K}=\mathbb{C}(z)$ and $K=\mathbb{C}(\operatorname{coth}(x))$. The algebrized Schrödinger equation $\widehat{H} \widehat{\Psi}=$ $\lambda \widehat{\Psi}$ is

$$
\left(1-z^{2}\right)^{2} \partial_{z}^{2} \widehat{\Psi}-2 z\left(1-z^{2}\right) \partial_{z} \widehat{\Psi}-(4 z+5-\lambda) \widehat{\Psi}=0
$$

and the reduced algebrized Schrödinger equation $\widehat{\mathbf{H}} \widehat{\Phi}=\lambda \widehat{\Phi}$ is

$$
\partial_{z}^{2} \Phi=r \Phi, \quad r=\frac{4 z+4-\lambda}{(z-1)^{2}(z+1)^{2}}=\frac{2-\frac{\lambda}{4}}{(z-1)^{2}}+\frac{\frac{\lambda}{4}-1}{(z-1)}+\frac{-\frac{\lambda}{4}}{(z+1)^{2}}+\frac{1-\frac{\lambda}{4}}{(z+1)}
$$

We can see that this equation could fall in any case of Kovacic's algorithm. Considering $\lambda=0$, the conditions $\left\{c_{1}, c_{2}, \infty_{1}\right\}$ of case 1 are satisfied, obtaining

$$
[\sqrt{r}]_{-1}=[\sqrt{r}]_{1}=[\sqrt{r}]_{\infty}=\alpha_{\infty}^{+}=0, \quad \alpha_{-1}^{ \pm}=\alpha_{\infty}^{-}=1, \quad \alpha_{1}^{+}=2, \quad \alpha_{1}^{-}=-1 .
$$

By step 2, the elements of $D$ are 0 and 1 . The rational function $\omega$ for $n=0$ and for $n=1$ must be

$$
\omega=\frac{1}{z+1}+\frac{-1}{z-1} .
$$

By step 3, we search for a monic polynomial of degree $n$ satisfying the relation (A.1). Starting with $n=0$ the only one possibility is $\widehat{P}_{0}(z)=1$, which effectively satisfy the relation (A.1), while $\widehat{P}_{1}(z)=z+a_{0}$ does not exist. We have obtained one solution using Kovacic algorithm:

$$
\Phi_{0}=\frac{z+1}{z-1}, \quad \widehat{\Psi}_{0}=\sqrt{\frac{z+1}{(z-1)^{3}}},
$$


this means that $0 \in \Lambda_{n}$. We can obtain the second solution using the first solution:

$$
\Phi_{0,2}=\frac{z^{2}+z-4-4 \ln (z+1) z-4 \ln (z+1)}{z-1}, \quad \widehat{\Psi}_{0,2}=\frac{\Phi_{0,2}}{\sqrt{z^{2}-1}} .
$$

Furthermore the differential Galois groups and eigenring s for $\lambda=0$ are

$$
\begin{gathered}
\operatorname{DGal}\left(\widehat{\mathbf{L}}_{0} / \mathbb{C}(z)\right)=\mathbb{G}_{a}, \quad \operatorname{DGal}\left(L_{0} / K\right)=\operatorname{DGal}\left(\widehat{L}_{0} / \widehat{K}\right)=\mathbb{G}^{\{2\}}, \\
\mathcal{E}(\widehat{\mathbf{H}})=\operatorname{Vect}\left(1, \frac{(z+1)^{2}}{(1-z)^{2}} \partial_{z}+\frac{2(z+1)}{(1-z)^{3}}\right), \\
\mathcal{E}(\widehat{H})=\operatorname{Vect}\left(1, \frac{(z+1)^{2}}{(1-z)^{2}} \partial_{z}-\frac{z^{2}+3 z+2}{(1-z)^{3}}\right), \\
\mathcal{E}(H)=\operatorname{Vect}\left(1, \frac{(\operatorname{coth}(x)+1)^{2}}{(1-\operatorname{coth}(x))^{2}\left(1-\operatorname{coth}^{2}(x)\right)} \partial_{x}-\frac{\operatorname{coth}^{2}(x)+3 \operatorname{coth}(x)+2}{(1-\operatorname{coth}(x))^{3}}\right) .
\end{gathered}
$$

Now, for $\lambda \neq 0$, the conditions $\left\{c_{2}, \infty_{1}\right\}$ of case 1 are satisfied:

$$
\begin{aligned}
& {[\sqrt{r}]_{-1}=[\sqrt{r}]_{1}=[\sqrt{r}]_{\infty}=\alpha_{\infty}^{+}=0, \quad \alpha_{\infty}^{-}=1,} \\
& \alpha_{-1}^{ \pm}=\frac{1 \pm \sqrt{1-\lambda}}{2}, \quad \alpha_{1}^{ \pm}=\frac{1 \pm \sqrt{9-\lambda}}{2} .
\end{aligned}
$$

By step 2 we have the following possibilities for $n \in \mathbb{Z}_{+}$and for $\lambda \in \Lambda$ :

$$
\begin{array}{lll}
\left.\Lambda_{++-}\right) & n=\alpha_{\infty}^{+}-\alpha_{-1}^{+}-\alpha_{1}^{-}=-1-\frac{\sqrt{1-\lambda}-\sqrt{9-\lambda}}{2}, & \lambda=4-\frac{4}{(n+1)^{2}}-n^{2}-2 n, \\
\left.\Lambda_{+--}\right) & n=\alpha_{\infty}^{+}-\alpha_{-1}^{-}-\alpha_{1}^{-}=-1+\frac{\sqrt{1-\lambda}+\sqrt{9-\lambda}}{2}, & \lambda=4-\frac{4}{(n+1)^{2}}-n^{2}-2 n, \\
\left.\Lambda_{-+-}\right) & n=\alpha_{\infty}^{-}-\alpha_{-1}^{+}-\alpha_{1}^{-}=\frac{\sqrt{1-\lambda}+\sqrt{9-\lambda}}{2}, & \lambda=5-\frac{4}{n^{2}}-n^{2}, \\
\left.\Lambda_{---}\right) & n=\alpha_{\infty}^{-}-\alpha_{-1}^{-}-\alpha_{1}^{-}=\frac{\sqrt{1-\lambda}+\sqrt{9-\lambda}}{2}, & \lambda=5-\frac{4}{n^{2}}-n^{2} .
\end{array}
$$

Therefore, we have

$$
\Lambda_{n} \subseteq\left\{4-\frac{4}{(n+1)^{2}}-n^{2}-2 n: n \in \mathbb{Z}_{+}\right\} \cup\left\{5-\frac{4}{n^{2}}-n^{2}: n \in \mathbb{Z}_{+}\right\} .
$$

Now, for $\lambda \in \Lambda$, the rational function $\omega$ is given by:

$$
\begin{array}{llll}
\left.\Lambda_{++-}\right) & \omega=\frac{z(n-1)-n^{2}-2 n-1}{(n+1)(z+1)(z-1)}, & r_{n}=\frac{-2 z^{2}(n-1)+4 z(n+1)^{2}+(n+1)\left(n^{3}+3 n^{2}+2 n+2\right)}{(n+1)^{2}(z+1)^{2}(z-1)^{2}}, \\
\left.\Lambda_{+--}\right) & \omega=\frac{n z(n+1)+2}{(n+1)(z+1)(1-z)}, & r_{n}=\frac{n z^{2}(n+1)^{3}+4 z(n+1)^{2}+n^{3}+2 n^{2}+n+4}{(n+1)^{2}(z+1)^{2}(z-1)^{2}}, \\
\left.\Lambda_{-+-)}\right) & \omega=\frac{z(n-2)-n^{2}}{n(z+1)(z-1)}, & r_{n}=\frac{-2 z^{2}(n-2)+4 n^{2} z+n\left(n^{3}-n+2\right)}{n^{2}(z+1)^{2}(z-1)^{2}}, \\
\left.\Lambda_{---}\right) & \omega=\frac{n z(n-1)+2}{n(z+1)(1-z)}, & r_{n}=\frac{n^{3} z^{2}(n-1)+4 n^{2} z+n^{3}-n^{2}+4}{n^{2}(z+1)^{2}(z-1)^{2}},
\end{array}
$$

where $r_{n}$ is the coefficient of the differential equation $\partial_{z}^{2} \Phi=r_{n} \Phi$. 
By step 3, there exists a monic polynomial of degree $n$ satisfying the relation (A.1),

$$
\begin{array}{lll}
\left.\Lambda_{++-}\right) & \partial_{z}^{2} \widehat{P}_{n}+2\left(\frac{z(n-1)-n^{2}-2 n-1}{(n+1)(z+1)(z-1)}\right) \partial_{z} \widehat{P}_{n}+\frac{2(1-n)}{\left((n+1)^{2}(z+1)(z-1)\right.} \widehat{P}_{n} & =0, \\
\left.\Lambda_{+--}\right) & \partial_{z}^{2} \widehat{P}_{n}+2\left(\frac{n z(n+1)+2}{(n+1)(z+1)(1-z)}\right) \partial_{z} \widehat{P}_{n}+\frac{n(n+1)}{(z+1)(z-1)} \widehat{P}_{n} & =0, \\
\left.\Lambda_{-+-}\right) & \partial_{z}^{2} \widehat{P}_{n}+2\left(\frac{z(n-2)-n^{2}}{n(z+1)(z-1)}\right) \partial_{z} \widehat{P}_{n}+\frac{2(2-n)}{n^{2}(z+1)(z-1)} \widehat{P}_{n} & =0, \\
\left.\Lambda_{---}\right) & \partial_{z}^{2} \widehat{P}_{n}+2\left(\frac{n z(n-1)+2}{n(z+1)(1-z)}\right) \partial_{z} \widehat{P}_{n}+\frac{n(n-1)}{(z+1)(z-1)} \widehat{P}_{n} & =0 .
\end{array}
$$

The only one case in which there exists such a polynomial $\widehat{P}_{n}$ of degree $n$ is for $\left.\Lambda_{+--}\right)$. The solutions of the equation $\widehat{\mathbf{H}} \Phi=\lambda \Phi$, with $\lambda \neq 0$, are:

$$
\begin{aligned}
& \left.\Lambda_{++-}\right) \quad \Phi_{n}=\widehat{P}_{n} \widehat{f}_{n} \Phi_{0}, \quad \Phi_{0}=\frac{1}{z-1} \quad \widehat{f}_{n}=(z-1)^{\frac{n(1-n)}{2(n+1)}}(z+1)^{\frac{n(n+3)}{2(n+1)}}, \\
& \left.\Lambda_{+--}\right) \quad \Phi_{n}=\widehat{P}_{n} \widehat{f}_{n} \Phi_{0}, \quad \Phi_{0}=\frac{z+1}{z-1} \quad f_{n}=(z-1)^{\frac{n(1-n)}{2(n+1)}}(z+1)^{\frac{-n(n+3)}{2(n+1)}}, \\
& \left.\Lambda_{-+-}\right) \quad \Phi_{n}=\widehat{P}_{n} \widehat{f}_{n} \Phi_{1}, \quad \Phi_{1}=\frac{1}{z-1} \quad \widehat{f}_{n}=(z+1)^{\frac{n^{2}+n-2}{2 n}}(z-1)^{\frac{-n^{2}+3 n-2}{2 n}}, \\
& \left.\Lambda_{---}\right) \quad \Phi_{n}=\widehat{P}_{n} \widehat{f}_{n} \Phi_{1}, \quad \Phi_{1}=\frac{z+1}{z-1} \quad \widehat{f}_{n}=(z+1)^{\frac{-n^{2}-n+2}{2 n}}(z-1)^{\frac{-n^{2}+3 n-2}{2 n}} .
\end{aligned}
$$

In any case $\widehat{\Psi}_{n}=\frac{\Phi_{n}}{1-z^{2}}$. The case $\Lambda_{+--}$) includes the classical results obtained by means of supersymmetric quantum mechanics. Thus, replacing $z$ by $\operatorname{coth}(x)$ we obtain the eigenstates $\Psi_{n}$. The eigenring s and differential Galois groups for $n>0$ and $\lambda \in \Lambda_{n}$ satisfy

$$
\begin{gathered}
\operatorname{DGal}\left(L_{\lambda} / K\right) \subseteq \mathbb{G}^{\{2 m\}}, \quad \operatorname{DGal}\left(\widehat{L}_{\lambda} / \widehat{K}\right) \subseteq \mathbb{G}^{\{2 m\}}, \\
\operatorname{DGal}\left(\widehat{\mathbf{L}}_{\lambda} / \mathbb{C}(z)\right)=\mathbb{G}_{m}, \\
\operatorname{dim}_{\mathbb{C}(z)} \mathcal{E}(\widehat{\mathbf{H}}+\lambda)=2, \quad \mathcal{E}(\widehat{H}+\lambda)=\mathcal{E}(H+\lambda)=\operatorname{Vect}(1) .
\end{gathered}
$$

Scarf potential: $V(x)=\frac{\sinh ^{2} x-3 \sinh x}{\cosh ^{2} x}$.

The Schrödinger equation $H \Psi=E \Psi$ is

$$
\partial_{x}^{2} \Psi=\left(\frac{\sinh ^{2} x-3 \sinh x}{\cosh ^{2} x}-E\right) \Psi .
$$

By the Hamiltonian change of variable $z=z(x)=\sinh (x)$, we obtain

$$
\alpha(z)=1+z^{2}, \quad \widehat{V}(z)=\frac{z^{2}-3 z}{1+z^{2}} .
$$

Thus, $\widehat{K}=\mathbb{C}\left(z, \sqrt{1+z^{2}}\right)$ and $K=\mathbb{C}(\sinh (x), \cosh (x))$. The reduced algebrized Schrödinger equation $\widehat{\mathbf{H}} \Phi=\lambda \Phi$ is

$$
\partial_{z}^{2}=\left(\frac{\lambda z^{2}-12 z+\lambda-1}{4\left(z^{2}+1\right)^{2}}\right) \Phi, \quad \lambda=3-4 E
$$


Applying Kovacic's algorithm for this equation with $\lambda=0$, we see that it does not satisfy case 1 . So we consider only $\lambda \neq 0$. By conditions $\left\{c_{2}, \infty_{2}\right\}$ of case 1 we have

$$
\begin{aligned}
& {[\sqrt{r}]_{-\mathrm{i}}=[\sqrt{r}]_{\mathrm{i}}=[\sqrt{r}]_{\infty}=0, \quad \alpha_{\infty}^{ \pm}=\frac{1 \pm \sqrt{1+\lambda}}{2},} \\
& \alpha_{-\mathrm{i}}^{+}=\frac{5}{4}-\frac{\mathrm{i}}{2}, \quad \alpha_{-\mathrm{i}}^{-}=-\frac{1}{4}+\frac{\mathrm{i}}{2} \quad \alpha_{\mathrm{i}}^{+}=\frac{5}{4}+\frac{\mathrm{i}}{2}, \quad \alpha_{\mathrm{i}}^{-}=-\frac{1}{4}-\frac{\mathrm{i}}{2} .
\end{aligned}
$$

By step 2 we have the following possibilities for $n \in \mathbb{Z}_{+}$and for $\lambda \in \Lambda$ :

$$
\begin{array}{lll}
\left.\Lambda_{+++}\right) & n=\alpha_{\infty}^{+}-\alpha_{-\mathrm{i}}^{+}-\alpha_{\mathrm{i}}^{+}=\frac{\sqrt{\lambda+1}-4}{2}, & \lambda=4 n^{2}+16 n+15, \\
\left.\Lambda_{+--}\right) & n=\alpha_{\infty}^{+}-\alpha_{-\mathrm{i}}^{-}-\alpha_{\mathrm{i}}^{-}=\frac{\sqrt{\lambda+1}+2}{2}, & \lambda=4 n^{2}-8 n+3,
\end{array}
$$

thus obtaining

$$
\Lambda_{n} \subseteq\left\{4 n^{2}+16 n+15: n \in \mathbb{Z}_{+}\right\} \cup\left\{4 n^{2}-8 n+3: n \in \mathbb{Z}_{+}\right\} .
$$

Now, the rational function $\omega$ is given by:

$$
\left.\left.\Lambda_{+++}\right) \quad \omega=\frac{5 z-2}{2\left(z^{2}+1\right)}, \quad \Lambda_{+--}\right) \quad \frac{2-z}{2\left(z^{2}+1\right)} .
$$

By step 3 , there exists $\widehat{P}_{0}=1$ and a polynomial of degree $n \geq 1$ should satisfy either of the relation A.1,

$$
\begin{array}{ll}
\left.\Lambda_{+++}\right) & \partial_{z}^{2} \widehat{P}_{n}+\frac{5 z-2}{z^{2}+1} \partial_{z} \widehat{P}_{n}-\frac{n z^{2}(n+4)+3 z+n^{2}+4 n-3}{\left(z^{2}+1\right)^{2}} \widehat{P}_{n}=0, \\
\left.\Lambda_{+--}\right) & \partial_{z}^{2} \widehat{P}_{n}+\frac{2-z}{z^{2}+1} \partial_{z} \widehat{P}_{n}-\frac{n z^{2}(n-2)+3 z+n^{2}-2 n-3}{\left(z^{2}+1\right)^{2}} \widehat{P}_{n}=0 .
\end{array}
$$

In both cases there exists such a polynomial $\widehat{P}_{n}$ of degree $n \geq 1$. Basis of solutions $\left\{\Phi_{1, n}, \Phi_{2, n}\right\}$ of the reduced algebrized Schrödinger equation are:

$$
\begin{array}{rlrl}
\left.\Lambda_{+++}\right) & \Phi_{1, n}=\widehat{P}_{n} \widehat{f}_{n} \Phi_{1,0}, & \Phi_{1,0}=\left(1+z^{2}\right)^{\frac{5}{4}} e^{-\arctan z}, & \widehat{f}_{n}=1, \\
\Phi_{2, n}=\widehat{Q}_{n} \widehat{g}_{n} \Phi_{2,0}, & \Phi_{2,0}=\frac{22+21 x+12 x^{2}+6 x^{3}}{\sqrt[4]{1+z^{2}}} e^{-\arctan z}, & \widehat{g}_{n}=1 . \\
\left.\Lambda_{+--}\right) & \Phi_{1, n}=\widehat{P}_{n} \widehat{f}_{n} \Phi_{1,0}, & \Phi_{1,0}=\frac{1}{\sqrt[4]{1+z^{2}}} e^{\arctan z}, & \widehat{f}_{n}=1, \\
\Phi_{2, n}=\widehat{Q}_{n} \widehat{g}_{n} \Phi_{2,0}, & \Phi_{2,0}=\frac{1}{\sqrt[4]{1+z^{2}}} e^{\arctan z} \int \frac{1}{\sqrt{1+z^{2}}} e^{-2 \arctan z} d z, & \widehat{g}_{n}=1 .
\end{array}
$$

In both cases $\widehat{\Psi}=\frac{\Phi}{\sqrt[4]{1+z^{2}}}$, but the classical case (see references [16, 23]) is $\Lambda_{+--}$), so that replacing $z$ by $\sinh x$ and $\lambda$ by $3-4 E$ we obtain the eigenstates $\Psi_{n}$.

The eigenring s and differential Galois groups are

$$
\begin{aligned}
\mathcal{E}(H-\lambda) & =\mathcal{E}(\widehat{H}-\lambda)=\mathcal{E}(\widehat{\mathbf{H}}-\lambda)=\operatorname{Vect}(1), \\
\operatorname{DGal}\left(L_{\lambda} / K\right) & =\operatorname{DGal}\left(\widehat{L}_{\lambda} / \widehat{K}\right)=\operatorname{DGal}\left(\widehat{\mathbf{L}}_{\lambda} / \mathbb{C}(x)\right)=\mathbb{B} .
\end{aligned}
$$


Pöschl-Teller potential (revisited): $V(\mathrm{r})=\frac{\cosh ^{4}(x)-\cosh ^{2}(x)+2}{\sinh ^{2}(x) \cosh ^{2}(x)}, x>0$. The reduced algebrized Schrödinger equation $\widehat{\mathbf{H}} \Phi=E \Phi$ is

$$
\partial_{z}^{2} \Phi=\left(\frac{\lambda z^{4}-(\lambda+3) z^{2}+8}{4 z^{2}\left(z^{2}-1\right)^{2}}\right) \Phi, \quad \lambda=3-4 E
$$

Considering $\lambda=0$ and starting with the conditions $\left\{c_{2}, \infty_{1}\right\}$ of case 1 , we obtain

$$
\begin{aligned}
& {[\sqrt{r}]_{0}=[\sqrt{r}]_{-1}=[\sqrt{r}]_{1}=[\sqrt{r}]_{\infty}=\alpha_{\infty}^{+}=0, \quad \alpha_{\infty}^{-}=1,} \\
& \alpha_{-1}^{+}=\alpha_{1}^{+}=\frac{5}{4}, \quad \alpha_{-1}^{-}=\alpha_{1}^{-}=-\frac{1}{4}, \quad \alpha_{0}^{+}=2, \quad \alpha_{0}^{-}=-1 .
\end{aligned}
$$

By step 2, the elements of $D$ are 0 and 1 . For the rational function $\omega$, we have the following possibilities for $n=0$ and for $n=1$ :

$$
\begin{aligned}
& \left.\Lambda_{++--}\right) \quad n=0, \quad \omega=\frac{5 / 4}{z+1}+\frac{-1 / 4}{z-1}+\frac{-1}{z} \\
& \left.\Lambda_{+-+-}\right) \quad n=0, \quad \omega=\frac{-1 / 4}{z+1}+\frac{5 / 4}{z-1}+\frac{-1}{z} \\
& \left.\Lambda_{-+--}\right) \quad n=1, \quad \omega=\frac{5 / 4}{z+1}+\frac{-1 / 4}{z-1}+\frac{-1}{z} \\
& \left.\Lambda_{--+-}\right) \quad n=1, \quad \omega=\frac{-1 / 4}{z+1}+\frac{5 / 4}{z-1}+\frac{-1}{z} .
\end{aligned}
$$

Following step 3, we search for a monic polynomial of degree $n$ satisfying relation (A.1). Starting with $n=0$ the only possibility for $\left.\Lambda_{++--}\right)$and $\left.\Lambda_{+-+-}\right)$is $\widehat{P}_{0}(z)=$ 1, which does not satisfy relation (A.1) in both cases, while $\widehat{P}_{1}(z)=z+a_{0}$ effectively does exist, taking $a_{0}=-\frac{2}{3}$ for $\Lambda_{-+--}$) and $a_{0}=\frac{2}{3}$ for $\left.\Lambda_{--+-}\right)$. This way, we have obtained two solutions $\left(\Phi_{1,0}, \Phi_{2,0}\right)$ using Kovacic's algorithm:

$$
\begin{array}{ll}
\Phi_{1,0}=\left(1-\frac{2}{3 z}\right) \sqrt[4]{\frac{(z+1)^{5}}{z-1}}, & \widehat{\Psi}_{1,0}=\left(1-\frac{2}{3 z}\right) \frac{z+1}{\sqrt{z-1}} \\
\Phi_{2,0}=\left(1+\frac{2}{3 z}\right) \sqrt[4]{\frac{(z-1)^{5}}{z+1}}, & \widehat{\Psi}_{2,0}=\left(1+\frac{2}{3 z}\right) \frac{z-1}{\sqrt{z+1}}
\end{array}
$$

This means that $0 \in \Lambda_{n}$. Furthermore,

$$
\begin{gathered}
\operatorname{DGal}\left(\widehat{\mathbf{L}}_{0} / \mathbb{C}(x)\right)=\mathbb{G}^{[4]}, \quad \operatorname{DGal}\left(\widehat{L}_{0} / \widehat{K}\right)=\operatorname{DGal}\left(L_{0} / K\right)=e, \\
\operatorname{dim}_{\mathbb{C}} \mathcal{E}(\widehat{\mathbf{H}})=2, \quad \operatorname{dim}_{\mathbb{C}} \mathcal{E}(\widehat{H})=\operatorname{dim}_{\mathbb{C}} \mathcal{E}(H)=4 .
\end{gathered}
$$

Now, for $\lambda \neq 0$, we see that conditions $\left\{c_{2}, \infty_{1}\right\}$ of case 1 leads us to

$$
\begin{aligned}
& {[\sqrt{r}]_{0}=[\sqrt{r}]_{-1}=[\sqrt{r}]_{1}=[\sqrt{r}]_{\infty}=0, \quad \alpha_{\infty}^{ \pm}=\frac{1 \pm \sqrt{1+\lambda}}{2},} \\
& \alpha_{-1}^{+}=\alpha_{1}^{+}=\frac{5}{4}, \quad \alpha_{-1}^{-}=\alpha_{1}^{-}=-\frac{1}{4}, \quad \alpha_{0}^{+}=2, \quad \alpha_{0}^{-}=-1 .
\end{aligned}
$$


By step 2 we have the following possibilities for $n \in \mathbb{Z}_{+}$and for $\lambda \in \Lambda$ :

$$
\begin{array}{lll}
\left.\Lambda_{++++}\right) & n=\alpha_{\infty}^{+}-\alpha_{-1}^{+}-\alpha_{1}^{+}-\alpha_{0}^{+}=\frac{\sqrt{\lambda+1}-8}{2}, \quad \lambda=4 n^{2}+32 n+63, \\
\left.\Lambda_{+++-}\right) & n=\alpha_{\infty}^{+}-\alpha_{-1}^{+}-\alpha_{1}^{+}-\alpha_{0}^{-}=\frac{\sqrt{\lambda+1}-2}{2}, \quad \lambda=4 n^{2}+8 n+3, \\
\left.\Lambda_{++-+}\right) & n=\alpha_{\infty}^{+}-\alpha_{-1}^{+}-\alpha_{1}^{-}-\alpha_{0}^{+}=\frac{\sqrt{\lambda+1}-5}{2}, \quad \lambda=4 n^{2}+20 n+24, \\
\left.\Lambda_{++--}\right) & n=\alpha_{\infty}^{+}-\alpha_{-1}^{+}-\alpha_{1}^{-}-\alpha_{0}^{-}=\frac{\sqrt{\lambda+1}+1}{2}, \quad \lambda=4 n^{2}-4 n, \\
\left.\Lambda_{+-++}\right) \quad n=\alpha_{\infty}^{+}-\alpha_{-1}^{-}-\alpha_{1}^{+}-\alpha_{0}^{+}=\frac{\sqrt{\lambda+1}-5}{2}, & \lambda=4 n^{2}+20 n+24, \\
\left.\Lambda_{+-+-}\right) \quad n=\alpha_{\infty}^{+}-\alpha_{-1}^{-}-\alpha_{1}^{+}-\alpha_{0}^{-}=\frac{\sqrt{\lambda+1}+1}{2}, & \lambda=4 n^{2}-4 n, \\
\left.\Lambda_{+--+}\right) \quad n=\alpha_{\infty}^{+}-\alpha_{-1}^{-}-\alpha_{1}^{-}-\alpha_{0}^{+}=\frac{\sqrt{\lambda+1}-2}{2}, & \lambda=4 n^{2}+8 n+3, \\
\left.\Lambda_{+---}\right) & n=\alpha_{\infty}^{+}-\alpha_{-1}^{-}-\alpha_{1}^{-}-\alpha_{0}^{-}=\frac{\sqrt{\lambda+1}+4}{2}, & \lambda=4 n^{2}-16 n+15,
\end{array}
$$

obtaining $\Lambda_{n} \subseteq \Lambda_{a} \cup \Lambda_{b} \cup \Lambda_{c} \cup \Lambda_{d} \cup \Lambda_{e}$, where

$$
\begin{array}{ll}
\Lambda_{a}=\left\{4 n^{2}+32 n+63: n \in \mathbb{Z}_{+}\right\}, & \Lambda_{b}=\left\{4 n^{2}+8 n+3: n \in \mathbb{Z}_{+}\right\} \\
\Lambda_{c}=\left\{4 n^{2}+20 n+24: n \in \mathbb{Z}_{+}\right\}, & \Lambda_{d}=\left\{4 n^{2}-4 n: n \in \mathbb{Z}_{+}\right\}, \\
\Lambda_{e}=\left\{4 n^{2}-16 n+15: n \in \mathbb{Z}_{+}\right\} . &
\end{array}
$$

Now, the rational function $\omega$ is given by, respectively:

$$
\begin{array}{llll}
\left.\Lambda_{++++}\right) & \omega=\frac{5 / 4}{z+1}+\frac{5 / 4}{z-1}+\frac{2}{z}, & \left.\Lambda_{+++-}\right) & \omega=\frac{5 / 4}{z+1}+\frac{5 / 4}{z-1}+\frac{-1}{z}, \\
\left.\Lambda_{++-+}\right) & \omega=\frac{5 / 4}{z+1}+\frac{-1 / 4}{z-1}+\frac{2}{z}, & \left.\Lambda_{++--}\right) & \omega=\frac{5 / 4}{z+1}+\frac{-1 / 4}{z-1}+\frac{-1}{z}, \\
\left.\Lambda_{+-++}\right) & \omega=\frac{-1 / 4}{z+1}+\frac{5 / 4}{z-1}+\frac{2}{z}, & \left.\Lambda_{+-+-}\right) & \omega=\frac{-1 / 4}{z+1}+\frac{5 / 4}{z-1}+\frac{-1}{z}, \\
\left.\Lambda_{+--+}\right) & \omega=\frac{-1 / 4}{z+1}+\frac{-1 / 4}{z-1}+\frac{2}{z}, & \left.\Lambda_{+---}\right) & \omega=\frac{-1 / 4}{z+1}+\frac{-1 / 4}{z-1}+\frac{-1}{z} .
\end{array}
$$


By step 3, there exists a monic polynomial of degree $n$ satisfying relation (A.1):

$$
\begin{array}{ll}
\left.\Lambda_{++++}\right) & \partial_{z}^{2} \widehat{P}_{n}+\frac{(3 z+2)(3 z-2)}{z(z+1)(z-1)} \partial_{z} \widehat{P}_{n}+\frac{n(n+8)}{(z+1)(1-z)} P_{n}=0 \\
\left.\Lambda_{+++-}\right) & \partial_{z}^{2} \widehat{P}_{n}+\frac{3 z^{2}+2}{z(z+1)(z-1)} \partial_{z} \widehat{P}_{n}+\frac{n(n+2)}{(z+1)(1-z)} \widehat{P}_{n}=0 \\
\left.\Lambda_{++-+}\right) & \partial_{z}^{2} \widehat{P}_{n}+\frac{6 z^{2}-3 x-4}{z(z+1)(z-1)} \partial_{z} \widehat{P}_{n}+\frac{n z(n+5)+6}{z(z+1)(1-z)} \widehat{P}_{n}=0 \\
\left.\Lambda_{++--}\right) & \partial_{z}^{2} \widehat{P}_{n}+\frac{3 z-2}{z(z+1)(1-z)} \partial_{z} \widehat{P}_{n}+\frac{n z(n-1)-12}{4 z(z+1)(1-z)} \widehat{P}_{n}=0 \\
\left.\Lambda_{+-++}\right) & \partial_{z}^{2} \widehat{P}_{n}+\frac{6 z^{2}+3 z-4}{z(z+1)(z-1)} \partial_{z} \widehat{P}_{n}+\frac{z\left(n^{2}+5\right)-6}{z(z+1)(1-z)} \widehat{P}_{n}=0 \\
\left.\Lambda_{+-+-}\right) & \partial_{z}^{2} \widehat{P}_{n}+\frac{3 z+2}{z(z+1)(z-1)} \partial_{z} \widehat{P}_{n}+\frac{n z(n-1)+3}{z(z+1)(1-z)} \widehat{P}_{n}=0 \\
\left.\Lambda_{+--+}\right) & \partial_{z}^{2} \widehat{P}_{n}+\frac{3 z^{2}-4}{z(z+1)(z-1)} \partial_{z} \widehat{P}_{n}+\frac{n(n+2)}{(z+1)(1-z)} \widehat{P}_{n}=0 \\
\left.\Lambda_{+---}\right) & \partial_{z}^{2} \widehat{P}_{n}+\frac{3 z^{2}-2}{z(z+1)(1-z)} \partial_{z} \widehat{P}_{n}+\frac{n(4-n)}{(z+1)(z-1)} P_{n}=0 .
\end{array}
$$

The polynomial $P_{n}$ of degree $n$ exists for $\lambda_{n} \in \Lambda_{b}$ with $n$ even, that is, $\Lambda_{n}=$ $\left\{n \in \mathbb{Z}: 16 n^{2}+16 n+3\right\}$, for $\left.\Lambda_{++-+}\right)$and $\left.\Lambda_{+--+}\right)$. Therefore $E=E_{n}=\{n \in \mathbb{Z}:$ $\left.-4 n^{2}-4 n\right\}$.

The corresponding solutions for $\Lambda_{n}$ are

$$
\begin{array}{lllll}
\left.\Lambda_{+++-}\right) & \Phi_{1, n}=\widehat{P}_{2 n} \widehat{f}_{n} \Phi_{1,0}, & \Phi_{1,0}=\frac{\sqrt[4]{\left(z^{2}-1\right)^{5}}}{z} & \widehat{f}_{n}=1, & \widehat{\Psi}_{1,0}=z-\frac{1}{z} \\
\left.\Lambda_{+--+}\right) & \widehat{\Phi}_{2, n}=\widehat{Q}_{2 n} \widehat{f}_{n} \widehat{\Phi}_{2,0}, & \widehat{\Phi}_{2,0}=\frac{z^{2}}{\sqrt[4]{z^{2}-1}} & \widehat{f}_{n}=1 & \widehat{\Psi}_{2,0}=\frac{z^{2}}{\sqrt{z^{2}-1}}
\end{array}
$$

These two solutions are equivalent to the same solution of the original Schrödinger equation and correspond to the well known supersymmetric quantum mechanics approach to this Pöschl-Teller potential, 16, 17. Furthermore, for all $\lambda \in \Lambda_{n}$,

$$
\begin{aligned}
& \operatorname{DGal}\left(\widehat{\mathbf{L}}_{\lambda} / \mathbb{C}(x)\right)=\mathbb{G}^{[4]}, \quad \operatorname{DGal}\left(\widehat{L}_{\lambda} / \widehat{K}\right)=\operatorname{DGal}\left(L_{\lambda} / K\right)=e, \\
& \operatorname{dim}_{\mathbb{C}} \mathcal{E}(\widehat{\mathbf{H}}-\lambda)=2, \quad \operatorname{dim}_{\mathbb{C}} \mathcal{E}(\widehat{H}-\lambda)=\operatorname{dim}_{\mathbb{C}} \mathcal{E}(H-\lambda)=4 .
\end{aligned}
$$

\subsection{Searching for Potentials From Parameterized Differential Equa-} tions. In order to search for new potentials using $\widehat{\partial}_{z}$, our main object will be the family of differential equations presented by Darboux in [20, which can be written in the form

$$
\partial_{z}^{2} \widehat{y}+\widehat{P} \partial_{z} \widehat{y}+(\widehat{Q}-\lambda \widehat{R}) \widehat{y}=0, \quad \widehat{P}, \widehat{Q}, \widehat{R} \in \widehat{K} .
$$

We recall that some Riemann differential equations, presented previously, correspond to this kind.

When we have a differential equation in the form (6.10), we reduce it to put it in the form of the reduced algebrized Schrödinger equation $\widehat{\mathbf{H}} \Phi=\lambda \Phi$, checking that $\operatorname{Card}(\Lambda)>1$. Thus, starting with the potential $\widehat{\mathbf{V}}$ and arriving to the potential $V$, we obtain the Schrödinger equation $H \Psi=\lambda \Psi$. This heuristic methodology is detailed below. 
(1) Reduce a differential equation of the form (6.10) and put it in the form $\widehat{\mathbf{H}} \Phi=\lambda \Phi$, checking that $\operatorname{Card}(\Lambda)>1$; to avoid triviality, $\alpha$ should be a non-constant function.

(2) Write $\mathcal{W}=\frac{1}{4} \partial_{z}(\ln \alpha)$ and obtain $\widehat{V}(z)=\alpha\left(\widehat{\mathbf{V}}-\partial_{z} \mathcal{W}-\mathcal{W}^{2}\right)$

(3) Solve the differential equation $\left(\partial_{x} z\right)^{2}=\alpha$ and write $z=z(x), V(x)=$ $\widehat{V}(z(x))$.

To illustrate this method, we present the following examples.

\section{Bessel Potentials}

- (From Darboux transformations over $V=0$ ) In the differential equation

$$
\partial_{z}^{2} \Phi=\left(\frac{n(n+1)}{z^{2}}+\mu\right) \Phi, \quad \mu \in \mathbb{C}
$$

we see that $\lambda=-n(n+1)$ and $\alpha=z^{2}$. Applying the method, we obtain $\widehat{\mathbf{V}}=\mu$ and $\widehat{V}(z)=\mu z^{2}+\frac{1}{4}$ with $z=z(x)=e^{ \pm x}$. Thus, we have obtained the potentials $V(x)=\widehat{V}(z(x))=\mu e^{ \pm 2 x}+\frac{1}{4}$ (compare with [27, $\left.\S 6.9\right]$ ).

- (From Bessel differential equation) The equation

$$
\partial_{z}^{2} y+\frac{1}{z} \partial_{z} y+\frac{z^{2}-n^{2}}{z^{2}} y=0, \quad n \in \frac{1}{2}+\mathbb{Z},
$$

is transformed into the reduced equation

$$
\partial_{z}^{2} \Phi=\left(\frac{n^{2}}{z^{2}}-\frac{4 z^{2}+1}{4 z^{2}}\right) \Psi
$$

We can see that $\lambda=-n^{2}, \alpha=z^{2}$, obtaining $\widehat{\mathbf{V}}=-z^{2}-\frac{1}{4}, \widehat{V}=-z^{4}-$ $\frac{1}{4} z^{2}+\frac{1}{4}$ and $z=z(x)=e^{ \pm x}$. Thus, we have obtained the potential $V(x)=\widehat{V}(z(x))=-e^{ \pm 4 x}-\frac{1}{4} e^{ \pm 2 x}+\frac{1}{4}$ (compare with [27, $\left.\S 6.9\right]$ ).

We remark that the previous examples give us potentials related with the Morse potential, due to their solutions are given in term of Bessel functions.

We can apply this method to equations such as Whittaker, Hypergeometric and in particular, differential equations involving orthogonal polynomials (compare with [16, §5]).

\section{Final Remarks}

In this paper we gave, in contemporary terms, a formalization of original ideas and intuitions given by G. Darboux, E. Witten and L. É. Gendenshteïn in the context of the Galois theory of linear differential equations. We found the following facts.

- The superpotential is an algebraic solution of the Riccati equation associated with a potential, defined over a differential field.

- The Darboux transformation was interpreted as an isogaloisian transformation, allowing to obtain isomorphisms between their eigenrings. 
- We introduced the Hamiltonian algebrization method,which in particular allows to apply algorithmic tools such as Kovacic's algorithm to obtain the solutions, differential Galois groups and eigenring $\mathrm{s}$ of second order linear differential equations. We applied successfully this algebrization procedure to solve problems in Supersymmetric quantum mechanics.

- We can construct algebraically solvable and non-trivial algebraically quasisolvable potentials in the following ways.

(1) Giving a potential where, for $\lambda=\lambda_{0}$, the Schrödinger equation is integrable. After we put $\lambda \neq \lambda_{0}$, we check that the Schrödinger equation is integrable for more than one value of the parameter $\lambda$.

(2) Giving a superpotential to obtain the potential, after which we check whether the Schrödinger equation is integrable for more than one value of the parameter $\lambda$.

(3) Using parameterized second order linear differential equations and applying an inverse process in the Hamiltonian algebrization method. In particular, we can use algebraically solvable and algebraically quasi-solvable potentials, special functions with parameters (for example with polynomial solutions).

This paper is a starting point to analyze quantum theories through Galoisian theories. Therefore open questions and future work arise in a natural way: supersymmetric quantum mechanics with dimension greater than 2 , relationship between algebraic and analytic spectrums, etc.

As a conclusion, as happen in other areas of the field of differential equations, in view of the many families of examples studied along this paper, we can conclude that the differential Galois theory is a natural framework in which some aspects of supersymmetric quantum mechanics may appear more clearly.

\section{Acknowledgments}

The research of the first and second author has been partially supported by the MCyT-FEDER Grant MTM2006-00478. Part of this research was completed thanks to the first author's invitation in the XLIM institute in Limoges. The first author is also supported by the Sergio Arboleda university.

\section{Appendix A. Kovacic's Algorithm}

Kovacic in 1986 (see [36]) introduced an algorithm to solve the differential equation $\partial_{x}^{2} \zeta=r \zeta$, where $r \in \mathbb{C}(x)$, see also [4, 24, 25, 62. We recall this algorithm to set the notations.

Each case in Kovacic's algorithm is related with each one of the algebraic subgroups of $\mathrm{SL}(2, \mathbb{C})$ and the associated Riccatti equation

$$
\partial_{x} v=r-v^{2}=(\sqrt{r}-v)(\sqrt{r}+v), \quad v=\frac{\partial_{x} \zeta}{\zeta} .
$$

There are four cases in Kovacic's algorithm. Case 1 concerns the case when the Riccati equation has a rational solution (or more); cases 2 and 3 concern cases when the Riccati equation admits an algebraic solution; case 4 is the non-integrable case. 
Our differential equation is given by $\partial_{x}^{2} \zeta=r \zeta$ where $r=\frac{s}{t}, s, t \in \mathbb{C}[x]$. We use the following notations:

(1) $\Gamma^{\prime}$ is the set of (finite) poles of $r: \Gamma^{\prime}=\{c \in \mathbb{C}: t(c)=0\}$.

(2) $\Gamma=\Gamma^{\prime} \cup\{\infty\}$.

(3) The order $\circ\left(r_{c}\right)$ of $r$ at $c \in \Gamma^{\prime}$ is the multiplicity of $c$ as a pole of $r$.

(4) The order $\circ\left(r_{\infty}\right)$ of $r$ at $\infty$ is the order of $\infty$ as a zero of $r$, i.e $\circ\left(r_{\infty}\right)=\operatorname{deg}(t)-\operatorname{deg}(s)$.

Case 1. Let $[\sqrt{r}]_{c}$ (resp. $[\sqrt{r}]_{\infty}$ ) represent the Laurent series (if defined) of $\sqrt{r}$ at $c$ (resp. infinity). For $p \in \Gamma$, we will define $\varepsilon(p) \in\{+,-\}$ below. Finally, the complex numbers $\alpha_{c}^{+}, \alpha_{c}^{-}, \alpha_{\infty}^{+}, \alpha_{\infty}^{-}$will be defined in the first step. If the differential equation has no poles it only can fall in this case.

Step 1. For each $c \in \Gamma$ :

$\left(c_{0}\right):$ If $\circ\left(r_{c}\right)=0$, then

$$
[\sqrt{r}]_{c}=0, \quad \alpha_{c}^{ \pm}=0
$$

$\left(c_{1}\right):$ If $\circ\left(r_{c}\right)=1$, then

$$
[\sqrt{r}]_{c}=0, \quad \alpha_{c}^{ \pm}=1
$$

$\left(c_{2}\right):$ If $\circ\left(r_{c}\right)=2$, and

$$
\begin{gathered}
r=b(x-c)^{-2}+\cdots, \quad \text { then } \\
{[\sqrt{r}]_{c}=0, \quad \alpha_{c}^{ \pm}=\frac{1 \pm \sqrt{1+4 b}}{2} .}
\end{gathered}
$$

$\left(c_{3}\right):$ If $\circ\left(r_{c}\right)=2 v \geq 4$, and

$$
\begin{gathered}
r=\left(a(x-c)^{-v}+\ldots+d(x-c)^{-2}\right)^{2}+b(x-c)^{-(v+1)}+\cdots, \quad \text { then } \\
{[\sqrt{r}]_{c}=a(x-c)^{-v}+\ldots+d(x-c)^{-2}, \quad \alpha_{c}^{ \pm}=\frac{1}{2}\left( \pm \frac{b}{a}+v\right) .}
\end{gathered}
$$

$\left(\infty_{1}\right):$ If $\circ\left(r_{\infty}\right)>2$, then

$$
[\sqrt{r}]_{\infty}=0, \quad \alpha_{\infty}^{+}=0, \quad \alpha_{\infty}^{-}=1 .
$$

$\left(\infty_{2}\right)$ : If $\circ\left(r_{\infty}\right)=2$, and $r=\cdots+b x^{2}+\cdots$, then

$$
[\sqrt{r}]_{\infty}=0, \quad \alpha_{\infty}^{ \pm}=\frac{1 \pm \sqrt{1+4 b}}{2} .
$$

$\left(\infty_{3}\right)$ : If $\circ\left(r_{\infty}\right)=-2 v \leq 0$, and

$$
\begin{aligned}
r & =\left(a x^{v}+\ldots+d\right)^{2}+b x^{v-1}+\cdots, \text { then } \\
{[\sqrt{r}]_{\infty} } & =a x^{v}+\ldots+d, \quad \text { and } \quad \alpha_{\infty}^{ \pm}=\frac{1}{2}\left( \pm \frac{b}{a}-v\right) .
\end{aligned}
$$

Step 2. Find $D \neq \emptyset$ defined by

$$
D=\left\{n \in \mathbb{Z}_{+}: n=\alpha_{\infty}^{\varepsilon(\infty)}-\sum_{c \in \Gamma^{\prime}} \alpha_{c}^{\varepsilon(c)}, \forall(\varepsilon(p))_{p \in \Gamma}\right\} .
$$

If $D=\emptyset$, then we should start with the case 2 . Now, if $\operatorname{Card}(D)>0$, then for each $n \in D$ we search $\omega \in \mathbb{C}(x)$ such that

$$
\omega=\varepsilon(\infty)[\sqrt{r}]_{\infty}+\sum_{c \in \Gamma^{\prime}}\left(\varepsilon(c)[\sqrt{r}]_{c}+\alpha_{c}^{\varepsilon(c)}(x-c)^{-1}\right) .
$$


Step 3. For each $n \in D$, search for a monic polynomial $P_{n}$ of degree $n$ with

$$
\partial_{x}^{2} P_{n}+2 \omega \partial_{x} P_{n}+\left(\partial_{x} \omega+\omega^{2}-r\right) P_{n}=0 .
$$

If success is achieved then $\zeta_{1}=P_{n} e^{\int \omega}$ is a solution of the differential equation. Else, case 1 cannot hold.

\section{Case 2.}

Step 1. For each $c \in \Gamma^{\prime}$ and for $\infty$, we define the sets $E_{c} \subset \mathbb{Z}$ and $E_{\infty} \subset \mathbb{Z}$ as follows:

$\left(c_{1}\right):$ If $\circ\left(r_{c}\right)=1$, then $E_{c}=\{4\}$

$\left(c_{2}\right):$ If $\circ\left(r_{c}\right)=2$, and $r=\cdots+b(x-c)^{-2}+\cdots$, then

$$
E_{c}=\{2+k \sqrt{1+4 b}: k=0, \pm 2\} \text {. }
$$

$\left(c_{3}\right):$ If $\circ\left(r_{c}\right)=v>2$, then $E_{c}=\{v\}$

$\left(\infty_{1}\right)$ : If $\circ\left(r_{\infty}\right)>2$, then $E_{\infty}=\{0,2,4\}$

$\left(\infty_{2}\right)$ : If $\circ\left(r_{\infty}\right)=2$, and $r=\cdots+b x^{2}+\cdots$, then

$$
E_{\infty}=\{2+k \sqrt{1+4 b}: k=0, \pm 2\} .
$$

$\left(\infty_{3}\right):$ If $\circ\left(r_{\infty}\right)=v<2$, then $E_{\infty}=\{v\}$

If some $E_{c}=\emptyset$ then switch to next case.

Step 2. Compute $D$ defined by

$$
D=\left\{n \in \mathbb{Z}_{+}: \quad n=\frac{1}{2}\left(e_{\infty}-\sum_{c \in \Gamma^{\prime}} e_{c}\right), \forall e_{p} \in E_{p}, \quad p \in \Gamma\right\} .
$$

If $D=\emptyset$, then switch to case 3 . If $\operatorname{Card}(D)>0$, then for each $n \in D$, compute the corresponding rational function $\theta$ defined by

$$
\theta=\frac{1}{2} \sum_{c \in \Gamma^{\prime}} \frac{e_{c}}{x-c}
$$

Step 3. For each $n \in D$, search (via linear algebra) for a monic polynomial $P_{n}$ of degree $n$, such that

(A.2) $\partial_{x}^{3} P_{n}+3 \theta \partial_{x}^{2} P_{n}+\left(3 \partial_{x} \theta+3 \theta^{2}-4 r\right) \partial_{x} P_{n}+\left(\partial_{x} 2 \theta+3 \theta \partial_{x} \theta+\theta^{3}-4 r \theta-2 \partial_{x} r\right) P_{n}=0$.

If $P_{n}$ does not exist, then case 2 does not hold. If such a polynomial $P_{n}$ is found, set $\phi=\theta+\partial_{x} P_{n} / P_{n}$ and let $\omega$ be a solution of

$$
\omega^{2}+\phi \omega+\frac{1}{2}\left(\partial_{x} \phi+\phi^{2}-2 r\right)=0 .
$$

Then $\zeta_{1}=e^{\int \omega}$ is a solution of the differential equation.

\section{Case 3.}

Step 1. For each $c \in \Gamma^{\prime}$ and for $\infty$ we define the sets $E_{c} \subset \mathbb{Z}$ and $E_{\infty} \subset \mathbb{Z}$ as follows:

$$
\left(c_{1}\right): \text { If } \circ\left(r_{c}\right)=1 \text {, then } E_{c}=\{12\}
$$




$$
\begin{aligned}
& \left(c_{2}\right): \text { If } \circ\left(r_{c}\right)=2 \text {, and } r=b(x-c)^{-2}+\cdots, \text { then } \\
& \quad E_{c}=\{6+k \sqrt{1+4 b}: \quad k=0, \pm 1, \pm 2, \pm 3, \pm 4, \pm 5, \pm 6\} . \\
& (\infty): \text { If } \circ\left(r_{\infty}\right)=v \geq 2, \text { and } r=\cdots+b x^{2}+\cdots, \text { then } \\
& E_{\infty}=\left\{6+\frac{12 k}{m} \sqrt{1+4 b}: k=0, \pm 1, \pm 2, \pm 3, \pm 4, \pm 5, \pm 6\right\}, m \in\{4,6,12\} .
\end{aligned}
$$

Step 2. For $m \in\{4,6,12\}$ successively, compute the set $D$ defined by

$$
D=\left\{n \in \mathbb{Z}_{+}: \quad n=\frac{m}{12}\left(e_{\infty}-\sum_{c \in \Gamma^{\prime}} e_{c}\right), \forall e_{p} \in E_{p}, \quad p \in \Gamma\right\} .
$$

If $\operatorname{Card}(D)>0$, then for each $n \in D$ with its respective $m$, compute the rational function

$$
\theta=\frac{m}{12} \sum_{c \in \Gamma^{\prime}} \frac{e_{c}}{x-c}
$$

and the (denominator) polynomial $S$ defined as $S=\prod_{c \in \Gamma^{\prime}}(x-c)$.

Step 3. For each $n \in D$, with its respective $m$, compute a monic polynomial $P_{n}=P$ of degree $n$, such that its coefficients can be determined recursively by

$$
\begin{gathered}
P_{-1}=0, \quad P_{m}=-P, \\
P_{i-1}=-S \partial_{x} P_{i}-\left((m-i) \partial_{x} S-S \theta\right) P_{i}-(m-i)(i+1) S^{2} r P_{i+1},
\end{gathered}
$$

where $i \in\{0,1 \ldots, m-1, m\}$. If $P$ does not exist, then the differential equation is not integrable because it falls in Case 4.

If $P$ exists, a solution of the differential equation is given by $\zeta=e^{\int \omega}$, where $\omega$ is a zero of the polynomial of degree $m$

$$
\sum_{i=0}^{m} \frac{S^{i} P_{i}}{(m-i) !} \omega^{i}=0
$$

Case 4. In none of the above holds, then the equation is non-integrable and its Galois group is $S L(2)$.

Remark A.1 (4). If the differential equation falls only in the case 1 of Kovacic's algorithm, then its differential Galois group is given by one of the following groups:

I1: $e$ when the algorithm provides two rational solutions.

I2: $\mathbb{G}^{[n]}$ when the algorithm provides two algebraic solutions $\zeta_{1}, \zeta_{2}$ such that $\zeta_{1}^{n}, \zeta_{2}^{n} \in \mathbb{C}(x)$ and $\zeta_{1}^{n-1}, \zeta_{2}^{n-1} \notin \mathbb{C}(x)$.

I3: $\mathbb{G}^{\{n\}}$ when the algorithm provides only one algebraic solution $\zeta$ such that $\zeta^{n} \in \mathbb{C}(x)$ with $n$ minimal.

I4: $\mathbb{G}_{m}$ when the algorithm provides two non-algebraic solutions.

I5: $\mathbb{G}_{a}$ when the algorithm provides one rational solution and the second solution is not algebraic.

I6: $\mathbb{B}$ when the algorithm only provides one solution $\zeta$ such that $\zeta$ and its square are not rational functions. 


\section{References}

[1] P. B. Acosta-Humanez, Non-Autonomous Hamiltonian Systems and Morales-Ramis Theory I. The Case $\ddot{x}=f(x, t)$, SIAM Journal on Applied Dynamical Systems, 8 (2009), no. 1, 279-297

[2] P. Acosta-Humánez, M. Álvarez-Ramírez \& J. Delgado, Non-Integrability of some few body problems in two degrees of freedom, Qual. Theory Dyn. Syst. 8 (2009), no. 2, 209-239

[3] P. Acosta-Humánez \& D. Blázquez-Sanz, Hamiltonian system and variational equations with polynomial coefficients, Dynamic systems and applications, Dynamic, Atlanta, GA, Vol. 5, (2008) 6-10

[4] P. Acosta-Humánez \& D. Blázquez-Sanz, Non-integrability of some Hamiltonians with rational potentials, Discrete and Continuous Dynamical Systems Series B, 10, (2008), 265-293.

[5] G. Bachman \& L. Narici, Functional Analysis, Dover Publications (1966)

[6] M. Barkatou, Factoring Systems of Linear Functional Equations using Eigenrings, in Computer Algebra 2006, Kotsireas \& Zima Editors, World Scientific, (2007), 22-42.

[7] C. M. Bender \& S. Boettcher, Quasi-exactly solvable quartic potential, J. Phys. A: Math. Gen. 31 (1998), no. L273-L277.

[8] C. M. Bender \& G. V. Dunne, Quasi-exactly solvable systems and orthogonal polynomials, J. Math. Phys. 37 (1996), no. 1, 6-11

[9] M. Berkenbosch, Algorithms and Moduli Spaces for Differential Equations, Ph.D. thesis, University of Groningen, (2004)

[10] L. M. Berkovich \& S. A. Evlakhov, The Euler-Imshenetskii-Darboux transformation of second-order linear equations, Program. Comput. Software 32 (2006), no. 3, 154-165

[11] F. Beukers, Differential Galois theory, From number theory to physics (Les Houches, 1989), 413-439, Springer, Berlin, 1992.

[12] P. Blecua, L. J. Boya \& A. Segui, New solvable quantum-mechanical potentials by iteration of the free $V=0$ potential, Nuovo Cimento Soc. Ital. Fis. B 118 (2003), no. 6, 535-546

[13] D. Boucher, About the polynomial solutions of homogeneous linear differential equations depending on parameters. Proceedings of the 1999 International Symposium on Symbolic and Algebraic Computation (Vancouver, BC), 261-268 (electronic), ACM, New York, 1999.

[14] M. Bronstein and A. Fredet, Solving linear ordinary differential equations over $\mathbb{C}\left(x, e^{\int f(x) d x}\right)$, Proceedings of the 1999 International Symposium on Symbolic and Algebraic Computation (Vancouver, BC), (electronic), ACM, New York, (1999), 173-179.

[15] T. Chihara, An Introduction to Orthogonal polynomials, Gordon and Breach (1978)

[16] F. Cooper, A. Khare \& U. P. Sukhatme, Supersymmetry and Quantum Mechanics, Phys. Rep. 251, (1995), 267-385.

[17] F. Cooper, A. Khare \& U. P. Sukhatme, Supersymmetry in Quantum Mechanics, World Scientific Publishing Co., Inc., River Edge, NJ, 2001.

[18] T. Crespo \& Z. Hajto, Introduction to Differential Galois Theory, Monograph with an appendix by Juan J. Morales-Ruiz, Cracow University of Technology Publishers (2007)

[19] M.M. Crum, Associated Sturm-Liouville systems, Quart. J. Math. Oxford Ser. (2) 6 (1955), 121-127.

[20] G. Darboux, Sur une proposition relative aux équations linéaires, Comptes Rendus Acad. Sci. 94 (1882) 1456-1459

[21] G. Darboux, Théorie des Surfaces, II, Gauthier- Villars, 1889.

[22] J. Delsarte, Sur certaines transformations fonctionelles relatives aux équations linéaires aux déerivées partielles du second ordre, Comptes Rendus Acad. Sci. 206 (1938) 1780-1782

[23] R. Dutt, A. Khare \& U. P. Sukhatme, Supersymmetry, shape invariance and exactly solvable potentials, Am. J. Phys. 56, (1988), 163-168

[24] A. Duval and M. Loday-Richaud, Kovacic's algorithm and its application to some families of special functions, Appl. Algebra Engrg. Comm. Comput. 3 (1992), no. 3, 211-246.

[25] W. Fakler, On second order homogeneous linear differential equations with Liouvillian solutions, Theoret. Comput. Sci. 187 (1997), no. 1-2, 27-48

[26] A. Fredet, Résolution sous forme finie d'équations différentielles linéaires et extensions exponentielles, École Polytechnique, Phd Thesis (2001)

[27] A. Galindo \& P. Pascual, Quantum Mechanics I, Springer Verlag, Berlin 1990.

[28] L. Gendenshteïn, Derivation of the exact spectra of the Schrödinger equation by means of Supersymmetry, JETP Lett. 38, (1983), 356-359 
[29] J. Gibbons, A.P. Veselov, On the rational monodromy-free potentials with sextic growth, Preprint (2008), 35 pages, http://arxiv.org/abs/0807.3501

[30] E. L. Ince, Ordinary Differential Equations, Dover publications, 1927.

[31] M. Ismail, Classical and Quantum Orthogonal Polynomials in one Variable, Encyclopedia of Mathematics and its Applications 98, Cambridge University Press 1999.

[32] K. Iwasaki, H. Kimura, S. Shimomura \& M. Yoshida, From Gauss to Painlevé. A Modern Theory of Special Functions, Aspects of Mathematics E16, Friedr. Vieweg \& Sohn, Braunschweig, 1991.

[33] I. Kaplansky, An introduction to differential algebra, Hermann, 1957

[34] T. Kimura, On Riemanns Equations which are Solvable by Quadratures, Funkcialaj Ekvacioj 12 (1969), 269-281.

[35] E. Kolchin, Differential Algebra and Algebraic Groups, Academic Press, 1973.

[36] J. Kovacic, An Algorithm for Solving Second Order Linear Homogeneous Differential Equations, J. Symbolic Computation, 2, (1986), 3-43.

[37] M. G. Krein, On a continual analogue of a Christoffel formula from the theory of orthogonal polynomials, (Russian) Dokl. Akad. Nauk SSSR (N.S.) 113 (1957), 970-973.

[38] L. D. Landau \& E. M. Lifshitz, Quantum mechanics: non-relativistic theory, Pergamon Press, Oxford, 1977

[39] J. Liouville, Démonstration d'un théorème du à $M$. Sturm relatif à une classe de fonctions transcendantes, J. Math. Pures Appl. 1 (1836), 269-277

[40] J. Liouville, Mémoire sur le développement des fonctions ou parties de fonctions en séries dont les divers termes sont assujettis à satisfaire à une même équation différentielle du second ordre, contenant un paramètre variable, J. Math. Pures Appl. 1 (1836), 253-265

[41] J. Martinet \& J.P. Ramis, Thorie de Galois differentielle et resommation, Computer Algebra and Differential Equations, E. Tournier, Ed. Academic Press, London, 1989, 117-214.

[42] V. B. Matveev \& M. A. Salle, Darboux transformations and solitons, Springer Series in Nonlinear Dynamics. Springer-Verlag, Berlin, 1991.

[43] J. Morales-Ruiz, Differential Galois Theory and Non-Integrability of Hamiltonian Systems, Birkhäuser, Basel 1999.

[44] J. Morales-Ruiz \& J. P. Ramis, Galoisian obstructions to integrability of hamiltonian systems, I, Methods and Applications of Analysis 8 (2001), 33-95.

[45] G. A. Natanzon, Investigation of a one dimensional Schrödinger equation that is generated by a hypergeometric equation (Russian), Vestnik Leningrad. Univ. 1971, no. 10, 22-28

[46] A.F. Nikiforov \& V.B. Uvarov, Special Functions of Mathematical Physics, Birkhäuser, Basel (1989).

[47] J. O. Organista, M. Nowakowski \& H.C. Rosu, Shape invariance through Crum transformation, J. Math. Phys. 47 (2006), no. 12, 122104, 19 pp.

[48] E.G.C. Poole, Introduction to the theory of linear differential equations, Oxford Univ. Press, London, 1936

[49] A. Polyanin \& V. Zaitsev, Handbook of exact solutions for ordinary differential equations, CRC Press, Boca Raton, 1995.

[50] E. Prugovecki, Quantum mechanics in Hilbert space, Pure and Applied Mathematics, Vol. 41. Academic Press, New York-London, 1971

[51] J. P. Ramis, Private communication.

[52] H. Rosu, Short survey of Darboux transformations, Preprint (1999), 17 pages, http://arxiv.org/abs/quant-ph/9809056

[53] N. Saad, R. Hall \& H. Ciftci, Sextic harmonic oscillators and orthogonal polynomials, J. Phys. A 39 (2006), no. 26, 8477-8486.

[54] L. Schiff, Quantum Mechanics, McGraw-Hill, New York 1949.

[55] E. Schrödinger, The Factorization of the Hypergeometric Equation, In Proc. Roy. Irish Acad. 47 A, $53(1941)$

[56] M.F. Singer, An Outline of Differential Galois Theory, in Computer Algebra and Differential Equations, E. Tournier, ed., Academic Press, 1989, 3-58

[57] M.F. Singer, Liouvillian solutions of nth order homogeneous linear differential equations, Amer. J. Math. 103 (1981), no. 4, 661-682.

[58] M.F. Singer, Liouvillian Solutions of Linear Differential Equations with Liouvillian Coefficients, J. Symbolic Comput. 11 (1991), no. 3, 251-273. 
[59] M.F. Singer, Testing reducibility of linear differential operators: a group-theoretic perspective, Appl. Algebra Engrg. Comm. Comput. 7 (1996), no. 2, 77-104.

[60] V. Spiridonov, Universal superposition of coherent states and self-similar potentials, Physical Review A, 52 (1996), 1909-1935

[61] G. Teschl, Mathematical Methods in Quantum Mechanics With Applications to Schrödinger Operators, (2007), http://www.mat.univie.ac.at/ gerald/

[62] F. Ulmer \& J.A. Weil Note on Kovacic's algorithm. J. Symb. Comp. 22, (1996), 179-200

[63] A. V. Turbiner, Quantum mechanics: problems intermediate between exactly solvable and completely unsolvable, Soviet Phys. JETP 67 (1988), no. 2, 230-236

[64] M. Van der Put, Symbolic analysis of differential equations, Some tapas of computer algebra, Algorithms Comput. Math., 4, Springer, Berlin, (1999), 208-236,

[65] M. Van der Put \& M. Singer, Galois Theory in Linear Differential Equations, Springer Verlag, New York, (2003).

[66] M. Van Hoeij, Factorization of linear differential operators, Phd Thesis, Katholieke Universiteit Nijmegen (1995).

[67] M. Van Hoeij, Factorization of linear differential operators with rational functions coefficients, J. Symb. Comput., 24, (1997), 537-561.

[68] M. van Hoeij, Rational Solutions of the Mixed Differential Equation and its Application to Factorization of Differential Operators, in Proceedings of ISSAC96, ACM Press, (1996), 219-225

[69] M. Van Hoeij \& J.-A. Weil, An algorithm for computing invariants of differential Galois groups, J. Pure Appl. Algebra, 117\&118, (1997), 353-379

[70] M. Van Hoeij \& J.-A. Weil, Solving second order linear differential equations with Klein's theorem, in Proceedings of ISSAC'05, ACM Press, (2005), 340-347.

[71] J. A. Weil, Introduction to differential algebra and differential Galois theory, CIMPAUNESCO Lectures, Hanoi, 2001.

[72] E. Witten, Dynamical Breaking of Supersymmetry, Nucl.Phys. B 185, (1981), 513-554

(P. Acosta-Humánez) Instituto de Matemáticas y sus Aplicaciones (IMA), Universidad Sergio Arboleda, Bogotá \& Santa Marta, Colombia

E-mail address: primi@ima.usergioarboleda.edu.co

(J. Morales-Ruiz) Escuela de Caminos, Canales y Puertos, Universidad Politécnica De Madrid, Madrid, Spain

E-mail address: juan.morales-ruiz@upm.es

(J.-A. Weil) Institute XLIM (UMR CNRS n6172), DMi, Université De Limoges, LimoGES, FrANCE

E-mail address: jacques-arthur.weil@unilim.fr 